Pacific Journal of Mathematics

MAXIMAL SUBGROUPS AND AUTOMORPHISMS OF

N. BURGOYNE, ROBERT L. GRIESS, JR. AND RICHARD LYO 


\title{
MAXIMAL SUBGROUPS AND AUTOMORPHISMS OF CHEVALLEY GROUPS
}

\author{
N. Burgoyne, R. Griess ANd R. Lyons
}

\begin{abstract}
We study outer automorphisms $\alpha$ of a finite Chevalley type group $K$ and show that under certain conditions $C_{K}(\alpha)$ is a maximal subgroup of $K$.
\end{abstract}

\section{Introduction.}

(1.1) In classification problems for finite simple groups there is often the need for detailed information about known families of groups. A particular question, that can arise in proving generation lemmas, is this:

If $K$ is a known finite simple group, and $\alpha$ is an automorphism of $K$ of prime order, is $C_{K}(\alpha)$ a maximal subgroup of $K$ ?

The results in this article were motivated mainly by this question.

We consider the case when $K$ is a Chevalley type group. Simple examples show that if $\alpha$ is inner or diagonal, then, in general, $C_{K}(\alpha)$ is not maximal. However, we find that if $\alpha$ is a field or graph type automorphism then, in general, $C_{K}(\alpha)$ is maximal. There are exceptions, and we also emphasize that our results are not complete for the graph type automorphisms for the families of types $A, D, E_{6}$.

In $\S 2$ we give a general result about finite subgroups of simple algebraic groups over fields of finite characteristic: let $L$ be a finite Chevalley type group, let $G \supset L$ be a corresponding algebraic group; then, in Theorem 1 , we describe all finite groups $M$ such that $L \subseteq$ $M \subset G$. This allows us to answer the above question in a large number of cases. See 1.3 for details.

In $\S 3$, Theorem 2 gives an explicit description of all subgroups lying between $C_{K}(\alpha)$ and $K$ when $K$ is a twisted Chevalley group and $\alpha$ the automorphism induced by the usual field automorphism of the corresponding algebraic group.

In the remainder of $\S 1$ we give notation, some lemmas, and a discussion of automorphisms of Chevalley type groups.

(1.2) Notation. We use the approach of Steinberg [23] to describe the finite Chevalley type groups. We let $G$ be a simple algebraic group over the algebraically closed field $k$ of characteristic $p \neq 0$. In particular we suppose $G$ is connected and its centre $Z(G)=1$. Let $\sigma$ be an endomorphism of $G$ onto itself: thus $\sigma$ is an automorphism 
of $G$ as an abstract group and a morphism of $G$ as an algebraic group but, in general, $\sigma^{-1}$ need not be a morphism. We will be concerned almost exclusively with the case where the group

$$
G_{\sigma}=\{g \in G \mid \sigma g=g\}
$$

is finite. In this case the possibilities for $\sigma$ can be explicitly described, see $\S 11$ of [23]. Before summarizing these results we need some notation.

Let $B$ be a Borel subgroup of $G$ and $H$ a maximal torus contained in $B$. Let $\Sigma, \Sigma^{+}$and $\Pi=\left\{\alpha_{1}, \cdots, \alpha_{l}\right\}$ denote the corresponding sets of roots, positive roots, and fundamental (or simple) roots. Here $l=\operatorname{rank}$ of $G$. We use lower case Greek letters for roots (and also for endomorphisms) and reserve $\theta$ for the unique highest root in $\Sigma^{+}$ and $\theta_{s}$ for the unique highest short root in $\Sigma^{+}$(in case there are short roots). We let $\Sigma^{*}$ denote the dual root system to $\Sigma$. Let $V$ be the real vector space spanned by $\Pi$ and $(\alpha, \beta)$ the usual Euclidean inner product on $V$ and put $\langle\alpha, \beta\rangle=2(\alpha, \beta) /(\beta, \beta)$.

As usual, for each $\alpha \in \Sigma$, let $x_{\alpha}$ denote a fixed homomorphisms of $k_{+}$into $G$ satisfying $h x_{\alpha}(t) h^{-1}=x_{\alpha}(t \alpha(h))$ for $h \in H$. For convenience we often identify $H$ with $\operatorname{Hom}_{z}\left(\Gamma, k^{*}\right)$ via $h(\alpha)=\alpha(h)$ where $\Gamma$ denotes the lattice spanned by $\Sigma$ in $V$. Let $X_{\alpha}=\left\langle x_{\alpha}(t) \mid t \in k\right\rangle$; then $U=\left\langle X_{\alpha} \mid \alpha \in \Pi\right\rangle$ is the unipotent radical of $B$ and $G=\left\langle X_{\alpha} \mid \pm \alpha \in \Pi\right\rangle$.

If $N=N_{G}(H)$ then $W=N / H$ is the Weyl group. $W$ acts naturally on $V$ and if $n_{w} H=w \in W$ for some $n_{w} \in N$ we have $\left(n_{w} h n_{w}^{-1}\right)(\alpha)=$ $h\left(w^{-1} \alpha\right)$. For $\alpha \in \Sigma$ and $0 \neq t \in k$ let $n_{\alpha}(t)=x_{\alpha}(t) x_{-\alpha}\left(-t^{-1}\right) x_{\alpha}(t)$ and $n_{\alpha}=n_{\alpha}(1)$. Then $n_{\alpha}(t) \in N$ and $h_{\alpha}(t)=n_{\alpha}(t) n_{\alpha}^{-1} \in H$ and $h_{\alpha}(t)(\beta)=$ $t^{\langle\beta, \alpha\rangle}$.

The above facts are all well known and can be found, for example, in [5] and [17].

Now let $\sigma$ be an endomorphism of $G$ such that $G_{\sigma}$ is finite. By results in [23] we may suppose that $\sigma$ normalizes $B$ and $H$. Hence $\sigma$ induces a permutation on $\Pi$ which (by slight abuse of notation) we also denote by $\sigma$. From the explicit calculation in $\S 11$ of [23] we may suppose that $\sigma$ is in "standard form," i.e.,

$$
\sigma\left(x_{\alpha}(t)\right)=x_{\sigma(\alpha)}\left(t^{q} \alpha\right) \text { for } \quad \pm \alpha \in \Pi
$$

where $q_{\alpha}$ is a power of $p$. The above formula uniquely determines the action of $\sigma$ on $G$. We list the distinct possibilities for the standard form $\sigma$ in Table 1 . In column 1 we give the type of $\Sigma$; in column 2 the Dynkin diagram for $\Pi$, here " $L$ " denotes a long root; in column 3 a standard notation for $\sigma, q$ is always a positive power of $p$; in column 4 the permutation action of $\sigma$ on $\Pi$; in column 5 the values of $q_{i}=q_{\alpha_{i}}$; and in column 6 any restrictions on $l, p$ or $q$. 
TABLE 1

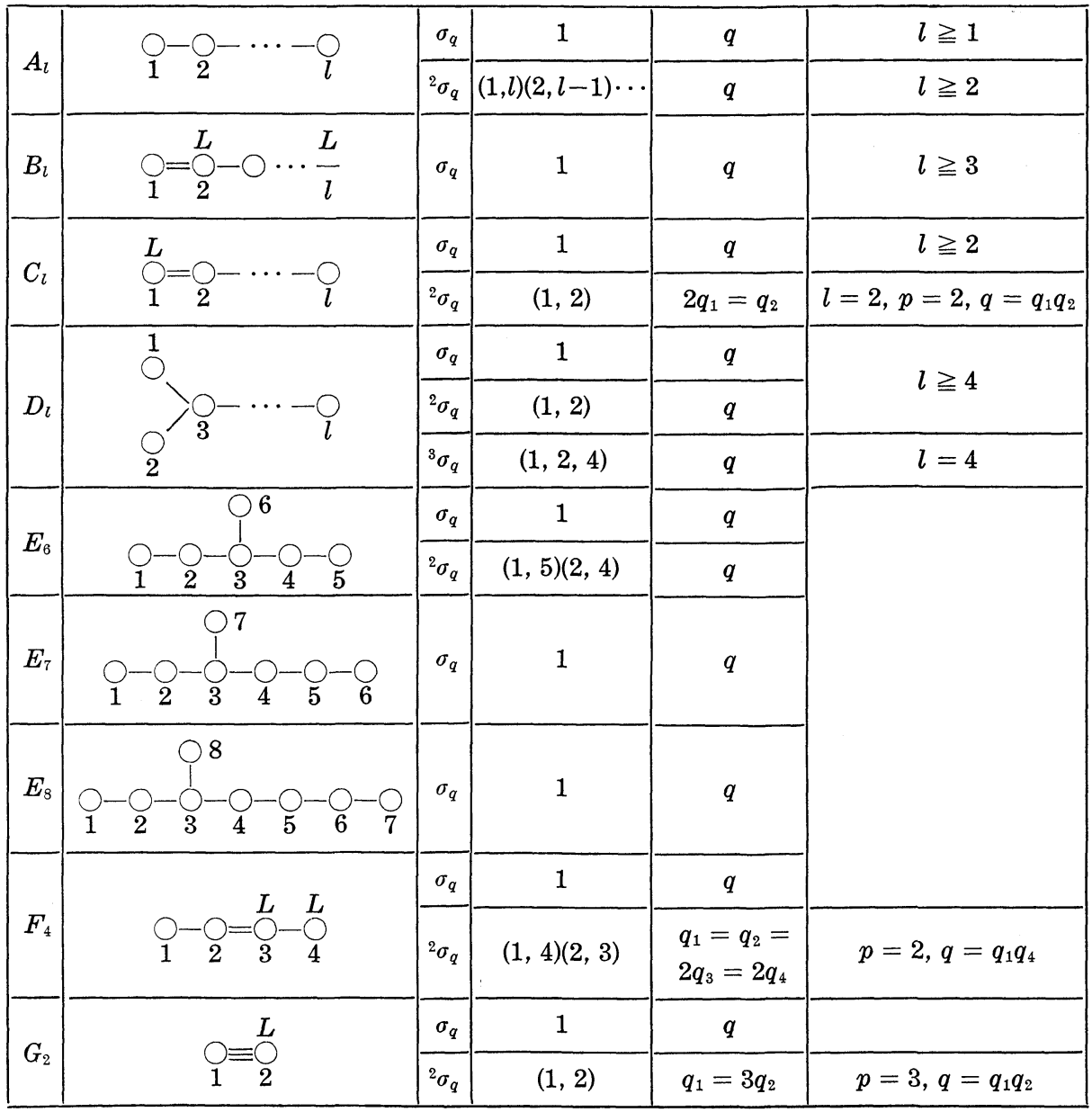

With $\sigma$ as above, if $r$ is a positive integer then $\sigma^{r}$ is also in standard form (except for $\left({ }^{3} \sigma_{q}\right)^{2}$ in the $D_{4}$ case, where the roots must be renumbered). If $\sigma=\sigma_{q}$ then $\sigma^{r}=\sigma_{q r}$. Table 2 gives the connections between $\sigma$ and $\sigma^{r}$ in the twisted cases.

TABLE 2

\begin{tabular}{|c|c|c|}
\hline Type of $G$ & $\sigma$ & $\sigma^{r}$ \\
\hline$A_{l}, D_{l}, E_{6}$ & ${ }^{2} \sigma_{q}$ & $\begin{array}{ll}\sigma_{q} r & \text { if } r=\text { even } \\
{ }^{2} \sigma_{q} r & \text { if } r=\text { odd }\end{array}$ \\
\hline$D_{4}$ & ${ }^{3} \sigma_{q}$ & $\begin{array}{ll}\sigma_{q} r & \text { if } r \equiv 0(3) \\
{ }^{3} \sigma_{q} r & \text { if } r \neq 0(3)^{(*)}\end{array}$ \\
\hline$C_{2}, F_{4} G_{2}$ & ${ }^{2} \sigma_{q}$ & $\begin{array}{l}\sigma_{q} r / 2 \text { if } r=\text { even } \\
{ }^{2} \sigma_{q} r \text { if } r=\text { odd }\end{array}$ \\
\hline
\end{tabular}

(*) but if $r \equiv-1(3), \sigma^{r}$ acts as $(1,4,2)$ on $\Pi$. 
We put $O^{p^{\prime}}\left(G_{\sigma}\right)=G_{o}^{s}$ and use the usual notation to denote these groups. With 8 exceptions, namely $A_{1}(2), A_{1}(3),{ }^{2} A_{2}(2), C_{2}(2),{ }^{2} C_{2}(2)$, ${ }^{2} F_{4}(2), G_{2}(2),{ }^{2} G_{2}(3)$, these groups are simple. Also $G_{\sigma}$ is the product of $G_{r}^{s}$ and all its diagonal automorphisms. Note that if $r \geqq 2$ then $\left|G_{\sigma}: G_{\sigma}\right|_{p}=\left|G_{o}^{s}: G_{\sigma}^{s}\right|_{p} \neq 1$.

Keeping the above notation we give two elementary lemmas.

LEMMA 1.1. $N_{G}\left(U_{\sigma}\right) \subseteq B$.

Proof. If $g \in N_{G}\left(U_{o}\right)$ then using the Bruhat normal form $g=$ $b n_{w} u$. Now $U_{o}^{b n_{w}}=U_{o}^{u^{-1}} \subseteq U$ and also $U_{a}^{b} \subseteq U$. For each $i=1, \cdots, l$ an $x_{\alpha_{i}}(t)$ with $t \neq 0$ occurs in some element of $U_{\sigma}$. Now $x_{\alpha_{i}}(t)^{b}=$ $x_{\alpha_{i}}\left(t^{\prime}\right) v$ where $t^{\prime} \neq 0$ and only $x_{\beta}$ with $\beta$ of height $\geqq 2$ occur in $v$. Hence $w\left(\alpha_{i}\right) \in \Sigma^{+}$all $i$. Hence $w=1$ and so $g \in B$.

Lemma 1.2. Let $K$ be a group, $G_{o}^{s} \subseteq K \leqq G_{o}$. Then $C_{G}(K)=1$ and $N_{G}(K)=G_{\sigma}$.

Proof. Let $g \in C_{G}(K)$. By the above lemma, $g \in B$. Now $\left[g, N_{\sigma}\right]=$ 1 implies $g \in H$ and identifying $H$ with $\operatorname{Hom}\left(\Gamma, k^{*}\right)$ gives $g\left(\alpha_{i}\right)=1$ for $i=1, \cdots, l$ and so $g=1$.

Next let $g \in N_{G}(K)$; then for all $k \in K, g^{-1} k g=\sigma\left(g^{-1} k g\right)$. Thus $g \sigma\left(g^{-1}\right) \in C_{G}(K)=1$ and so $g \in G_{o}$. Since $G_{o} / G_{o}^{s}$ is abelian we have $N_{G}(K)=G_{\sigma}$.

Finally we mention that our notation from finite group theory is standard, see for example [13]. In particular we use $g^{x}=x^{-1} g x$.

(1.3) Automorphisms of $G_{\sigma}$. Let $G$ and $\sigma$ be as in (1.2). In

TABLE 3

\begin{tabular}{|c|c|c|c|c|}
\hline & $G$ & $\sigma\left(q=p^{f}\right)$ & Coset representatives & $\operatorname{Aut}\left(G_{\sigma}\right) / \operatorname{Inn}\left(G_{o}\right)$ \\
\hline \multirow{2}{*}{$\begin{array}{l}A_{l} \\
D_{l} \\
E_{6}\end{array}$} & \multirow{2}{*}{$\begin{array}{l}l \geqq 2 \\
l \geqq 5\end{array}$} & $\sigma_{q}$ & \multirow{2}{*}{$\sigma_{p}{ }^{i},{ }^{2} \sigma_{p} i \quad 1 \leqq i \leqq f$} & $Z_{2} \times Z_{f}$ \\
\hline & & ${ }^{2} \sigma_{q}$ & & $Z_{2 f}$ \\
\hline \multirow{3}{*}{\multicolumn{2}{|c|}{$D_{4}$}} & $\sigma_{q}$ & $\sigma_{p^{i}}, \sigma_{p^{i}},{ }^{3} \sigma_{p^{i}} 1 \leqq i \leqq f$ & $S_{3} \times Z_{f}$ \\
\hline & & ${ }^{2} \sigma_{q}$ & $\sigma_{p}{ }^{i},{ }^{2} \sigma_{p^{i}} \quad 1 \leqq i \leqq f$ & $Z_{2 f}$ \\
\hline & & ${ }^{3} \sigma_{q}$ & $\sigma_{p}{ }^{i},{ }^{3} \sigma_{p} i \quad 1 \leqq i \leqq f$ & $Z_{3 f}$ \\
\hline & \multirow{2}{*}{$\begin{array}{l}p=2 \\
p=2 \\
p=3\end{array}$} & $\sigma_{q}$ & $\sigma_{p^{i}},{ }^{2} \sigma_{p} i-1 \quad 1 \leqq i \leqq f$ & $Z_{2 f}$ \\
\hline & & ${ }^{2} \sigma_{q}$ & ${ }^{2} \sigma_{p} i-1 \quad 1 \leqq i \leqq f$ & $Z_{f}$ \\
\hline \multicolumn{2}{|c|}{ All others } & $\sigma_{q}$ & $\sigma_{p^{i}} \quad 1 \leqq i \leqq f$ & $Z_{f}$ \\
\hline
\end{tabular}


particular we suppose $\sigma$ is in the standard form given in Table 1 for a fixed choice of $B, H$ and $x_{\alpha}$ 's in $G$. Hence $G_{\sigma}$ is finite.

Let $\lambda$ be any endomorphism of $G$ satisfying $\lambda \sigma=\sigma \lambda$, then $\lambda$ induces an element $\bar{\lambda} \in \operatorname{Aut}\left(G_{\sigma}\right)$. The structure of Aut $\left(G_{\sigma}\right) / \operatorname{Inn}\left(G_{\sigma}\right)$ is described in [5]. Using these results it is straightforward to check that the endomorphisms $\lambda$ listed in Table 3 give, via $\bar{\lambda}$, a complete set of coset representatives for $\operatorname{Inn}\left(G_{\sigma}\right)$ in Aut $\left(G_{\sigma}\right)$. Note that $G_{\sigma}$ is not, in general, simple.

Now suppose $\bar{\lambda}$ is one of the "coset representatives" given above and let $\alpha$ be any element in the coset $\operatorname{Inn}\left(G_{\sigma}\right) \bar{\lambda}$. Thus $\alpha=i_{g} \bar{\lambda}$ where $i_{g}(x)=g x g^{-1}$ for $g, x \in G_{g}$.

Lemma 1.3. Let $\lambda, \alpha=i_{g} \bar{\lambda}$ be as above. Suppose $\bar{\lambda}$ and $\alpha$ both have order $r$ and $\lambda^{r}=\sigma$. Then $\bar{\lambda}$ and $\alpha$ are conjugate under $\operatorname{Inn}\left(G_{\sigma}\right)$.

Proof. Using $\bar{\lambda} i_{g}=i_{\lambda(g)} \bar{\lambda}$, and $Z\left(G_{o}\right)=1, \alpha^{r}=\bar{\lambda}^{r}=1$ gives $g \lambda(g) \cdots$ $\lambda^{r-1}(g)=1$. By Lang's theorem [20] there exists $k \in G$ such that $g=k^{-1} \lambda(k)$. Hence $k=\lambda^{r}(k)=\sigma(k)$ and so $k \in G_{\sigma}$ and $\alpha=i_{k}^{-1} \bar{\lambda} i_{k}$.

Lemma 1.4. Let $\bar{\lambda}, \alpha=i_{g} \bar{\lambda}$ be as above. Suppose $\bar{\lambda}, \alpha$ both have order $r$. Suppose $\lambda^{r} \neq \sigma$ but that $\lambda_{1}^{r}=\sigma$ for some $\lambda_{1}$ such that $\left\langle\bar{\lambda}_{1}\right\rangle=\langle\bar{\lambda}\rangle$. Then $\bar{\lambda}$ and $\alpha$ are conjugate under $\operatorname{Inn}\left(G_{\sigma}\right)$.

Proof. Suppose $\bar{\lambda}_{1}=\bar{\lambda}^{m}$ for some integer $m$. Let $\beta=\alpha^{m}$ then $\beta=i_{k} \bar{\lambda}_{1}$ for some $k \in G_{\sigma}$. Since $\bar{\lambda}_{1}$ and $\beta$ both have order $r$, Lemma 1.3 implies that $\bar{\lambda}_{1}$ and $\beta$ are conjugate under Inn $\left(G_{\sigma}\right)$. Suppose $\bar{\lambda}=\bar{\lambda}_{1}^{d}$ for some integer $d$ then, since $\bar{\lambda}$ and $\alpha$ have the same order, we have $\alpha=\beta^{d}$. Hence $\bar{\lambda}$ and $\alpha$ are conjugate under $\operatorname{Inn}\left(G_{\sigma}\right)$.

Using these two results an inspection of Table 3 immediately yields

Proposition 1.1. Let $\lambda$ be as above and suppose $\bar{\lambda}^{r}=1$, where $r$ is a prime number. Then, apart from the possible exceptions (i), (ii) given below, the coset Inn $\left(G_{o}\right) \bar{\lambda}$ contains a unique class of elements of order $r$, under conjugation by $\operatorname{Inn}\left(G_{\sigma}\right)$, and furthermore there exists an endomorphism $\lambda_{1}$ such that $\lambda_{1}^{r}=\sigma$ and $\left\langle\bar{\lambda}_{1}\right\rangle=\langle\bar{\lambda}\rangle$. The possible exceptions are:

(i ) $\quad G=A_{l}(l \geqq 2), D_{l}(l \geqq 4), E_{6}$ with $\left\{\begin{array}{l}\sigma=\sigma_{q} \text { with } \lambda={ }^{2} \sigma_{q} \\ \sigma={ }^{2} \sigma_{q} \text { with } \lambda=\sigma_{q} .\end{array}\right.$

$$
G=D_{4} \text { with }\left\{\begin{array}{l}
\sigma=\sigma_{q} \text { with } \lambda={ }^{3} \sigma_{q} \\
\sigma={ }^{3} \sigma_{q} \text { with } \lambda=\sigma_{q}
\end{array}\right.
$$


Note that $r=2$ in (i) and $r=3$ in (ii). These exceptions do occur; in fact only for $G=A_{l}$ with $l=$ even is there a single class for the given $\lambda$. For $G=D_{l}$ the number of classes increases as $l / 2$.

We now consider when $C=C_{G_{\sigma}^{s}}(\alpha)$ is a maximal sugroup of $G_{\sigma}^{s}$. Apart from the exceptions (i), (ii) Proposition 1.1 implies first that we may suppose $\alpha=\bar{\lambda}$, and next, since $C_{G_{\sigma}^{s}}(\bar{\lambda})=C_{G_{\sigma}^{s}}\left(\bar{\lambda}_{1}\right)$, we may suppose that $\lambda^{r}=\sigma$. Now an immediate consequence of Theorem 1 is that, if $C$ is nonsolvable, then it is always maximal in $G_{o}^{s}$.

In the exceptions (i), (ii) we have a more complicated problem, especially when $r=p$. Theorem 2 is one step towards a solution.

\section{Theorem 1 .}

(2.1) Statement of results. Let $G$ be a simple algebraic group over an algebraically closed field $k$ of characteristic $p \neq 0$. Let $\lambda$ be an endomorphism of $G$ onto itself such that the subgroup $G_{\lambda}$ of fixed points is finite. As discussed in (1.2) we may suppose $\lambda$ is in standard form. If $r$ is any positive integer the endomorphism $\lambda^{r}$ is also in standard form. The possibilities for $\lambda$ and the corresponding $\lambda^{r}$ are listed in the tables in $\S 1$.

Recall that $G_{\lambda}^{s}=O^{p^{\prime}}\left(G_{\lambda}\right)$ and, with eight exceptions, is a simple group. $G_{\lambda}$ is the product of $G_{\lambda}^{s}$ and all its diagonal-type outer automorphisms.

If $G, \lambda$ are such that $G_{\lambda}^{s}$ is one of the three groups $A_{1}(2), A_{1}(3)$, ${ }^{2} C_{2}(2)$ we call this an exceptional case.

THEOREM 1. Let $G, \lambda$ be as above and not an exceptional case. Let $M$ be a finite subgroups of $G$ containing $G_{\lambda}^{s}$. Then there exists a positive integer $r$ such that (with $\mu=\lambda^{r}$ )

$$
G_{\mu}^{s} \subseteq M \leqq G_{\mu} \text {. }
$$

An immediate consequence is that if $G, \lambda$ are as in the statement of the theorem and $\mu=\lambda^{r}$ where $r$ is a prime number then $G_{\lambda} \cap G_{\mu}^{s}$ is a proper maximal subgroup of $G_{\mu}^{s}$.

The proof of the theorem is given in (2.3)-(2.5). It was necessary to handle the case $G_{\lambda}={ }^{2} G_{2}(q)$ separately and this occupies (2.5). In the general case the proof falls into two parts. In (2.3) we first describe $N_{G}\left(U_{\lambda}\right)$ (see Lemma 2.3) then use this to show there exists a (unique) integer $r$ such that, if $\mu=\lambda^{r}, U_{\mu} \in \operatorname{Syl}_{p}(M)$. In (2.4) we combine this result with induction on the rank of $G$ and show that either (a) the theorem holds, or (b) $M$ contains a proper strongly 2-embedded subgroup. Using results of $\mathrm{H}$. Bender [2] we easily rule out (b). 
(2.2) The exceptional cases. If $G, \lambda$ are an exceptional case there do exist finite subgroups $M$ such that $G_{\lambda}^{s} \subset M \subset G$ and which do not satisfy the conclusion of the theorem. We now describe all these 'exceptional' $M$.

If $G_{\lambda}^{s}=A_{1}(2)$ or $A_{1}(3)$ we use results of Dickson, see [6]. If $G_{\lambda}^{s}={ }^{2} C_{2}(2)$ we use Suzuki [25] and the recent work of Flesner [11].

$A_{1}(2): \quad M$ is a subgroup of a dihedral group of order $2(q \pm 1)$ in $G_{\lambda^{r}}=A_{1}(q)$ where $q=2^{r}$ and $q \pm 1 \equiv 0(\bmod 3)$.

$A_{1}(3): \quad M$ is a subgroup of $G_{\lambda^{2}}^{s}=A_{1}(9)$ and is isomorphic to the alternating group on 5 letters.

${ }^{2} C_{2}(2): \quad M$ is either a subgroup of a group of order $4(q \pm \sqrt{2 q}+1)$ in $G_{\lambda^{r}}={ }^{2} C_{2}(q)$ where $q=2^{r}$ and $r$ is odd, or else $M$ is a subgroup of $G_{\lambda^{2 r}}=C_{2}\left(2^{r}\right)$ and is isomorphic to a subgroup of the four dimensional orthogonal group of index one over $\boldsymbol{F}_{2^{r}}$.

(2.3) Proof. First part. We assume throughout this subsection that $G, \lambda$ satisfy the hypothesis of the theorem and also that $G_{\lambda} \neq$ ${ }^{2} G_{2}(q)$. The main technique in proving the following lemmas is the Chevalley commutator relations together with the known embedding of $U_{\lambda}$ in $U$.

The subgroups $B, U, H$ and sets of roots $\Sigma, \Pi$, etc. are as described in (1.2).

LEMMA 2.1. $C_{U}\left(U_{\lambda}\right)=Z(U)$.

Proof. We call two roots $\rho, \sigma \in \Sigma$ fundamentally independent if $\rho+\sigma \in \Sigma$ and $\{\rho, \sigma\}$ is a fundamental system in the rank 2 system $(\boldsymbol{Z} \rho+\boldsymbol{Z} \sigma) \cap \Sigma$. If $\rho$ and $\sigma$ are fundamentally independent, then in $G$ we have a commutator relation $\left[x_{\rho}(t), x_{\sigma}(u)\right]=x_{\rho_{+}}( \pm t u) \cdots$. Note that $\rho, \sigma \in \Sigma$ and $(\rho, \sigma)<0$, then $\rho$ and $\sigma$ are fundamentally independent unless $\Sigma=G_{2}$ and $\rho$ and $\sigma$ are short roots inclined at $120^{\circ}$.

Recall that $\theta$ is the highest root in $\Sigma^{+}$, and $\theta_{s}$ is the highest short root (in the case of two root lengths). Let $D=\{x \in R \Sigma \mid(x, \sigma) \geqq 0$ for all $\left.\sigma \in \Sigma^{+}\right\}$be the usual fundamental domain for the action of $W$ on $\boldsymbol{R} \Sigma$. Since $W$ is transitive on roots of a given length, $D$ contains exactly one root of each length. Clearly $\theta \in D$; otherwise for some $\sigma \in \Sigma^{+}$, we would have $(\theta, \sigma)<0$ and so $\theta+\sigma \in \Sigma$. Since $D$ is also a fundamental domain for the dual root system $\Sigma^{*}, D$ contains the highest root of $\Sigma^{*}$, whose dual-which is $\theta_{s}$-therefore lies in $D$. Thus, for any $\rho \in \Sigma-\left\{\theta, \theta_{s}\right\}$, there is $\sigma \in \Sigma^{+}$such that $(\rho, \sigma)<0$.

Hence:

$\left(^{*}\right)$ If $\rho \in \Sigma^{+}-\left\{\theta, \theta_{s}\right\}$, then there exist $\sigma \in \Sigma^{+}$such that $\rho$ and 
$\sigma$ are fundamentally independent, unless $\Sigma=G_{2}$ and $\rho$ is the sum of the fundamental roots.

We also need:

$\left.{ }^{* *}\right)$ Suppose $\Sigma$ has two root lengths, $\rho \in \Sigma^{+}$, and $\theta_{s}<\rho<\theta$. Then $\theta_{s}+\rho \notin \Sigma$, and there exists $\sigma \in \Sigma^{+}$such that $\rho$ and $\sigma$ are fundamentally independent and $\theta_{s}+\sigma \notin \Sigma$.

To prove this, note that if $\sigma$ is any long root in $\Sigma^{+}$, then $\theta_{s}+$ $\sigma \notin \Sigma$, since otherwise $\theta_{s}+\sigma$ would be a short root. In particular, $\theta_{s}+\rho \notin \Sigma$ since $\rho\left(>\theta_{s}\right)$ is long. Now, using $\left(^{*}\right)$, choose $\sigma \in \Sigma^{+}$such that $\rho$ and $\sigma$ are fundamentally independent. Since $\rho+\sigma\left(>\theta_{s}\right)$ is long, $\sigma$ is long, so $\theta_{s}+\sigma \notin \Sigma$, as required.

For any $u \in U$, we have $u=\Pi_{p \in \Sigma^{+}} x_{\rho}\left(t_{\rho}\right), t_{\rho} \in k$. We take all products over $\Sigma^{+}$to be in increasing order with respect to $\Sigma^{+}$. We set $\operatorname{supp}(u)=\left\{\rho \in \Sigma^{+} \mid t_{\rho} \neq 0\right\}$ for $u \in U$.

Now consider the case $\lambda=\sigma_{q}$, where $q$ is some power of $p$, so $U_{\lambda}=\left\{\Pi_{\rho} x_{\rho}\left(t_{\rho}\right) \mid t_{\rho} \in G F(q)\right\}$. Let $u \in C_{U}\left(U_{\lambda}\right)$. We shall show $\operatorname{supp}(u) \subseteq$ $\left\{\theta_{s}, \theta\right\}$. Let $\rho_{0}$ be the least element of $\operatorname{supp}(u)$, so

$$
u=x_{\rho_{0}}\left(t_{\rho_{0}}\right) \prod_{\rho>\rho_{0}} x_{\rho}\left(t_{\rho}\right), t_{\rho_{0}} \neq 0 \text {. }
$$

If there exists $\sigma \in \Sigma^{+}$such that $\rho_{0}$ and $\sigma$ are fundamentally independent, then we get $1=\left[u, x_{\sigma}(1)\right]=x_{\rho_{0}+\sigma}\left( \pm t_{\rho_{0}}\right) \cdots$, contradiction. Thus no such $\sigma$ is available. By $\left(^{*}\right)$, either $\rho_{0} \in\left\{\theta_{s}, \theta\right\}$, or $\Sigma=G_{2}$ and $\rho_{0}=$ $\alpha+\beta$, where $\Pi=\{\alpha, \beta\}$, with, say, $\alpha$ long and $\beta$ short. In this last case, $1=\left[u, x_{\alpha+2 \beta}(1)\right]=x_{2 \alpha+3 \beta}\left( \pm 3 t_{\rho_{0}}\right)$ and $1=\left[u, x_{\beta}(1)\right]=x_{\alpha+2 \beta}\left( \pm 2 t_{\rho_{0}}\right)$, so $3 t_{\rho_{0}}=2 t_{\rho_{0}}=0$, contradiction. Hence, $\rho_{0} \in\left\{\theta_{s}, \theta\right\}$. Suppose $\rho_{0}=\theta_{s}$ and let $\rho_{1}$ be the least element of $\operatorname{supp}(u)$ greater than $\rho_{0}$ (if $\operatorname{supp}(u) \neq$ $\left.\left\{\rho_{0}\right\}\right)$. If $\rho_{1} \neq \theta$, choose $\sigma$ so that $\rho_{1}$ and $\sigma$ are fundamentally independent and $\rho_{0}+\sigma \notin \Sigma$ (by (**)). Then $1=\left[u, x_{\sigma}(1)\right]=x_{\rho_{1}+\sigma}\left( \pm t_{\rho_{1}}\right) \cdots$ contradicting $t_{\rho_{1}} \neq 0$. Therefore $\rho_{1}=\theta$, so $\operatorname{supp}(u) \cong\left\{\theta_{s}, \theta\right\}$. If actually $\operatorname{supp}(u) \subseteq\{\theta\}$ for all $u \in C_{U}\left(U_{\lambda}\right)$, then $C_{U}\left(U_{\lambda}\right) \subseteq X_{\theta} \subseteq Z(U)$, as required. So we may assume $\theta_{s} \in \operatorname{supp}(u)$, i.e., $u=x_{\theta_{s}}(t) x_{\theta}\left(t^{\prime}\right)$ with $t \neq 0$. There exist a (short) $\sigma \in \Sigma^{+}$such that $\theta_{s}+\sigma \in \Sigma$. We get $1=\left[u, x_{o}(1)\right]=x_{\theta_{s}}( \pm m t) \cdots$, where $m=2$ if $G$ is of type $B, C$ or $F_{4}$ and $m=3$ if of type $G_{2}$. Hence $m=p$ and in precisely these case $Z(U)=X_{\theta_{s}} X_{\theta} \supseteqq C_{U}\left(U_{\lambda}\right)$, as required.

Next, suppose $\Sigma$ has one root length, $\lambda={ }^{2} \sigma_{q}$ or ${ }^{3} \sigma_{q}$, and $\Sigma \neq A_{2 n}$. Let $u \in C_{U}\left(U_{\lambda}\right)$, let $\rho_{0}$ be the least element of $\operatorname{supp}(u)$, so

$$
u=x_{\rho_{0}}\left(t_{\rho_{0}}\right) \prod_{\rho>\rho_{0}} x_{\rho}\left(t_{\rho}\right)
$$

with $t_{\rho_{0}} \neq 0$. Suppose $\rho_{0} \neq \theta$, and choose $\sigma \in \Sigma^{+}$such that $\sigma$ and $\rho_{0}$ are fundamentally independent. Let $\bar{x}_{\sigma}$ be the product of the distinct images of $x_{\sigma}(1)$ under the powers of $\lambda$, so that $\bar{x}_{\sigma} \in U_{\lambda}$ and $\bar{x}_{\sigma}=x_{\sigma}(1) x_{\lambda(\sigma)}(1) \cdots$. The roots $s, \lambda(s), \cdots$ have the same height, so $1=$ 
$\left[u, \bar{x}_{o}\right]=x_{\rho_{0}+o}\left( \pm t_{\rho_{0}}\right) \cdots$, contradiction. Thus $\rho_{0}=\theta$, so $u \in X_{\theta} \subseteq Z(U)$.

If $\Sigma=A_{2 n}$ and $\lambda={ }^{2} \sigma_{q}$, essentially the same argument works, except that if $\sigma+\lambda(\sigma) \in \Sigma$, we define $\bar{x}_{\sigma}=x_{\sigma}(1) x_{\lambda(\sigma)}(1) x_{\sigma+\lambda(\sigma)}(b)$, with $b \in G F\left(q^{2}\right)$ chosen to satsfy $b+b^{q}=1$; if $\sigma=\lambda(\sigma)$, we define $\bar{x}_{\sigma}=x_{\sigma}(b)$ with $b$ chosen to satisfy $b+b^{q}=0$. Then $1=\left[u, \bar{x}_{\sigma}\right]=x_{\rho_{0}+o}\left( \pm t_{\rho_{0}}\right) \cdots$ or $x_{\rho_{0}+\sigma}\left( \pm b t_{\rho_{0}}\right) \cdots$, contradiction, unless $\rho_{0}=\theta$.

Suppose $\Sigma=C_{2}$ and $\lambda={ }^{2} \sigma_{q}$. Then $q=2 n^{2}, n=2^{f}>1$, by assumption. Let $\Pi=\{\alpha, \beta\}$, with $\alpha$ long. For every $t \in G F(q)$, let $\bar{x}(t)=x_{\alpha}(t) x_{\beta}\left(t^{n}\right) x_{\alpha+\beta}\left(t^{1+n}\right) \in U_{\lambda}$. Suppose $u=\Pi_{\rho} x_{\rho}\left(t_{\rho}\right) \in C_{U}\left(U_{\lambda}\right)$. Then $1=[u, \bar{x}(t)]=x_{\alpha+\beta}\left(t t_{\beta}+t^{n} t_{\alpha}\right) x_{\alpha+2 \beta}\left(t t_{\beta}^{2}+t^{2 n} t_{\alpha}\right)$ for all $t \in G F(q)$. Hence $t t_{\beta}+t^{n} t_{\alpha}=t t_{\beta}^{2}+t^{2 n} t_{\alpha}=0$. With $t=1$, we conclude $t_{\alpha}=t_{\beta}=t_{\beta}^{2}$. Now if $t_{\alpha}=t_{\beta}=1$, we get $t^{n}=t^{2 n}$ for all $t \in G F(q)$, so $q=2$, contradiction. Hence $t_{\alpha}=t_{\beta}=0$, so $u \in X_{\alpha+\beta} X_{\alpha+2 \beta} \in Z(U)$.

Suppose $\Sigma=F_{4}$ and $\lambda={ }^{2} \sigma_{q}$. We need:

$\left({ }^{* *}\right)$ if $\rho_{0} \in \Sigma^{+}-\left\{\theta_{s}, \theta\right\}$, then there exist $\sigma, \sigma^{\prime} \in \Sigma^{+}$and an element $\bar{x}_{\sigma}=x_{\sigma}(1) x_{\sigma^{\prime}}(1) \prod_{\rho} x_{\rho}\left(t_{\rho}\right)$ of $U_{\lambda}$ such that (i) $h t(\sigma)=h t\left(\sigma^{\prime}\right)$, and $t_{\rho}=0$ unless $h t(\rho)>h t(\sigma)$, (ii) $\rho_{0}$ and $\sigma$ are fundamentally independent, and $\rho_{0}+\sigma-\sigma^{\prime} \notin \Sigma$.

Assuming this, let $u \in C_{U}\left(U_{\lambda}\right)$ and let $\rho_{0}$ be the least element of $\operatorname{supp}(u), u=x_{\rho_{0}}\left(t_{\rho_{0}}\right) \cdots$. If $\rho_{0} \neq \theta_{s}$ or $\theta$, choose $\sigma, \sigma^{\prime}$, and $\bar{x}_{\sigma}$ as in $\left({ }^{* *}\right)$. Then $1=\left[u, \bar{x}_{\sigma}\right]=x_{\rho_{0}+\sigma}\left(t_{\rho_{0}}\right) \cdots$ because the condition $\rho_{0}+\sigma-$ $\sigma^{\prime} \notin \Sigma$ guarantees that the only way to express $\rho_{0}+\sigma$ as the sum of an element of $\operatorname{supp}(u)$ and an element of $\operatorname{supp}\left(\bar{x}_{\sigma}\right)$ is as $\rho_{0}+\sigma$. But $t_{\rho_{0}} \neq 0$, so $\rho_{0} \in\left\{\theta_{s}, \theta\right\}$. Hence $\theta_{s}$ is the only possible short root in $\operatorname{supp}(u)$. Since $\lambda(u) \in C_{U}\left(U_{2}\right)$, and $\lambda\left(\theta_{s}\right)=\theta$, the same argument applied to $\lambda(u)$ implies that the only possible long root in supp $(u)$ is $\theta$. Hence $u \in X_{\theta_{s}} X_{\theta}=Z(U)$, and we are done.

To prove $\left(^{* * *}\right.$ ) we examine $\Sigma$ in detail. Let $\Pi=\left\{\alpha_{1}, \alpha_{2}, \alpha_{3}, \alpha_{4}\right\}$, read from one end of the Dynkin diagram to the other, with $\alpha_{1}$ short. We write the root $\sum_{i=1}^{4} n_{i} \alpha_{i}$ as $n_{1} n_{2} n_{3} n_{4}$. Thus $\theta_{s}=2321$ and $\theta=2432$. If $\rho_{0} \in\{0100,0110,0221,1221,1321\}$, take $\sigma=1000, \sigma^{\prime}=0001, \bar{x}_{\sigma}=x_{\sigma}(1) x_{\sigma^{\prime}}(1)$. If $\rho_{0} \in\{0010,0210,2431\}$, take $\sigma=0001, \sigma^{\prime}=1000, \bar{x}_{\sigma}=x_{\sigma}(1) x_{\sigma^{\prime}}(1)$. In the remaining cases, take $\bar{x}_{\sigma}=x_{\sigma}(1) x_{\sigma^{\prime}}(1) x_{\sigma+\sigma^{\prime}}(1)$. If $\rho_{0} \in\{1000,0011$, $1110,1111,2221\}$, take $\sigma=0100, \sigma^{\prime}=0010$. If $\rho_{0} \in\{0001,1100,0211$, $1211,2211\}$, take $\sigma=0010, \sigma^{\prime}=0100$. If $\rho_{0} \in\{1210,2210,2421\}$, take $\sigma=0011, \sigma^{\prime}=1100$. If $\rho_{0}=0111$, take $\sigma=1100, \sigma^{\prime}=0011$. Then $\left({ }^{* *}\right)$ is easily verified.

\section{LemMA 2.2. $C_{G}\left(U_{\lambda}\right)=Z(U)$.}

Proof. By Lemma 1.1, $C_{G}\left(U_{\lambda}\right) \cong B$, so by Lemma 2.1, it suffices to show $C_{B}\left(U_{\lambda}\right) \subseteq U$. Let $U^{\prime}=\left\langle X_{\rho} \mid \rho \in \Sigma^{+}-\Pi\right\rangle$, define $\bar{B}=B / U^{\prime}$, and for any $A \subseteq B$ write $\bar{A}$ for $A U^{\prime} / U^{\prime}$. It suffices to show $C_{\bar{B}}\left(\bar{U}_{\lambda}\right) \subseteq \bar{U}$. Now $\bar{U}$ is the direct product of $\bar{X}_{\rho}$ over all $\rho \in \Pi$, and $\bar{X}_{\rho} \cong X_{\rho}$ for 
$\rho \in \Pi$. In particular $\bar{U}$ is abelian, so $C_{\bar{B}}\left(\bar{U}_{\lambda}\right)=\bar{U} C_{\bar{H}}\left(\bar{U}_{\lambda}\right)$, as $\bar{B}=\bar{U} \bar{H}$. Thus it suffices to show $C_{\bar{H}}\left(\bar{U}_{\lambda}\right)=1$. Suppose $h \in H$ and $\bar{h} \in C_{\bar{H}}\left(\bar{U}_{\lambda}\right)$. For any $\rho \in \Pi$, there exists $u \in U_{\lambda}$ such that $\rho \in \operatorname{supp}(u)$, say $u=$ $x_{\rho}\left(t_{\rho}\right) \cdots, t_{\rho} \neq 0$. Then, identifying $H$ with $\operatorname{Hom}\left(\Gamma, k^{*}\right), \overline{1}=[\bar{h}, \bar{u}]=$ $\overline{x_{\rho}\left(t_{\rho}(h(\rho)-1)\right)} \cdots$, so $h(\rho)=1$. Thus $h=1$, as required.

Lemma 2.3. $N_{G}\left(U_{\lambda}\right)=\left\langle B_{\lambda}, Z(U)\right\rangle$.

Proof. Let $g \in N_{G}\left(U_{\lambda}\right)$. Then $g^{-1} \lambda(g) \in C_{G}\left(U_{\lambda}\right)$. By Lemma 2.2, $g^{-1} \lambda(g) \in Z(U)$. Since $Z(U)\left(=X_{\theta}\right.$ or $\left.X_{\theta_{s}} X_{\theta}\right)$ is connected, an elementary version of Lang's theorem [20] implies the existence of $z \in Z(U)$ such that $g^{-1} \lambda(g)=z^{-1} \lambda(z)$. Then $g z^{-1}=\lambda\left(g z^{-1}\right)$, so $g z^{-1} \in G_{\lambda}$. By Lemma 1.1, $g \in B$, so $g z^{-1} \in G_{\lambda} \cap B=B_{\lambda}$. Hence $g=g z^{-1} z \in\left\langle B_{\lambda}, Z(U)\right\rangle$, so $N_{G}\left(U_{\lambda}\right) \subseteq\left\langle B_{\lambda}, Z(U)\right\rangle$. The other inclusion is obvious.

LEMma 2.4 Let $z \in Z(U)$ and suppose $\left\langle G_{\lambda}^{s}, z\right\rangle$ is a finite group. Then there exists a positive integer $r$ such that $\left\langle G_{\lambda}^{s}, z\right\rangle \subseteq G_{\lambda r}$.

Proof. First suppose $Z(U)$ is one-dimensional. Thus $Z(U)=$ $\left\langle x_{\theta}(t) \mid t \in k\right\rangle$ where $\theta$ is the root of maximal height in $\Sigma^{+}$. Choose $n \in N \cap\left\langle X_{\theta}, X_{-\theta}\right\rangle$ so that $n x_{\theta}(t) n^{-1}=x_{-\theta}(-t)$. Suppose $z=x_{\theta}(t)$ for some fixed, nonzero, $t \in k$ and put $g=n z$. On the 3 -dimensional adjoint module for $\left\langle X_{\theta}, X_{-\theta}\right\rangle g$ is represented by a matrix whose trace is $t^{2}-1$. Since $g$ has finite order this implies that $t$ is algebraic over $G F(p)$. Suppose $t \in G F\left(p^{r}\right)$ then, since we may suppose that $\lambda\left(x_{\theta}(t)\right)=x_{\theta}\left(t^{q}\right)$, we have $\left\langle G_{\lambda}^{\circ}, z\right\rangle \subseteq G_{\lambda^{r}}$.

Now suppose $Z(U)$ is two-dimensional. First suppose $G$ is of type $C_{l}$ or $F_{4}$. Hence $k$ has characteristic 2 and there exist roots $\left\{\delta_{1}, \delta_{2}, \delta_{1}+\delta_{2}, \delta_{1}+2 \delta_{2}\right\} \subseteq \Sigma^{+}$such that $Z(U)=\left\langle x_{\delta_{1}+\delta_{2}}(t), x_{\delta_{1}+2 \delta_{2}}(t) \mid t \in k\right\rangle$ (in fact $\delta_{1}+\delta_{2}=\theta_{s}$ and $\delta_{1}+2 \delta_{2}=\theta$ ). We suppose $z=x_{\delta_{1+\delta 2}}\left(t_{1}\right) x_{\delta_{1}+2 \delta_{2}}\left(t_{2}\right)$ for some fixed $t_{1}, t_{2} \in k$. Put $G_{1}=\left\langle x_{r}(t) \mid \pm \gamma \in\left\{\delta_{1}, \delta_{2}\right\}, t \in k\right\rangle$ thus $G_{1}$ is of type $C_{2}$ and $\lambda$ fixes $G_{1}$. Choose $n \in\left(G_{1}\right)_{2}$ such that $n x_{\delta_{i}}(t) n^{-1}=$ $x_{-\delta_{i}}(t)$ and put $g=n z$. There is a natural 4-dimensional module for $G_{1}$ on which

$$
n \longrightarrow\left(\begin{array}{llll}
1 & 1 & 1 & 1
\end{array}\right) \text { and } z \longrightarrow\left(\begin{array}{cccc}
1 & 0 & t_{1} & t_{2} \\
& 1 & 0 & t_{1} \\
& & 1 & 0 \\
& & & 1
\end{array}\right)
$$

This gives $t_{1}^{2}$ and $t_{2}$ as coefficients in the characteristic polynomial of $g$. Since $g$ has finite order $t_{1}, t_{2}$ are algebraic over $G F(Z)$ and we are done.

If $G$ is of type $G_{2}, z=x_{2 \alpha_{1}+\alpha_{2}}\left(t_{1}\right) x_{3 \alpha_{1}+2 \alpha_{2}}\left(t_{2}\right)$ and choosing $n \in N_{\lambda}$ such 
that $n x_{\alpha_{i}}(t) n^{-1}=x_{-\alpha_{i}}(-t)$ put $g=n z$. Compute the characteristic polynomial for $g$ as represented in the 7-dimensional module for $G$. Its coefficients are $\left(t_{1}^{2}-1\right)$ and $\left(t_{2}^{2}-t_{1}^{2}+1\right)$. Hence, as before, we are done.

LEMMA 2.5. There exists a positive integer $r$ such that, with $\mu=\lambda^{r}$, we have $G_{\mu}^{s} \subseteq M$ and $U_{\mu} \in \operatorname{Syl}_{p}(M)$.

Proof. Choose the positive integer $r$ to be maximal subject to $G_{\lambda^{r}}^{s} \cong M$. Without loss, we may assume $r=1$, and shall show that $U_{\lambda} \in \operatorname{Syl}_{p}(M)$. Suppose $U_{\lambda} \notin \operatorname{Syl}_{p}(M)$. By Lemma 2.3 and Sylow's theorem, there exists $z \in Z(U)-U_{\lambda}$ such that $\left\langle G_{i}^{s}, z\right\rangle \subseteq M$. By Lemma 2.4, $\left\langle G_{\lambda}^{s}, z\right\rangle \leqq G_{\lambda^{n}}$ for some $n$. Hence the lemma follows from the following statement, which contradicts the maximality of $r$ :

(†) If $z \in Z(U)_{\lambda^{n} \lambda^{n}}-U_{\lambda}$ for some $n$, then $\left\langle G_{\lambda}^{s}, z\right\rangle \supseteqq G_{\lambda^{m}}^{s}$ for some $m>1$.

We now establish (†). Let $K=\left\langle G_{\lambda}^{s}, z\right\rangle$.

Our method is to first study the case $A_{1}$ and use this result along with the action of $N_{\lambda}$ on the root subgroups of $G_{\lambda}$.

Case 0. $\Sigma=A_{1}$ : If $p$ is odd, $(\dagger)$ is an immediate consequence of a result of Dickson [7]. Suppose $p=2$. Then $G_{\lambda}^{(s)}=\left\langle x_{\rho}(t)\right.$, $x_{-\rho}(t)|t \in G F(q)\rangle$ and $z=x_{\rho}\left(t_{1}\right)$ for some $t_{1} \in G F\left(q^{n}\right)-G F(q)$, where $\Sigma^{+}=\{\rho\}$. Define $m$ by $G F(q)\left(t_{1}\right)=G F\left(q^{m}\right)$, so that $K \leqq G_{\lambda} m$ and $m>1$. Now distinct Sylow 2-subgroups in $G_{\lambda^{m}}$ intersect trivially, so distinct Sylow 2-subgroups in $K$ intersect trivially. Since $G_{\lambda} \cong K$ and $G_{\lambda}$ has more than one Sylow 2-subgroup, so does $K$. It follows that any two involutions in $K$ are conjugate in $K$, [13]. In particular, $x_{\rho}\left(t_{1}\right)$ and $x_{\rho}(1)$ are conjugate in $K$, hence conjugate in $N_{K}(U \cap K)$. Hence there are $u \in U, h_{1} \in H$ such that $u h_{1} \in K$ and $x_{\rho}(1)^{u h_{1}}=x_{\rho}\left(t_{1}\right)$. Identifying $H$ with $\operatorname{Hom}\left(\Gamma, k^{*}\right)$, we see that $h_{1}(\rho)=t_{1}^{1 / 2}$. Hence for any positive integer $l$, and any $t \in G F(q)$, we may choose $h \in K$ such that $x_{\rho}(1)^{h}=x_{\rho}(t)$, and conclude that $x_{\rho}\left(t t_{1}^{l}\right)=x_{\rho}(1)^{h\left(u h_{1}\right)^{l}} \in K$. Thus $x_{\rho}\left(f\left(t_{1}\right)\right) \in K$ for all $f[X] \in G F(q)[X]$. Hence $x_{\rho}(t) \in K$ for all $t \in G F\left(q^{m}\right)$, i.e., $U_{\lambda^{m}} \cong K$. Then $K \supseteqq\left\langle U_{\lambda^{m}}, N_{\lambda}\right\rangle \supseteqq G_{\lambda^{m}}^{s}$ as required.

Case 1. $\Sigma$ arbitrary, $\lambda=\sigma_{q}$, and $Z(U)=X_{\theta}$ : Let $G_{\theta}=\left\langle X_{\theta}, X_{-\theta}\right\rangle$ and $K_{\theta}=K \cap G_{\theta}$. Then $\lambda$ is an endomorphism of $G_{\theta}$, and $\left\langle\left(G_{\theta}\right)_{\lambda}, z\right\rangle \leqq$ $K_{\theta} \leqq\left(G_{\theta}\right)_{\lambda^{n}}$ since $z \in Z(U)=X_{\theta}$. By Case $0,\left(G_{\theta}\right)_{\lambda^{m}} \leqq K_{\theta}$ for some $m>1$, so $\left(X_{\theta}\right)_{\lambda^{m}} \subseteq K$. Conjugating by elements of $N_{\lambda}$, we get $\left(X_{\rho}\right)_{\lambda^{m}} \subseteq K$ for all $\rho \in \Sigma$ of the same length as $\theta$. If there is one root length, this gives immediately $G_{i}^{s} \leqq K$. If there are two root 
lengths, let $\rho \in \Sigma$ be short and choose $\sigma \in \Sigma$ long such that $\rho+\sigma \in \Sigma$. For any $t \in G F\left(q^{m}\right), t \neq 0, h_{\sigma}(t) \in K$, so $x_{\rho}\left(t^{-1}\right)=x_{\rho}(1)^{h_{\sigma}(t)} \in K$. Thus $\left(X_{\rho}\right)_{\lambda^{m}} \subseteq K$, so $K \supseteqq\left\langle\left(X_{\rho}\right)_{\lambda^{m}} \mid \rho \in \Sigma\right\rangle=G_{\lambda^{m}}^{s}$.

Case 2. $\lambda=\sigma_{q}, Z(U) \neq X_{\theta}$ : We have two root length, $Z(U)=$ $\left\langle X_{\theta_{g}}, X_{\theta}\right\rangle$, and the characteristic of $k$ is the strength of the multiple bond in the Dynkin diagram of $\Sigma$. Let $\Sigma^{0}=\left(Z \theta_{s}+Z \theta\right) \cap \Sigma, G^{0}=$ $\left\langle X_{\rho} \mid \rho \in \Sigma^{0}\right\rangle, K^{0}=G^{0} \cap K$. Then $\lambda$ is an endomorphism of $G^{0},\left\langle\left(G^{0}\right)_{\lambda}^{s}, z\right\rangle \subseteq$ $K^{0}$. If $(\dagger)$ holds for $G^{0}$, then $\left\langle\left(G^{0}\right)_{\lambda}^{s}, z\right\rangle \supseteq\left(G^{0}\right)_{\lambda}^{s}$ for some $m>1$. In particular, $\left(X_{\rho}\right)_{\lambda^{m}} \leqq K$ for $\rho=\theta_{s}$ and $\theta$, and then for all $\rho \in \Sigma$, by conjugation by elements of $N_{\lambda}$. Hence in proving ( $\dagger$ ) we may assume $\Sigma=\Sigma^{0}$. Thus $\Sigma=C_{2}$ or $G_{2}$, with $p=2$ or 3 respectively.

We take $\Pi=\{\alpha, \beta\}$, with $\alpha$ long and $\beta$ short. Suppose $\Sigma=C_{2}$, so $p=2$. For every $y=x_{\alpha+\beta}\left(t_{1}\right) x_{\alpha+2 \beta}\left(t_{2}\right) \in Z(U)$, set $\pi_{\alpha+\beta}(y)=t_{1}$, $\pi_{\alpha+2 \beta}(y)=t_{2}$. Let $k_{1}=\pi_{\alpha+\beta}(K \cap Z(U)), k_{2}=\pi_{\alpha+2 \beta}(K \cap Z(U))$. Thus $k_{i}$ is an additive group, $G F(q) \subseteq k_{i} \subseteq G F\left(q^{n}\right), i=1$, 2, and $k_{1} \cup k_{2} \neq G F(q)$ as $z \notin U_{\lambda}$. Let $t_{1} \in k_{1}, t_{2} \in k_{2}$, and choose $u_{1}=x_{\alpha+\beta}\left(t_{1}\right) x_{\alpha+2 \beta}\left(t_{1}^{\prime}\right) \in K$ and $u_{2}=x_{\alpha+\beta}\left(t_{2}^{\prime}\right) x_{\alpha+2 \beta}\left(t_{2}\right) \in K$. Now $n_{\alpha}(1), n_{\beta}(1) \in G_{\lambda}^{s} \subseteq K$, so

$$
x_{\alpha+\beta}\left(t_{1} t_{2}\right) x_{\alpha+2 \beta}\left(t_{1}^{2} t_{2}\right)=\left[u_{1}^{n} \alpha^{(1)}, u^{n_{\beta}(1)}\right] \in K .
$$

Thus $t_{1} t_{2} \in k_{1}, t_{1}^{2} t_{2} \in k_{2}$, so $\left\{t^{2} \mid t \in k_{1}\right\} \subseteq k_{2} \subseteq k_{1}$, from the special cases $t_{2}=1$ and $t_{1}=1$. But the map $t \rightarrow t^{2}$ is injective on $G F\left(q^{n}\right)$, so $k_{1}=$ $k_{2}$. From (1), $k_{1} \cdot k_{2} \subseteq k_{1}$, so $k_{1}$ is a field. Thus for some $m>1, k_{1}=$ $k_{2}=G F\left(q^{m}\right)$. For any $t \in G F\left(q^{m}\right)$, we take $t_{1}=t$ and $t_{2}=t^{-1}$ and $t^{-2}$ in (1) and conclude $\left\langle\left(X_{\alpha+\beta}\right)_{\lambda m},\left(X_{a+2 \beta}\right)_{\lambda m}\right\rangle \subseteq K$. As usual this gives $G_{\lambda m}^{s} \cong K$.

Suppose $\Sigma=G_{2}$ so $p=3$. Write $z=u_{1} u_{2}$, with $u_{1} \in X_{\alpha+2 \beta}$ and $u_{2} \in X_{2 \alpha+3 \beta}$. Then $u_{2}=\left[z^{n} \alpha^{(1)}, x_{\alpha}(1)\right]^{ \pm 1} \in K$, so $u_{1}=z u_{2}^{-1} \in K$. Since $z \notin$ $G_{\lambda}$, either $u_{1}$ or $u_{2} \notin G_{\lambda}$, so without loss we may assume $z=u_{1}$ or $z=u_{2}$.

Since $G$ has a graph automorphism commuting with $\lambda$ and interchanging $\theta_{s}$ and $\theta$ we may assume that $z \in X_{2 \alpha+3 \beta}$. By Case 0 applied to $\left\langle X_{2 \alpha+3 \beta}, X_{-2 \alpha-3 \beta}\right\rangle$, there is $m>1$ such that $\left(X_{\rho}\right)_{\lambda^{m}} \subseteq K$ for $\rho=$ $2 \alpha+3 \beta$, and then for all long $\rho \in \Sigma$. For any $t \in G F\left(q^{m}\right), K$ contains $\left[x_{\alpha}(t), x_{\beta}(1), x_{\beta}(1)\right]=x_{\alpha+2 \beta}( \pm t) x_{\alpha+3 \beta}\left(t^{\prime}\right) x_{2 \alpha+3 \beta}\left(t^{\prime \prime}\right)$ with $t^{\prime}, t^{\prime \prime} \in G F\left(q^{m}\right)$, so $x_{\alpha+2 \beta}(t) \in K$ as $\alpha+3 \beta$ and $2 \alpha+3 \beta$ are long. Thus $\left(X_{\rho}\right)_{\lambda^{m}} \subseteq K$ for $\rho=\alpha+2 \beta$, hence for all short $\rho$, whence $G_{\lambda m}^{s} \leqq K$.

Case 3. $\lambda={ }^{2} \sigma_{q}$ or ${ }^{3} \sigma_{q}$, with $G_{\lambda}$ a Steinberg variation, but $\Sigma \neq$ $A_{2 n}$ (the cases of twisted $F_{4}, G_{2}, C_{2}$ are not being considered here): In this case $Z(U)=X_{\theta}$, so by Case $0, K \supseteqq\left(X_{\theta}\right)_{\lambda m}$ for some $m>1$. Conjugating by $N_{\lambda}$, we get $K \supseteqq\left(X_{\rho}\right)_{\lambda^{m}}$ for all $\rho \in \Sigma$ fixed by the twist defining $G$. Choose such a $\rho$ and a $\sigma$ not fixed by the twist, 
such that $(\rho, \sigma)<0$ (these can be found in $\Pi$, for example, joined by the multiple bond in the twisted Dynkin diagram). Denote the images of $\sigma$ under the twist by $\sigma_{1}$ (and also $\sigma_{2}$ if $G_{\lambda}={ }^{3} D_{4}$ ). Then $x_{o}(t) x_{\sigma_{1}}\left(t^{q}\right)\left(\cdot x_{\sigma_{2}}\left(t^{q^{2}}\right)\right) \in K$ for all $t \in G F\left(q^{2}\right)\left(G F\left(q^{3}\right)\right)$. Since $K \supseteqq\left\langle\left(X_{\rho}\right)_{\lambda^{m}}\right.$, $\left.\left(X_{-\rho}\right)_{\lambda^{m}}\right\rangle, h_{\rho}(t) \in K$ for all $t \in G F\left(q^{m}\right), t \neq 0$.

If $G_{2}={ }^{3} D_{4}$ and $m \equiv 1(\bmod 3)$, then for all $t \in G F\left(q^{3}\right)$ and all $0 \neq$ $u \in G F\left(q^{m}\right)$, we have $\left(x_{\sigma}(t) x_{\sigma_{1}}\left(t^{q}\right) x_{\sigma_{2}}\left(t^{q^{2}}\right)\right)^{h} \rho^{\left(u^{-1}\right)}=x_{\sigma}(t u) x_{\sigma_{1}}\left(t^{q} u\right) x_{\sigma_{2}}\left(t^{q^{2}} u\right)=$ $x_{o}(t u) x_{\sigma_{1}}\left((t u)^{q^{m}}\right) x_{\sigma_{2}}\left((t u)^{q^{2 m}}\right) \in K$. Hence $x_{o}(v) x_{\sigma_{1}}\left(v^{q^{m}}\right) x_{\sigma_{2}}\left(v^{q^{2 m}}\right) \in K$ for all $v$ of the form $\sum_{i} t_{i} u_{i}$ with $t_{i} \in G F\left(q^{3}\right), u_{i} \in G F\left(q^{m}\right)$, that is, for all $v \in$ $G F\left(q^{3 m}\right)$. Thus $\left(X_{\sigma} X_{\sigma_{1}} X_{\sigma_{2}}\right)_{\lambda^{m}} \subseteq K$, so $G_{\lambda m}^{s} \subseteq K$. The case $m \equiv-1$ $(\bmod 3)$ is similar, as is the case $\lambda={ }^{2} \sigma_{q}$ and $m$ odd.

If $G_{\lambda}={ }^{3} D_{4}$ and $m \equiv 0(\bmod 3)$, we may assume $m=3$, and must prove $x_{\sigma}(t) \in K$ for all $t \in G F\left(q^{3}\right)$. Now

$$
\begin{aligned}
x(t, u) & \equiv x_{\sigma_{1}}\left(\left(u^{q}-u\right) t^{q}\right) x_{\sigma_{2}}\left(\left(u^{q^{2}}-u\right) t^{q^{2}}\right) \\
& =\left(x_{\sigma}(t u) x_{\sigma_{1}}\left((t u)^{q}\right) x_{\sigma_{2}}\left((t u)^{q^{2}}\right)\right)^{-1}\left(x_{\sigma}(t) x_{\sigma_{1}}\left(t^{q}\right) x_{\sigma_{2}}\left(t^{q^{2}}\right)\right)^{h^{\rho^{(u-1}}} \in K
\end{aligned}
$$

for all $t, u \in G F\left(q^{3}\right)$, so for all $t, u, v \in G F\left(q^{3}\right)$ with $u, v \notin G F(q), K$ contains $x(t, u)^{h} \rho^{\left(\left(v^{q}-v\right)^{-1}\left(u^{q}-u\right)\right)} \cdot x(t, v)^{-1}=x_{o_{2}}\left(y(u, v) t^{q^{2}}\right)$, where $y(u, v)=$ $\left(u^{q^{2}}-u\right)\left(v^{q}-v\right)\left(u^{q}-u\right)^{-1}-\left(v^{q^{2}}-v\right)$.

Clearly there exist $u, v \in G F\left(q^{3}\right)-G F(q)$ such that $y(u, v) \neq 0$; fixing these and letting $t$ vary, we get $x_{\sigma_{2}}(t) \in K$ for all $t \in G F\left(q^{3}\right)$, as desired. The case $\lambda={ }^{2} \sigma_{q}, m$ even, is similar but simpler: $x_{\sigma_{1}}\left(\left(u^{q}-u\right) t^{q}\right) \in K$ for $t, u \in G F\left(q^{2}\right)$, and $u$ may be chosen so $u^{q}-u \neq 0$.

Case 4. $\Sigma=A_{n}^{2}, \lambda={ }^{2} \sigma_{q}$ : For each $\rho \in \Sigma$, let $\rho_{1}$ be the image of $\rho$ under the twist. If $\rho \in \Sigma$ and $\rho+\rho_{1} \in \Sigma$, then $G_{\lambda}$ has a nonabelian "root subgroup" $\left\{x_{\rho}(t) x_{\rho_{1}}\left(t^{q}\right) x_{\rho+\rho_{1}}(u) \mid t, u \in G F\left(q^{2}\right), t^{1+q}+u+u^{q}=0\right\}$. If $\rho \in \Sigma$ and $\rho+\rho_{1} \notin \Sigma$, then $G_{\lambda}$ has an abelian root subgroup

$$
\left\{x_{\rho}(t) x_{\rho_{1}}\left(t^{q}\right) \mid t \in G F\left(q^{2}\right)\right\} \text {. }
$$

There exists $\tau \in \Sigma^{+}$such that $\tau+\tau_{1}=\theta$. Thus $\left(X_{\theta}\right)_{\lambda}=\left\{x_{\theta}(u) \mid u \in\right.$ $\left.G F\left(q^{2}\right), u+u^{q}=0\right\}$. Choose $0 \neq u_{0} \in G F\left(q^{2}\right)$ such that $u_{0}+u_{0}^{q}=0$. Then for any $u \in G F\left(q^{2}\right), u+u^{q}=0$ if and only if $u u_{0}^{-1} \in G F(q)$, so $\left(X_{\theta}\right)_{2}=\left\{x_{\theta}\left(u_{0} u_{1}\right) \mid u_{1} \in G F(q)\right\}$. Let $K_{\theta}=K \cap\left\langle X, X_{-\theta}\right\rangle_{\lambda}$, so that $K_{\theta}$ contains $\left(X_{\theta}\right)_{\lambda},\left(X_{-\theta}\right)_{\lambda}$, and $z$. Let $h=h_{\theta}\left(u_{0}\right) \in H$. Then $K_{\theta}^{h}$ contains $\left\{x_{ \pm \theta}\left(u_{1}\right) \mid u_{1} \in G F(q)\right\}$, canonical generators of $A_{1}(q)$, and also contains $z^{h}=x_{\theta}(t)$ for some $t \notin G F(q)$. By Case 0 , there exists $m>1$ such that $K_{\theta}^{h}$ contains $\left\{x_{ \pm \theta}\left(u_{1}\right) \mid u_{1} \in G F\left(q^{m}\right)\right\}$. In particular, $K_{\theta}$ contains $x_{ \pm \theta}\left(u_{1}\right)^{h^{-1}}=x_{ \pm \theta}\left(u_{0} u_{1}\right)$ for all $u_{1} \in G F\left(q^{m}\right) \cdot h_{\theta}\left(u_{1}\right) \in K_{\theta}^{h}$ for all $u_{1} \in G F\left(q^{m}\right)$, so $h_{\theta}\left(u_{1}\right)=h_{\theta}\left(u_{1}\right)^{h^{-1}} \in K_{\theta}$ for all $u_{1} \in G F\left(q^{m}\right), u_{1} \neq 0$. For any $t, u \in$ $G F\left(q^{2}\right)$ satisfying $t^{1+q}+u+u^{q}=0$ and any $u_{1} \in G F\left(q^{m}\right)^{x}$, we conjugate $x_{\tau}(t) x_{\tau_{1}}\left(t^{q}\right) x_{\theta}(u)\left(\in G_{\lambda}\right)$ by $h_{\theta}\left(u_{1}\right)$ and get

$$
x\left(t, u, u_{1}\right)=x_{\tau}\left(t u_{1}\right) x_{\tau_{1}}\left(t^{q} u_{1}\right) x_{\theta}\left(u u_{1}^{2}\right) \in K .
$$


Suppose $m$ is odd. Then $t^{q} u_{1}=\left(t u_{1}\right)^{q^{m}}$ and $t u_{1}\left(t u_{1}\right)^{q^{m}}+u u_{1}^{2}+$ $\left(u u_{1}^{2}\right)^{q^{m}}=t u_{1} t^{q} u_{1}+u u_{1}^{2}+u^{q} u_{1}^{2}=\left(t^{1+q}+u+u^{q}\right) u_{1}^{2}=0$, so $x\left(t, u, u_{1}\right) \epsilon$ $G_{\lambda^{m}}$. Now every element of $G F\left(q^{2 m}\right)$ is a sum of elements of the form $t u_{1}$ with $t \in G F\left(q^{2}\right), u_{1} \in G F\left(q^{m}\right)^{x}$, so for every $t \in G F\left(q^{2 m}\right), K$ contains an element of the form $x_{\tau}(t) x_{\tau_{1}}\left(t^{q^{m}}\right) x_{\theta}(u)$ with $t^{1+q^{m}}+u+u^{q^{m}}=$ 0 . Since $K$ contains $x_{\theta}\left(u_{0} u_{1}\right)$ for all $u_{1} \in G F\left(q^{m}\right)$, it contains $x_{\theta}(v)$ for all $v \in G F\left(q^{2 m}\right)$ satisfying $v+v^{q^{m}}=0$. Hence $K$ contains $\left\{x_{\rho}(t) x_{\rho_{1}}\left(t^{q^{m}}\right) x_{\theta}(u) \mid t\right.$, $\left.u \in G F\left(q^{2 m}\right), t^{1+q^{m}}+u+u^{q^{m}}=0\right\}$, a nonabelian root subgroup of $G_{\lambda^{m}}$. Conjugating by $N_{\lambda}$, we see that $K$ contains all nonabelian root subgroups of $G_{\lambda^{m}}$. If $n=1$, we are therefore done. If $n>1$, there exists $\gamma \in \Sigma$ such that $\gamma+\gamma_{1} \notin \Sigma$ while $\gamma+\theta, \gamma_{1}+\theta \in \Sigma$ (for example, $-\gamma \in \Pi$, with $-\gamma$ at an end of the Dynkin diagram). Then for all $t \in G F\left(q^{2}\right), u_{1} \in G F\left(q^{m}\right)^{x}$, we have $x_{\gamma}\left(t u_{1}\right) x_{\gamma_{1}}\left(\left(t u_{1}\right)^{q^{m}}\right)=x_{\gamma}\left(t u_{1}\right) x_{\gamma_{1}}\left(t^{q} u_{1}\right)=$ $\left(x_{\gamma}(t) x_{\gamma_{1}}\left(t^{q}\right)\right)^{h^{\left(u_{1}\right)}} \in K$. It follows that $x_{\gamma}(v) x_{\gamma_{1}}\left(v^{q}\right)^{m} \in K$ for all $v \in G F\left(q^{2 m}\right)$, so $K$ contains an abelian root subgroup of $G_{\lambda^{m}}$. Hence $K \supseteqq G_{\lambda m}^{s}$, as required.

Suppose $m$ is even. We may assume $m=2$, and shall prove $G_{\lambda^{2}}^{s} \supseteq K$. Let $\tau, \gamma$ be as in the previous paragraph. For any $t \in$ $G F\left(q^{2}\right)$ and $u_{1} \in G F\left(q^{2}\right)^{x}$, we have $x_{1}=x_{\gamma}\left(t u_{1}\right) x_{\gamma_{1}}\left(t^{q} u_{1}\right)=\left(x_{\gamma}(t) x_{\gamma_{1}}\left(t^{q}\right)\right)^{h_{\theta}\left(u_{1}\right)} \in$ $K$, and also $\left.x_{2}=x_{\gamma}\left(t u_{1}\right) x_{r_{1}}\left(t u_{1}\right)^{q}\right) \in G_{\lambda} \subseteq K$. Hence $x_{\gamma_{1}}\left(t^{q}\left(u_{1}^{q}-u_{1}\right)\right)=$ $x_{2} x_{1}^{-1} \in K$. Fix $u_{1}$ such that $u_{1}^{q} \neq u_{1}$ and let $t$ vary; we get $\left(X_{\gamma_{1}}\right)_{\lambda^{2}} \subseteq$ $K$. Similarly, $\left(X_{\gamma}\right)_{\lambda^{2}} \subseteq K$, so conjugating by $N_{\lambda}$, we get $\left(X_{\rho}\right)_{\lambda^{2}} \cong K$ for all $\rho \in \Sigma$ such that $\rho+p_{1} \notin \Sigma$. Also, we have $x_{\theta}\left(u_{0} u_{1}\right) \in K$ for all $u_{1} \in G F\left(q^{2}\right)$. Since $u_{0}$ was chosen in $G F\left(q^{2}\right)$ and $u_{0} \neq 0,\left(X_{\theta}\right)_{\lambda^{2}} \subseteq K$. Hence $\left(X_{\theta}\right)_{\lambda^{2}} \subseteq K$ for all $\rho \in \Sigma$ with $\rho=\rho_{1}$. For any $t \in G F\left(q^{2}\right)$ there is $u \in G F\left(q^{2}\right)$ such that $x_{3}=x_{\tau}(t) x_{\tau_{1}}\left(t^{q}\right) x_{\theta}(u) \in G_{\lambda^{\prime}}$. Let $u_{1} \in G F\left(q^{2}\right)^{x}$. Let $x_{4}=x_{3}^{h \theta_{\left(u_{1}\right)}}=x_{\tau}\left(t u_{1}\right) x_{\tau_{1}}\left(t^{q} u_{1}\right) x_{\theta}() \in K$ and choose $u^{\prime} \in G F\left(q^{2}\right)$ such that $x_{5}=x_{\tau}\left(t u_{1}\right) x_{\tau_{1}}\left(\left(t u_{1}\right)^{q}\right) x_{\theta}\left(u^{\prime}\right) \in G_{\lambda^{\prime}}$. Then $x_{\tau_{1}}\left(t^{q}\left(u_{1}^{q}-u_{1}\right)\right)=x_{5} x_{4}^{-1} x_{\theta}() \in$ $K$. As above, we get $\left(X_{\tau_{1}}\right)_{\lambda^{2}} \subseteq K$. Conjugating by $N_{\lambda^{\prime}},\left(X_{\rho}\right)_{\lambda^{2}} \subseteq K$ for all $\rho \in \Sigma$ such that $\rho+\rho_{1} \in \Sigma$. Thus $\left(X_{\rho}\right)_{\lambda^{2}} \subseteq K$ for all $\rho \in \Sigma$, as required.

Case 5. $\Sigma=C_{2}, \lambda={ }^{2} \sigma_{q}, q>2$ : Thus $q=2 q_{0}^{2}, q_{0}=2^{j}>1$. We take $\Pi=\{\alpha, \beta\}$, with $\beta$ short. Let $\mathscr{S}$ be the additive group $k \oplus k$. For $\left(t_{1}, t_{2}\right) \in \mathscr{S}$, set $x\left(t_{1}, t_{2}\right)=x_{\alpha+\beta}\left(t_{1}\right) x_{\alpha+2 \beta}\left(t_{2}\right)$. For any subgroup $J$ of $G$ set $\mathscr{S}_{J}=\left\{\left(t_{1}, t_{2}\right) \mid x\left(t_{1}, t_{2}\right) \in J\right\}$, an additive subgroup of $\mathscr{S}_{\text {. Thus }}$ $\mathscr{S}_{G_{\lambda}}=\left\{\left(t, t^{2 q_{0}}\right) \mid t \in G F(q)\right\}$. Since $z \in Z\left(U_{\lambda}\right)-G_{\lambda}, \mathscr{S}_{G_{\lambda}} \subset \mathscr{S}_{K} \cong \mathscr{S}_{G_{\lambda n}}$. Also, let $n_{0}=\left(n_{\alpha}(1) n_{\beta}(1)\right)^{2} \in G_{\lambda}$, so that $x_{\rho}(t)^{n_{0}}=x_{-\rho}(t)$ for all $\rho \in \Sigma$, $t \in k$, and also $n_{0}^{2}=1$. Finally, for any $t_{1}, t_{2} \in k^{x}$, let $h\left(t_{1}, t_{2}\right)$ be the element of $H$ which takes $\alpha$ to $t_{1}^{2} t_{2}^{-1}$ and $\beta$ to $t_{1}^{-1} t_{2}$. Thus $x\left(t_{1}, t_{2}\right)^{h\left(u_{1}, u_{2}\right)}=$ $x\left(t_{1} u_{1}, t_{2} u_{2}\right)$.

Suppose $\left(t_{1}, t_{2}\right) \in \mathscr{S}_{K}$ and $t_{1} t_{2} \neq 0$. We show that $h\left(t_{1}, t_{2}\right) \in K$. First $C_{G}\left(x\left(t_{1}, t_{2}\right)\right) \subseteq B$, for if $g \in C_{G}\left(x\left(t_{1}, t_{2}\right)\right)$, we write $g=b n u$ in canonical form and get $x\left(t_{1}, t_{2}\right)^{n} \in X_{\alpha+\beta} X_{\alpha+2 \beta}$, so $n \in H$ and $g \in B$. On the other 
hand, $C_{U}\left(n_{0}\right)=1$ as $U \cap U^{n_{0}}=1$. Hence $x\left(t_{1}, t_{2}\right)$ and $n_{0}$ do not centralize any involution of $G$ in common. If follows that $x\left(t_{1}, t_{2}\right)$ and $n_{0}$ are conjugate in the (dihedral) group $\left\langle x\left(t_{1}, t_{2}\right), n_{0}\right\rangle$, hence also in $K$. Similarly, $x(1,1)$ and $n_{0}$ are conjugate in $K$. Thus $x\left(t_{1}, t_{2}\right)=x(1,1)^{g}$ for some $g \in K$. Writing $g$ in canonical form, we see $g=u h\left(t_{1}, t_{2}\right)$ for some $u \in U$. However, $B \cap K=(U \cap K)(H \cap K)$. To see this, choose $t \in G F(q), t \neq 0$ or 1 , and let $h=h\left(t, t^{2 q_{0}}\right) \in G_{\lambda} \subseteq K$. Then $C_{U}(h)=1$, so $C_{B}(h)=H$. By the Schur-Zassenhaus theorem, $B \cap K$ has a subgroup $H_{0}$ such that $B \cap K=(U \cap K) H_{0}, U \cap K \cap H_{0}=1$, and $h \in H_{0}$. Then $H_{0}$ is abelian, so $H_{0} \subseteq C_{B}(h)=H$, so $H_{0}=H \cap K$. Since $g \in B \cap K, h\left(t_{1}, t_{2}\right) \in H \cap K \subseteq K$, as claimed.

Thus, if $\left(t_{1}, t_{2}\right) \in \mathscr{S}_{K},\left(u_{1}, u_{2}\right) \in \mathscr{S}_{K}$, and $u_{1} u_{2} \neq 0$, then $\left(t_{1}, u_{1}, t_{2} u_{2}\right) \in$ $\mathscr{S}_{K}$.

Suppose now that no element of $\mathscr{S}_{K}$ has the form $(0, t)$ or $(t, 0)$ with $t \neq 0$. Let $\mathscr{S}_{1}=\left\{t \mid(t, u) \in \mathscr{S}_{K}\right.$ for some $\left.u\right\}$, and define the function $\phi$ on $\mathscr{S}_{1}$ by the condition $(t, \varphi(t)) \in \mathscr{S}_{K}$. Since $\mathscr{S}_{1}$ is an additive subgroup of $G F\left(q^{n}\right)$, and $G F(q) \subset \mathscr{S}_{1}$, the last paragraph implies that $\mathscr{S}_{1}$ is a field, so $\mathscr{S}_{1}=G F\left(q^{m}\right)$ for some $m>1$; also, $\varnothing$ preserves multiplication, so is an automorphism of $G F\left(q^{m}\right)$. Thus for some $d=2^{i}, d \leqq q^{m}, \mathscr{S}_{K}=\left\{\left(t, t^{d}\right) \mid t \in G F\left(q^{m}\right)\right\}$. Since $\mathscr{S}_{G_{\lambda}} \subseteq \mathscr{S}_{K}, t^{d}=t^{2 q_{0}}$ for all $t \in G F(q)$. Let $x_{0}=x_{\alpha}(1) x_{\beta}(1) x_{\alpha+\beta}(1)\left(\in G_{\lambda}\right)$. For each $t, u \in$ $G F\left(q^{m}\right)^{x}, K$ contains $\left[x_{0}^{h\left(t, t^{d}\right)}, x_{0}^{h\left(u, u^{d}\right)}\right]=x\left(w_{1}, w_{2}\right)$ where $w_{1}=t^{2-d} u^{d-1}+$ $u^{2-d} t^{d-1}, w_{2}=t^{2-d} u^{2 d-2}+u^{2-d} t^{2 d-2}$. By the above $w_{2}=w_{1}^{d}$. In the special case $u=1$ this yields $\left(t^{-d}+t^{-d^{2}+2 d-2}\right)\left(t^{d^{2}}+t^{2}\right)=0$. Fix $t$. We wish to show $t^{d^{2}}+t^{2}=0$. Suppose $t^{d^{2}}+t^{3 d-2}=0$. For any $u \in G F(q), u^{d}=$ $u^{2 q_{0}}$; with the equation $w_{2}=w_{1}^{d}$, this gives $\left(t^{2-d}+t^{2 d-2}\right)\left(u^{1-q_{0}}+u^{2 q_{0}-1}\right)^{2}=$ 0 for all $u \in G F(q)^{x}$. Since $q>2$, also $q-1>3 q_{0}-2$, so for suitable $u$, the right hand factor does not vanish. Thus $t^{2-d}=t^{2 d-2}$. Hence $t^{2}+t^{d^{2}}=t^{2}+t^{3 d-2}=0$ anyway. So $t^{2}=t^{d^{2}}$ for all $t \in G F\left(q^{m}\right)$. Let $d_{0}=1 / 2 d$; then $t^{2 d_{0}^{2}}=t$, which implies that $m$ is odd and $H \cap K \supseteqq$ $\left\{h\left(t, t^{2 d_{0}}\right) \mid t \in G F\left(q^{m}\right)\right\}=H_{\lambda^{m}}$. Conjugating elements of $U_{\lambda}$ by those of $H_{\lambda^{m}}$, we find $U_{\lambda^{m}} \leqq K$, so $K \supseteqq\left\langle U_{\lambda^{m}}, n_{0}\right\rangle=G_{\lambda^{m}}^{s}$.

Finally, suppose $\mathscr{S}_{K}$ contains an element of the form $(t, 0)$ or $(0, t)$ for some $t \neq 0$. We show that $K \supseteqq G_{\lambda^{2}}$. This is equivalent to $K^{2} \supseteqq G_{\lambda^{2}}$, so without loss we may assume $(0, t) \in \mathscr{S}_{K}$, i.e., $x_{\alpha+2 \beta}(t) \epsilon$ $K$. Then $K \supseteqq\left\langle x_{\alpha+2 \beta}(t), n_{0}\right\rangle$ so $g=n_{0}(1) x_{\alpha+2 \beta}(t)=n_{\alpha}(1) n_{\alpha+2 \beta}(1) x_{\alpha+2 \beta}(t) \epsilon$ $K$. A $2 \times 2$ matrix calculation shows that $n_{\alpha+2 \beta}(1) x_{\alpha+2 \beta}(t)$ has odd order $e$. Since it commutes with $n_{\alpha}(1), n_{\alpha}(1)=n_{\alpha}(1)^{e}=g^{e} \in K$. For any $u, v \in G F(q), x\left(u, u^{2 q_{0}}\right) \in K$ and $x_{0}(v)=x_{\alpha}(v) x_{\beta}\left(v^{q_{0}}\right) x_{\alpha+\beta}\left(v^{1+q_{0}}\right) \in K$, so $x\left(u v, u^{2} v\right)=\left[x\left(u, u^{2 q_{0}}\right)^{n} \alpha^{(1)}, x_{0}(v)\right] \in K$. Replacing $u$ by $u v$ and $v$ by 1 , we get $x\left(u v, u^{2} v^{2}\right) \in K$, so $x_{\alpha+2 \beta}\left(u^{2}\left(v^{2}+v\right)\right) \in K$. Since $q>2, v$ exists with $v^{2}+v \neq 0$; this gives $\left(X_{\alpha+2 \beta}\right)_{\lambda^{2}} \subseteq K$. It follows easily that $\left(X_{\alpha+\beta}\right)_{\lambda^{2}} \subseteq K$. Hence $n_{\alpha+\beta}(1) \in\left\langle\left(X_{\alpha+\beta}\right)_{\lambda^{2}}, n_{0}\right\rangle \subseteq K$, so $K \supseteqq\left\langle\left(X_{\alpha+\beta}\right)_{\lambda^{2}}\right.$, $\left.n_{\alpha}(1), n_{\alpha+\beta}(1), n_{0}\right\rangle=G_{\lambda^{2}}$. 
Case 6. $\Sigma=F_{4}, \lambda={ }^{2} \sigma_{q}$ : Here $q=2 q_{0}^{2}, q_{0}=2^{j}$. We notate elements of $\Sigma$ as in Lemma 2.1. Then $\Sigma^{+}$is partitioned into 4 subsets giving root subgroups of $U_{\lambda}$ of type ${ }^{2} C_{2}(\{0100,0010,0110,0210\},\{0011$, $1100,1111,2211\},\{0211,1110,1321,2431\}$, and $\{0111,2210,2321,2432\})$ and 4 subsets giving root subgroups of type $A_{1}(\{1000,0001\},\{1210$, $0221\},\{1211,2221\}$, and $\{1221,2421\}) . \quad Z(U)=X_{2321} X_{2432}$. Let $\mathscr{S}=$ $k \oplus k$, for each $\left(t_{1}, t_{2}\right) \in \mathscr{S}$ set $x\left(t_{1}, t_{2}\right)=x_{2321}\left(t_{1}\right) x_{2432}\left(t_{2}\right)$, and for each subgroup $J$ of $G$ set $\mathscr{S}_{J}=\left\{\left(t_{1}, t_{2}\right) \in \mathscr{S} \mid x\left(t_{1}, t_{2}\right) \in J\right\}$. Thus $\mathscr{S}_{G_{2}}=$ $\left\{\left(t, t^{2 q_{0}}\right) \mid t \in G F(q)\right\}$, where $q=2 q_{0}^{2}$, and $\mathscr{S}_{G_{\lambda}} \subset \mathscr{S}_{K} \subseteq \mathscr{S}_{G_{\lambda n}}$.

We show that if $\left(t_{1}, t_{2}\right),\left(u_{1}, u_{2}\right) \in \mathscr{S}_{K}$, then $\left(t_{2} u_{1}, t_{1}^{2} u_{2}\right) \in \mathscr{S}_{K}$. Namely, conjugating $x\left(t_{1}, t_{2}\right)$ and $x\left(u_{1}, u_{2}\right)$ by appropriate elements of $N_{\lambda}(\subseteq K)$, we get $x_{0110}\left(t_{1}\right) x_{0210}\left(t_{2}\right), x_{1111}\left(u_{1}\right) x_{2211}\left(u_{2}\right) \in K$, so $x\left(t_{2} u_{1}, t_{1}^{2} u_{2}\right)=\left[x_{0110}\left(t_{1}\right) x_{0210}\left(t_{2}\right)\right.$, $\left.x_{1111}\left(u_{1}\right) x_{2211}\left(u_{2}\right), x_{1000}(1) x_{0001}(1)\right] \in K$. In particular, since $(1,1) \in \mathscr{S}_{K}$, the $\operatorname{map} \varphi:\left(t_{1}, t_{2}\right) \rightarrow\left(t_{2}, t_{1}^{2}\right)$ is a permutation of $\mathscr{S}_{K}$. For $\left(t_{1}, t_{2}\right),\left(u_{1}, u_{2}\right) \in \mathscr{S}_{K}$, let $\left(z_{1}, z_{2}\right)=\phi^{-1}\left(t_{1}, t_{2}\right)$. Then $\left(t_{1} u_{1}, t_{2} u_{2}\right)=\left(z_{2} u_{1}, z_{1}^{2} u_{2}\right) \in \mathscr{S}_{K}$, so $\mathscr{S}_{K}$ is closed under multiplication. Since $\varphi$ maps $\mathscr{S}_{K}$ to itself, $\mathscr{S}_{K} \subseteq G F\left(q^{m}\right) \oplus G F\left(q^{m}\right)$ for some $m$, and $\mathscr{S}_{K}$ projects onto both summands.

If $\mathscr{S}_{K}$ contains no element of the form $(0, t)$ or $(t, 0)$ for $t \neq 0$, then the map $\psi: G F\left(q^{m}\right) \rightarrow G F\left(q^{m}\right)$ defined by $(t, \psi(t)) \in \mathscr{S}_{K}$ is an automorphism of $G F\left(q^{m}\right)$, so $\mathscr{S}_{K}=\left\{\left(t, t^{d}\right) \mid t \in G F\left(q^{m}\right)\right\}$ for some $d=2^{i}$. Since $\mathscr{S}_{G_{\lambda}} \subset \mathscr{S}_{K}, m>1$. Since $\varphi\left(t, t^{d}\right)=\left(t^{d}, t^{2}\right) \in \mathscr{S}_{K}$, we get $t^{d^{2}}=t^{2}$ for all $t \in G F\left(q^{m}\right)$. Hence $m$ is odd and $K$ contains $(Z(U))_{\lambda_{m}}$. Conjugating by $N_{\lambda}$, we see that $K$ contains $\left(Z\left(U_{\rho}\right)\right)_{\lambda^{m}}$ for any nonabelian root subgroup $U_{\rho}$ of $U$. Hence for all $t \in G F\left(q^{m}\right), K$ contains

$$
\left[x_{0110}(t) x_{0210}\left(t^{d}\right), x_{1111}(1) x_{2211}(1)\right] \text {, }
$$

which, modulo terms in $\left(Z\left(U_{\rho}\right)\right)_{\lambda^{m}}$ for various nonabelian $U_{\rho}$, equals $x_{1221}(t) x_{2421}\left(t^{d}\right)$. Thus $K$ contains $\left(U_{\rho}\right)_{\lambda^{m}}$ for all abelian root subgroups $U_{\rho}$. Hence $K \supseteqq\left\langle\left(X_{1000} X_{0001}\right)_{\lambda}, N_{\lambda}\right\rangle \supseteqq\left\{h_{1000}(t) h_{0001}\left(t^{d}\right) \mid t \in G F\left(q^{m}\right)\right\}$. Conjugating $x_{0100}(1) x_{0010}(1) x_{0110}(1)\left(\in G_{\lambda}\right)$ by these element yields

$$
\left(X_{0100} X_{0010} X_{0110} X_{0210}\right)_{\lambda^{m}} \subseteq K
$$

Hence $K \supseteqq U_{\lambda^{m}}$, so $K \supseteqq G_{\lambda m}^{s}$.

If $\mathscr{S}_{K}$ contains an element of the form $(t, 0)$ or $(0, t)$ with $t \neq 0$, then since $\phi$ maps $\mathscr{S}_{K}$ to $\mathscr{S}_{K}, \mathscr{S}_{K} \supseteqq G F(q) \oplus G F(q)$. Hence $K$ contains $\left(Z\left(U_{\rho}\right)\right)_{\lambda^{2}}$ for all nonabelian root subgroups $U_{\rho}$ of $U$. From the commutator $\left[x_{0110}(t), x_{1111}(1)\right]$ we see that $K$ contains $\left(U_{\rho}\right)_{\lambda^{2}}$ for all abelian root subgroups $U_{\rho}$ of $U$. If $q>2$, we apply the argument of case 5 to the group generated by a nonabelian root group and its negative, and conclude that $\left(U_{\rho}\right)_{\lambda^{2}} \subseteq K$ for all nonabelian root groups $U_{\rho}$, whence $G_{\lambda^{2}}^{s} \cong K$. If $q=2$, a direct examination of $C_{2}(2)\left(\cong S_{6}\right.$, the symmetric group) shows that ${ }^{2} C_{2}(2)$ and a Sylow 2-center generate $C_{2}(2)$, whence $\left(U_{\rho}\right)_{\lambda^{2}} \subseteq K$ for all nonabelian root groups $U_{\rho}$, so again 


\section{$G_{\dot{\lambda}^{2}}^{s} \cong K$. This completes the proof of Lemma 2.5.}

(2.4) Proof. Second part. We continue with the assumptions given in (2.3). As a consequence of Lemma 2.5 we have a unique $\mu=\lambda^{r}$ such that $G_{\mu}^{s} \subseteq M$ and $U_{\mu} \in \operatorname{Syl}_{p}(M)$. Put $K=G_{\mu} \cap M$. In this sub-section we will show that $K=M$. Apart from the ${ }^{2} G_{2}$-case this will complete the proof of the theorem.

We use induction on the rank of $G$. The first step is when $G$ is of type $A_{1}$. Since $\mu \neq \sigma_{2}, \sigma_{3}$ we see from [6] that in this case $K=M$.

The induction will be applied to the components of semi-simple groups which occur in parabolic subgroups of $G$ and, when $p \neq 2$, in centralizers of involutions in $G$. Since such components may have the same rank as $G$ we perform the same rank as $G$ we perform the induction among groups of the same rank in the following order,

$$
A<(C, D, G)<(B, E)<F \text {. }
$$

This partial ordering insures that the induction procedure is valid when the above described subgroups hare the same rank as $G$.

To begin, we review some elementary facts. Let $\widetilde{S}$ be a connected, semi-simple, algebraic group and $\mu$ an endomorphism of $\widetilde{S}$ onto itself with $\widetilde{S}_{\mu}$ finite. Since $\mu$ must permute the components of $\widetilde{S}$ we have a unique decomposition $\widetilde{S}=\widetilde{F}_{1} \widetilde{F}_{2} \ldots$ where $\widetilde{F}_{i} \cap \widetilde{F}_{j} \subseteq$ $Z(\widetilde{S})$ for $i \neq j$ and each $\widetilde{F}_{i}$ has the form

$$
\widetilde{S}=\tilde{A} \mu(\tilde{A}) \cdots \mu^{n-1}(\tilde{A})
$$

with $\mu^{n}(\widetilde{A})=\widetilde{A}$ and $\widetilde{A}$ a component of $\widetilde{S}$.

For $\widetilde{X}$ one of $\widetilde{S}, \widetilde{F}, \widetilde{A}$ put $X=\widetilde{X} / Z(\widetilde{X})$ and note that $\mu$ is naturally defined on $S$ and $F$ and $\mu^{n}$ on $A$. It is easily seen that $F_{\mu}^{s} \cong A_{\mu^{n}}^{s}$ and that the images of $\widetilde{S}_{\mu}^{s}$ and $N_{\widetilde{S}}\left(\widetilde{S}_{\mu}^{s}\right)$ in $S$ are, using an obvious extension of Lemma 1.2, respectively $S_{\mu}^{s}$ and $S_{\mu}$.

The purpose of the next lemma is to extend the conclusion of Theorem 1 to the case where $G$ is replaced by a semi-simple group $\widetilde{S}$. This lemma is used in the proofs of Lemmas 2.8 and 2.9. In the situations there the assumption (i) below will hold because of our induction hypothesis.

Lemma 2.6. Let $\widetilde{S}$ be a connected, semi-simple, algebraic group and $\mu$ an endomorphism of $\widetilde{S}$ onto itself with $\widetilde{S}_{\mu}$ finite. For a component $\widetilde{A}$ of $\widetilde{S}$ put $A=\widetilde{A} / Z(\widetilde{A})$. Assume that

(i) For each component $\widetilde{A}$ of $\widetilde{S}$ the conclusion of Theorem 1 holds with $G$ replaced by $A$ and $\lambda$ replaced by $\mu^{n}$, where $n$ is the length of the r-orbit containing $\tilde{A}$. 
(ii) $\tilde{L}$ is a finite subgroup of $\widetilde{S}$ satisfying $\widetilde{S}_{\mu}^{s} \subseteq \widetilde{L}$ and $\left|\widetilde{L}: \widetilde{S}_{\mu}^{s}\right|_{p}=1$. Then $\tilde{L}$ normalizes $\widetilde{S}_{\mu}^{s}$.

Proof. Put $S=\widetilde{S} / Z(\widetilde{S})$ and $L=\tilde{L} Z(\widetilde{S}) / Z(\widetilde{S})$ then since $N_{\widetilde{S}}\left(\widetilde{S}_{\mu}^{s}\right) Z(\widetilde{S}) / Z(\widetilde{S})=S_{\mu}$ it suffices to show that $L \cong S_{\mu}$.

Suppose first that the components of $S$ form a single $\mu$-orbit. Thus $S=A \times B$ where $A$ is a component and $B=\mu(A) \times \cdots \times$ $\mu^{n-1}(A)$ and $\mu^{n}(A)=A$. If $n=1$ then $B=1$. Now $B L \cap A$ is finite and $B S_{\mu}^{s} \cap A=A_{\mu^{n}}^{s}$ and hence $\left|B L \cap A: A_{\mu^{n}}^{s}\right|_{p}=1$. By assumption

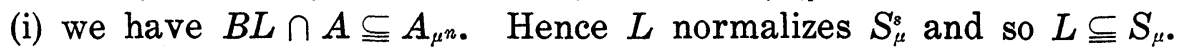

We now use induction on the number of $\mu$-orbits of components in $S$. Suppose $S=E \times F$ where $E, F$ are nontrivial products of $\mu$-orbits. Then $S_{\mu}=E_{\mu} \times F_{\mu}$ and $S_{\mu}^{s}=E_{\mu}^{s} \times F_{\mu}^{s}$. Again we have $E L \cap F$ finite and $E S_{\mu}^{s} \cap F=F_{\mu}^{s}$ and hence $\left|E L \cap F: F_{\mu}^{s}\right|_{p}=1$. By induction $E L \cap F \subseteq F_{\mu}$. Similarly $F L \cap E \subseteq E_{\mu}$. Hence $L \subseteq(E L \cap F) \times$ $(F L \cap E) \subseteq F_{\mu} \times F_{\mu}=S_{\mu}$.

Note. In the two situations where the above lemma is used assumption (i) fails to hold only if $A, \mu^{n}$ are one of the 3 exceptional cases described in (2.1). Furthermore $n=1$ except in one special occurrence in Lemma 2.8 with $G_{\mu}^{s}={ }^{2} F_{4}(2)$ and $\widetilde{S}$ of type $A_{1} \times A_{1}$. If $\widetilde{S}$ has an orbit $\widetilde{E}$ containing a component $\widetilde{A}$ such that $A, \mu^{n}$ do not satisfy assumption (i) we call this an exceptional orbit (and $\widetilde{E}=$ $\widetilde{A}$ except for one case). From the last step of the above proof we see that if $\widetilde{E}$ is an exceptional orbit the conclusion of the lemma still holds provided $F L \cap E$ normalizes $E_{\mu \cdot}^{s}$. Now $L \cap E \leqq F L \cap E$ and by inspection of the cases in (2.2) we conclude that if $L \cap E$ normalizes $E_{\mu}^{s}$ then $F L \cap E$ must also normalize $E_{\mu}^{s}$. We may conclude that if $\widetilde{E}$ is an exceptional orbit of $\widetilde{S}$ then the conclusion of the lemma still holds provided $\widetilde{L} \cap \widetilde{E}$ normalizes $\widetilde{E}_{\mu \text { e }}^{s}$

\section{LEMMA 2.7. $M \cap B=K \cap B$.}

Proof. Since $U_{\mu} \in \operatorname{Syl}_{p}(M)$ we have $M \cap U=K \cap U$ and hence $M \cap B=N_{M}\left(U_{\mu}\right)$, using Lemma 2.3. Let $g \in M \cap B$, since $B_{\mu}=H_{\mu} U_{\mu}$ we may suppose that $g=h z$ where $h \in H_{\mu}$ and $z \in Z(U)$. If $h \in M$ then $z \in Z(U) \cap M \subseteq U_{\mu}$ and so $g \in K$.

If $h \notin M$ we argue as follows. First suppose $Z(U)$ is 2-dimensional. In such a case it is is always true that $G_{\mu}=G_{\mu}^{s}$ and hence $H_{\mu} \subseteq M$. Thus we may suppose that $Z(U)$ is one-dimensional. Thus $Z(U)=$ $\left\langle x_{\theta}(t) \mid t \in k\right\rangle$ where $\theta$ is the root of maximal height in $\Sigma^{+}$. If $G$ is not of type $A_{1}$ or $C_{l}, l \geqq 2$, then $\theta$ is either a fundamental weight or for $A_{l}, l \geqq 2$, the sum of two distinct fundamental weights. This 
implies that there exists $h_{1} \in H \cap G_{\mu}^{s}$ such that $h_{1}(\theta)=h(\theta)$ and hence $\left[h_{1}^{-1} h, z\right]=1$ (here we identify $H$ with Hom $\left(\Gamma, k^{*}\right)$ ). Since $H \cap G_{\mu}^{s} \subseteq$ $H_{\mu} \cap M, h_{1}^{-1} h z \in M \cap B$ and since $h_{1}^{-1} h$ and $z$ have coprime orders $z \in$ $M \cap B$. Hence $z \in U_{\mu}$ and again $g \in K$.

If $G$ is of type $A_{1}$ we quote L. Dickson [6].

If $G$ is of type $C_{l}$ let $z=x_{\theta}(t)$ for some fixed $t \in k$, where $\theta=$ $\alpha_{1}+2 \alpha_{2}+\cdots+2 \alpha_{l}$. We may choose $h_{1} \in H \cap G_{\mu}^{s}$ such that if $h_{2}=$ $h_{1} h$ then, for some $s \in k^{*}$,

$$
h_{2}\left(\alpha_{1}\right)=s \quad h_{2}\left(\alpha_{2}\right)=\cdots=h_{2}\left(\alpha_{l}\right)=1 .
$$

Let $w_{i} \in W$ denote the reflection corresponding to $\alpha_{i} \in \Pi$. Put $n_{i}=$ $n_{w_{i}} \in N$ and $n=n_{2} \cdots n_{l}$. It is easily checked that $n h_{2} z n^{-1}=$ $h_{2} x_{\alpha_{1}}( \pm t) \in M \cap B$. Now $h_{2} x_{\alpha_{1}}( \pm t) h_{2} x_{\theta}(t)=h_{2}^{2} x_{\alpha_{1}}\left( \pm s^{-1} t\right) x_{\theta}(t)$ and since $h_{2}^{2} \in M$ therefore $x_{\alpha_{1}}\left( \pm s^{-1} t\right) x_{\theta}(t) \in M$. Since $M \cap U=U_{\mu}$ we have $z=$ $x_{\theta}(t) \in U_{\mu}$ and so $g \in K$.

Let $X$ be a subgroup of the finite group $Y$. Recall that $X$ is said to be strongly $p$-embedded in $Y$ if $\left|X \cap X^{y}\right|_{p}=1$ for all $y \in Y-X$. Using Sylow's theorems we see that $X$ is strongly $p$-embedded in $Y$ if and only if $N_{Y}(T) \subseteq X$ for all $1 \neq T \subseteq S$ where $S \in \operatorname{Syl}_{p}(X)$. The 'only if' part is clear. Conversely, take $y \in Y-X$ and assume $p|| X \cap X^{y} \mid$. Let $P \in \operatorname{Syl}_{p}\left(X \cap X^{y}\right)$. Then $N_{Y}(P) \subseteq X$, so that $P \in$ $\operatorname{Syl}_{p}\left(X^{y}\right)$. Therefore $P, P^{y^{-1}} \in \operatorname{Syl}_{p}(X) \subseteq \operatorname{Syl}_{p}(Y)$ as well. Choose $x \in$ $X$ with $P=P^{y x}$. Thus $y x \in N_{Y}(P) \subseteq X$, so that $y \in X$, as required.

Lemma 2.8. $K$ is strongly p-embedded in $M$.

Proof. Let $1 \neq T_{\mu}$ then a theorem of A. Borel and J. Tits [4] implies the existence of a parabolic subgroup $P \subset G$ such that $P$ is fixed by $\mu$ and $N_{G}(T) \subseteq P$. Without restriction we may suppose $B \subseteq P$. If $P \subseteq B$ by Lemma 2.7 we have $N_{\mu}(T) \subseteq K$. If $P \neq B$ let $R=$ radical of $P$ and put $\widetilde{S}=P / R$. $\widetilde{S}$ is a connected, semi-simple, algebraic group and $\mu$ acts naturally on it. Put $\widetilde{M}=(M \cap P) R / R$, $\widetilde{K}=(K \cap P) R / R$ then $\widetilde{S}_{\mu}^{s} \cong \widetilde{K} \subseteq N_{\widetilde{S}}\left(\widetilde{S}_{\mu}^{s}\right)$. If $\widetilde{S}$ has no exceptional orbits Lemma 2.6 says that $\widetilde{M}$ normalizes $\widetilde{K}$. By Lemma 2.7, since $R \subseteq B$, we have $M \cap R=K \cap R$. Hence $M \cap P$ normalizes $K \cap P$ and so, again using Lemma 2.7, $M \cap P=(K \cap P) N_{M \cap P}\left(U_{\mu}\right)=K \cap P$. Hence $K$ is strongly $p$-embedded in $M$.

Suppose next that $\widetilde{A}$ is an exceptional orbit in $\widetilde{S}$. By the note following Lemma 2.6 we must show that $\widetilde{M} \cap \widetilde{A}$ normalizes $\widetilde{K} \cap \widetilde{A}$.

Let $V$ be the unipotent radical of $P$ and put $W=V / V^{\prime}$. Let $W_{\mu}$ be the image $V_{\mu}$ in $W$. Since $V^{\prime}$ is closed and connected an argument similar to that in Lemma 2.3 shows that $W_{\mu}$ is just the fixed points of the endomorphism $v V^{\prime} \rightarrow \mu(v) V^{\prime}, v \in V$, of $W$. 
Now $V_{\mu}=K \cap V=M \cap V$ so $\tilde{M} \cap \tilde{A}$ normalizes $W_{\mu}$. Hence for all $k \in \tilde{M} \cap \tilde{A}, k^{-1} \mu(k)$ centralizes $W_{\mu}$. Our aim is to show that $C_{\tilde{A}}\left(W_{\mu}\right) \subseteq$ $Z(\tilde{A})$. This will immediately give $\tilde{M} \cap \widetilde{A} \cong N_{\widetilde{A}}\left(\widetilde{A}_{\mu}\right)$ and since $N_{\widetilde{A}}\left(\widetilde{A}_{\mu}\right)=$ $N_{\tilde{A}}(\widetilde{K} \cap \widetilde{A})$ we are done.

To compute $C_{\tilde{A}}\left(W_{\mu}\right)$ we may suppose $P$ is maximal, subject to $\mu(P)=P$. Let $\Delta$ be a proper subset of $\Pi$ such that $\Pi-\Delta$ contains no proper $\mu$-invariant subset (note that $\mu$ permutes $\Pi$ ) then

$$
\left.P=\left\langle x_{\gamma}(t)\right| \gamma \in \Sigma^{+} \text {or }-\gamma \in \Delta, t \in k\right\rangle
$$

and the choice of $\Delta$ is further restricted by requiring $\tilde{A}$ to be a component of $\widetilde{S}=P / R$. The possible cases are easily listed: except when $G_{\mu}^{s}$ is ${ }^{2} A_{l}(l=$ odd $),{ }^{3} D_{4},{ }^{2} F_{4} . \quad \Pi-\Delta$ is a single root, say $\alpha$, and $\widetilde{A}$ is the image modulo $R$ of $\left\langle x_{\beta}(t), x_{-\beta}(t) \mid t \in k\right\rangle$ some $\beta \in \Delta$. In this case an $\tilde{A}$-invariant, $\mu$-invariant submodule $W_{1}$ of $W$ has basis

$$
\left\{x_{\gamma}(1) \mid \gamma=\alpha, \alpha+\beta, \alpha+2 \beta, \cdots\right\} \bmod V^{\prime} \text {. }
$$

It is easily seen that $C_{\tilde{A}}\left(\left(W_{1}\right)_{\mu}\right) \subseteq Z(\widetilde{A})$.

When $|\Pi-\Delta| \geqq 2, \widetilde{A}$ is again of type $A_{1}$ except for the ${ }^{2} F_{4}$ case when $\widetilde{A}$ is either of types $A_{1} \times A_{1}$ or $C_{2}$. Again a suitable $\tilde{A}$ - and $\mu$-invariant sub-module $W_{1} \subseteq W$ is easily found such that $C_{\tilde{A}}\left(\left(W_{1}\right)_{\mu}\right) \subseteq$ $Z(\tilde{A})$. For example in the ${ }^{2} F_{4}$ case with $\tilde{A}$ the image modulo $R$ of $\left\langle x_{\beta}(t) \mid \beta= \pm \alpha_{1}, \pm \alpha_{4}, t \in k\right\rangle$ let $W_{1}$ have basis

$$
\left\{x_{\gamma}(1) \mid \gamma=\alpha_{2}, \alpha_{3}, \alpha_{1}+\alpha_{2}, \alpha_{3}+\alpha_{4}\right\}
$$

then $\left(W_{1}\right)_{\mu}$ has basis $\left\{x_{\alpha_{2}}(1) x_{\alpha_{3}}(1), x_{\alpha_{1}+\alpha_{2}}(1) x_{\alpha_{3}+\alpha_{4}}(1)\right\}$.

Lemma 2.9. $K$ is strongly 2-embedded in $M$.

Proof. By Lemma 2.8 we may suppose $p \neq 2$. If the lemma is false then there exists a $t \in \operatorname{Inv}\left(K \cap K^{m}\right)$ for some $m \in M-K$. Now $C_{G}(t)$ contains a unique, maximal, semi-simple, connected algebraic $\widetilde{S}$, [18]. Since we may suppose $G$ is not of type $A_{1}, \widetilde{S} \neq 1$. Since $\mu(t)=t, \mu$ normalizes $\widetilde{S}$ and hence $\widetilde{S}_{\mu}^{s} \subseteq \widetilde{S} \cap K \subseteq \widetilde{S} \cap M$.

Since all $p$-elements of $C_{G}(t)$ lie in $\widetilde{S}$ we have $\left|\widetilde{S} \cap K^{m}\right|_{p} \neq 1$. By Lemma $2.8\left|K \cap K^{m}\right|_{p}=1$ and hence $O^{p^{\prime}}(\widetilde{S} \cap M) \nsubseteq \widetilde{S} \cap K$. However if $\widetilde{S}$ contains no exceptional orbits Lemma 2.6 implies $O^{p^{\prime}}(\widetilde{S} \cap M) \cong$ $\widetilde{S} \cap K$, contradiction.

If $\tilde{A}$ is an exceptional orbit of $\widetilde{S}$ then $\tilde{A}$ is of type $A_{1}$ and $p=$ 3. If $\tilde{A} \cap M$ does not normalize $\tilde{A} \cap K$ then from the list of exceptional cases in (2.2) we see that $\tilde{A} \cap K$ is not strongly 3-embedded in $\widetilde{A} \cap M$. But then $K$ is not strongly 3 -embedded in $M$, contradicting Lemma 2.8 . 
LEMMA 2.10. $K=M$.

Proof. Suppose $K \neq M$, by Lemma 2.9 and a theorem of $\mathrm{H}$. Bender [2] either the Sylow 2-subgroup of $K$ is cyclic or quaternion or $K$ is solvable. Using ref. [8], [12] and a theorem of Burnside we see that $K$ has no non-abelian simple subgroups. Since $K$ contains $\left[G_{\mu}^{s}, G_{\mu}^{s}\right]$ it follows that $G_{\mu}$ is ${ }^{2} A_{2}(2)$.

Let $t \in \operatorname{Inv} K$ then $K=O_{2^{\prime}}(K) C_{K}(t)$ and $O_{2^{\prime}}\left(C_{K}(t)\right)=1$. By Lemma $2.9 C_{K}(t)=C_{M}(t)$ and so by [12], $M=O_{2^{\prime}}(M) C_{K}(t)$. Then $O_{2^{\prime}}(K) \subseteq$ $O_{2^{\prime}}(M)$ and $C_{O_{2^{\prime}(M)}}(t) \cong O_{2^{\prime}}\left(C_{K}(t)\right)=1$ so $O_{2^{\prime}}(M)$ is abelian. Hence $M \subseteq$ $N_{G}\left(O_{2^{\prime}}(K)\right)$ and now a direct calculation yields $N_{G}\left(O_{2^{\prime}}(K)\right)=G_{\mu}$. So $K=M$, a contradiction.

(2.5) Proof. ${ }^{2} G_{2}$-case. In this subsection $G$ is of type $G_{2}$ and $\lambda={ }^{2} \sigma_{q}$ where $q=3 q_{0}^{2}, q_{0}=3^{f}$. For this case we give a direct proof of the theorem by analyzing the structure of $C_{M}(j)$ where $j$ is an involution in $G_{\lambda}$.

Proof. We let $\mu$ be the highest power of $\lambda$ such that $G_{\mu} \subseteq M$, and show that $M=G_{\mu}$. Without loss, we may assume $\mu=\lambda$, since the various powers of $\lambda$ are ${ }^{2} \sigma_{q m}$ and $\sigma_{q m}$, and the $\sigma_{q m}$-case has already been done.

We take $\Pi=\{\alpha, \beta\}$, with $\alpha$ long and choose notation so the commutator formulas are as in [15]. Let $j$ be the element of $H$ such that $j(\alpha)=j(\beta)=-1$ and let $C=C_{G}(j)$. Thus $\operatorname{ker} j \cap \Sigma^{+}=$ $\{\alpha+\beta, \alpha+3 \beta\}$, so $C=L_{1} L_{2}$, where $L_{1}=\left\langle X_{\alpha+\beta}, X_{-\alpha-\beta}\right\rangle, L_{2}=\left\langle X_{\alpha+3 \beta}\right.$, $\left.X_{-\alpha-3 \beta}\right\rangle,\left[L_{1}, L_{2}\right]=1, L_{1} \cap L_{2}=Z(C)=\langle j\rangle$, and each $L_{i}$ is isomorphic to $S L_{2}(k)$. Clearly $j \in G_{\lambda}$. For any subgroup $J$ of $G$ let $C_{J}=C_{J}(j)$.

Put $x_{+}^{*}(t)=x_{\alpha+\beta}(t) x_{\alpha+3 \beta}\left(t^{3 q_{0}}\right)$ and define $x_{-}^{*}(t)$ similarly, and let $L=$ $\left\langle x_{+}^{*}(t), x_{-}^{*}(t) \mid t \in G F(q)\right\rangle$. Then $L \cong P S L_{2}(q)$ and $C_{G_{\lambda}}=L \times\langle j\rangle$.

Suppose $C_{M} \subseteq N_{C}\left(C_{G_{\lambda}}\right)$. Let $T_{G_{\lambda}}, T_{M}$, and $T_{N}$ be Sylow 2-subgroups of $C_{G_{\lambda}}, C_{M}$, and $N_{C}\left(C_{G_{\lambda}}\right)$, respectively, such that $T_{G_{\lambda}} \subseteq T_{M} \subseteq T_{N}$. An easy computation shows $N_{C}\left(C_{G_{\lambda}}\right)=T_{N} C_{G_{\lambda}}, T_{N}$ is nonabelian of order 16 , $T_{G_{\lambda}}$ is elementary abelian of order 8 , and $\left|N_{G_{\lambda}}\left(T_{G_{\lambda}}\right) / C_{G_{\lambda}}\left(T_{G_{\lambda}}\right)\right|=21$. If $T_{M}=T_{N}$, then $\left|N_{M}\left(T_{G_{\lambda}}\right) / C_{M}\left(T_{G_{\lambda}}\right)\right|=42$, which is absurd since $G L(3,2)$ has no subgroups of order 42 . Thus $T_{M} \subset T_{N}$, so $C_{M}=T_{M} C_{G_{\lambda}}=C_{G_{\lambda}}$. By a theorem of Walter [28], $|M|=\left|G_{\lambda}\right|$, so $M=G_{\lambda}$, as required. Thus, we may assume $C_{M} \nsubseteq N_{C}\left(C_{G_{\lambda}}\right)$.

Let $\bar{C}=C /\langle j\rangle$, and for any $A \subseteq C$ write $\bar{A}$ for $A\langle j\rangle /\langle j\rangle$. Then $\bar{C}=\bar{L}_{1} \times \bar{L}_{2}, \bar{L}_{i}$ isomorphic to $P S L_{2}(k)$. Let $\pi_{i}, i=1,2$, be the projection $\bar{C}$ on $\bar{L}_{i}$.

Suppose $\pi_{1}(\bar{L}) \subseteq \bar{C}_{M}$. Since $\bar{L} \cong \bar{C}_{M}$, also $\pi_{2}(\bar{L}) \subseteq \bar{C}_{M}$. Since $j \in$ $C_{M}$, we get $x_{\rho}(t) \in M$ for $\rho= \pm(\alpha+\beta), \pm(\alpha+3 \beta)$, and all $t \in G F(q)$. In particular, $n_{\alpha+\beta}(1) \in M$. Now $U_{\lambda}$ contains an element 


$$
x=x_{\alpha}(1) x_{\beta}(1) \cdots,
$$

so $M$ contains $\left[x, x_{\alpha+3 \beta}(t)\right]=x_{2 \alpha+3 \beta}( \pm t)$ for all $t \in G F(q)$. Conjugating by $N_{\lambda}$, we find $x_{-2 \alpha-3 \beta}(t) \in M$ for all $t \in G F(q)$. Hence $M$ contains $n_{2 \alpha+3 \beta}(1)$. Since $W=\left\langle w_{\alpha+\beta}, w_{2 \alpha+3 \beta}\right\rangle, M$ covers $N / H$. As $\left\langle\left(X_{\alpha+\beta}\right)_{\lambda^{2}}\right.$, $\left.\left(X_{\alpha+3 \beta}\right)_{\lambda^{2}}\right\rangle \subseteq M$, it follows that $G_{\lambda^{2}} \leqq M$. Thus, we may assume $\pi_{1}(\bar{L}) \nsubseteq \bar{C}_{M}$, and similarly, $\pi_{2}(\bar{L}) \nsubseteq \bar{C}_{M}$.

Suppose next that $\pi_{1}\left(\bar{C}_{M}\right)$ is not solvable. Now $\pi_{1}(\bar{L})=\left(\bar{L}_{1}\right)_{\lambda}^{s} 2$, so either $\pi_{1}\left(\bar{C}_{M}\right)^{s}=\left(\bar{L}_{1}\right)_{\lambda_{2}^{2}}^{s}$ for some $m$, or else $q=3$ and $\pi_{1}\left(\bar{C}_{M}\right) \cong$ $A_{5}$, the alternating group. To see this observe that since $\pi_{1}\left(\bar{C}_{M}\right)$ is finite its inverse image in $L_{1}$ is a finite subgroup of $S L_{2}(k)$ and so is conjugate in $G L_{2}(k)$ to a subgroup of $S L_{2}\left(3^{f}\right)$ for some $f$. Hence for purposes of identifying $\pi_{1}\left(\bar{C}_{M}\right)$ up to isomorphism, we may assume it lies in $S L_{2}\left(3^{f}\right)$. If $3^{2} \nmid\left|\pi_{1}\left(\bar{C}_{M}\right)\right|$, the argument of Lemma 2.4 shows that $\pi_{1}\left(\bar{C}_{M}\right) \cong\left(\bar{L}_{1}\right)_{\lambda^{2 n}}$ for some $n$ and Dickson's results [6] may be used. While if $3^{2} \nmid\left|\pi_{1}\left(\bar{C}_{M}\right)\right|$, these results imply $\pi_{1}\left(\bar{C}_{M}\right) \cong A_{5}$.

If $\pi_{1}\left(\bar{C}_{M}\right) \cong A_{5}$, then $\bar{C} M \cap \bar{L}_{1} \triangleleft \pi_{1}\left(\bar{C}_{M}\right)$ and $\pi_{1}(\bar{L}) \varsubsetneqq \bar{C}_{M}$ imply $\bar{C}_{M} \cap$ $\bar{L}_{1}=1$. Hence $\pi_{2}\left(\bar{C}_{M}\right) / \bar{C}_{M} \cap \bar{L}_{2} \cong A_{5}$, so $\pi_{2}\left(\bar{C}_{M}\right)$ is nonsolvable. Applying the above argument to $\pi_{2}\left(\bar{C}_{M}\right)$ yields $\pi_{2}\left(\bar{C}_{M}\right) \cong A_{5}$, hence $\bar{C}_{M} \cong A_{5}$, so $C_{M} \cong Z_{2} \times A_{5}$. Since $M$ contains $G_{\lambda} \cong{ }^{2} G_{2}(3)$, all involutions in $C_{M}$ are $M$-conjugate in this case, so by a theorem of Janko [19], $3^{2} \nmid|M|$, which is absurd as $G_{\lambda} \subseteq M$.

Hence, $\pi_{1}\left(\bar{C}_{M}\right)^{s}=\left(\bar{L}_{1}\right)_{\lambda_{2}^{2 m}}^{s}$. Since we are assuming that $\pi_{1}\left(\bar{C}_{M}\right)$ is not solvable this group is simple, so as in the $A_{5}$ case we get $\pi_{2}\left(\bar{C}_{M}\right)^{s}=$ $\left(\bar{L}_{2}\right)_{\lambda}^{s} 2 m, \bar{C}_{M}^{s} \cap \bar{L}_{1}=\bar{C}_{M}^{s} \cap \bar{L}_{2}=1$. If $m=1$, then $\bar{L} \subseteq \bar{C}_{M}$ implies $\bar{L}=$ $\bar{C}_{M}^{s}$, so $\bar{C}_{M} \subseteq N_{G}(\bar{L})$, contrary to what was shown above. Hence $m>$ 1. Now $\bar{C}_{M}^{s}$ is defined by an isomorphism between the $\pi_{i}\left(\bar{C}_{M}\right)^{s}$, which restricts on $\pi_{i}(\bar{L})$ to $x_{ \pm(\alpha+\beta)}(t) \leftrightarrow x_{ \pm(\alpha+3 \beta)}\left(t^{3 q_{0}}\right)$. From the well-known classification of automorphisms of $P S L_{2}$ there exists $d=3^{i}$ such that $\bar{C}_{M}^{s}=\left\langle\bar{x}_{ \pm}^{*}(t) \mid t \in G F\left(q^{m}\right)\right\rangle$, where we define $x_{+}^{*}(t)=x_{(\alpha+\beta)}(t) x_{(\alpha+3 \beta)}\left(t^{d}\right)$ and $x_{-}^{*}$ is defined similarly. (This extends previous notation; $t^{d}=t^{3 q_{0}}$ for $t \in G F(q)$.) Hence $C_{M}^{s}=\left\langle x_{ \pm}^{*}(t) \mid t \in G F\left(q^{m}\right)\right\rangle$. Set $h^{*}(t)=h_{\alpha+\beta}(t) h_{\alpha+3 \beta}\left(t^{d}\right)$. Since $\left[L_{1}, L_{2}\right]=1, C_{M}^{s}$ contains $h^{*}(t)$ for all $t \in G F\left(q^{m}\right)$.

Let $x, y$ and $z$ be elements of $G_{\lambda}$ of the form $x=x_{\alpha}(1) x_{\beta}(1) \cdots, y=$ $x_{\alpha+\beta}(1) x_{\alpha+3 \beta}(1) \cdots, z=x_{\alpha+2 \beta}(1) x_{2 \alpha+3 \beta}(1)$, then for any $t, u \in G F\left(q^{m}\right)^{x}, M$ contains the following elements:

$$
\begin{gathered}
x^{h^{*}(t)}=x_{\alpha}\left(t^{3-d}\right) x_{\beta}\left(t^{d-1}\right) \cdots, y^{h^{*}(u)}=x_{\alpha+\beta}\left(u^{2}\right) x_{\alpha+3 \beta}\left(u^{2 d}\right) \cdots \\
{\left[x^{h *(t)}, y^{h *(u)}\right]=x_{\alpha+2 \beta}\left(t^{d-1} u^{2}\right) x_{2 \alpha+3 \beta}\left(t^{3-d} u^{2 d}\right) .}
\end{gathered}
$$

Since every element of $G F\left(q^{m}\right)$ is a sum of square, $M$ contains

$$
x_{\alpha+2 \beta}\left(t^{d-1} u\right) x_{2 \alpha+3 \beta}\left(t^{3-d} u^{d}\right) \text {. }
$$

Replacing $u$ by $u t^{d-1}$ and $t$ by 1 in (3), and multiplying the resulting 
element by the inverse of (3), we get

$$
x_{2 \alpha+3 \beta}\left(\left(t^{3-d}-t^{d^{2}-d}\right) u^{d}\right) \in M \text {. }
$$

Also, $M$ contains

$$
\left[x^{h *(t)}, x\right]=x_{\alpha+\beta}\left(t^{3-d}-t^{d-1}\right) x_{\alpha+3 \beta}\left(t^{3 d-3}-t^{3-d}\right) \cdots .
$$

Suppose $t_{0}^{d^{2}} \neq t_{0}^{3}$ for some $t_{0} \in G F\left(q^{m}\right)$. From (4), $x_{2 \alpha+3 \beta}(t) \in M$ for all $t \in G F\left(q^{m}\right)$, and then from (3), $x_{\alpha+2 \beta}(t) \in M$ for all $t$. By. (1),

$$
x_{\alpha+\beta}(u) x_{\alpha+3 \beta}\left(u^{d}\right) \in M,
$$

and by (5), $x_{\alpha+\beta}\left(t^{3-d}-t^{d-1}\right) x_{\alpha+3 \beta}\left(t^{3 d-3}-t^{3-d}\right) \in M$. Substituting $t^{3-d}-t^{d-1}$ for $u$ and multiplying by the inverse of this last element,

$$
x_{\alpha+3 \beta}\left(t^{3 d-d^{2}}-t^{d^{2-d}}-t^{3 d-3}+t^{3-d}\right) \in M
$$

for all $t \in G F\left(q^{m}\right)$. Since $\bar{C}_{M}^{s} \cap \bar{L}_{2}=1$, the expression in parentheses vanishes identically. This yields

$$
\left(t^{3}-t^{d^{2}}\right)\left(t^{-d^{2}-3+3 d}+t^{-d}\right)=0
$$

for all $t \in G F\left(q^{m}\right)^{x}$. On the other hand, since $M$ contains $\left(X_{\alpha+2 \beta}\right)_{\lambda^{2 m}}$, $\left(X_{2 \alpha+3 \beta}\right)_{\lambda ₹ m}$, and an elment of $N_{G}(H)$ taking all roots to their negatives, $M$ contains $\hat{h}(t, u)=h_{\alpha+2 \beta}(t) h_{2 \alpha+\beta}(u)$ for all $t, u \in G F\left(q^{m}\right)^{x}$, so contains $y^{\hat{h}(t, u)}=x_{\alpha+\beta}\left(t^{3} u\right) x_{\alpha+3 \beta}\left(t u^{3}\right) \cdots$, hence contains $x_{\alpha+\beta}\left(t^{3} u\right) x_{\alpha+3 \beta}\left(t u^{3}\right)$. Since $\bar{C}_{M}^{s} \cap \bar{L}_{i}=1, i=1,2$, it follows that $t u^{3}=\left(t^{3} u\right)^{d}$ for all $t, u \in G F\left(q^{m}\right)$. Hence $u^{d}=u^{3}$ (take $\left.t=1\right)$ and $t^{3 d}=t($ take $u=1)$. Therefore $t^{d}=t^{3}$ and $t^{9}=t$ for all $t \in G F\left(q^{m}\right)$, so $q=3$ and $m=2$. For any $t \in$ $G F(9)-G F(3)$, we get $t^{d^{2}} \neq t^{3}$, and so by (6), $t^{d^{2-4 d+3}}=-1$. But the left side is $t^{9-12+3}=1$, contradiction.

Hence $t^{d^{2}}=t^{3}$ for all $t \in G F\left(q^{m}\right)$. This implies that $m$ is odd, and $C_{M}^{s}=C_{\lambda^{2 m}}$. Hence $M \cap G_{\lambda^{2 m}} \supseteqq\left\langle C_{\lambda^{2 m}}, G_{\lambda}\right\rangle \supset C_{\lambda^{2 m}}$. It follows from Walter's theorem [28] (applied to $M \cap G_{\lambda^{2 m}}$ ) that $\left|M \cap G_{\lambda^{2 m}}\right|=$ $\left|G_{\lambda^{2 m}}\right|$, i.e., $M \supseteq G_{\lambda^{2 m}}$, as required. Hence we may assume $\pi_{1}\left(\bar{C}_{M}\right)$ is solvable, and similarly that $\pi_{2}\left(\bar{C}_{M}\right)$ is solvable. In particular, $q=3$.

It follows from Dickson's results [6] that $\pi_{i}\left(\bar{C}_{M}\right) \cong N_{\bar{L}_{i}}(\bar{L}) \cong S_{4}$, the symmetric group for $i=1,2$. If 9|| $\bar{C}_{M} \mid$, it follows easily that $\pi_{1}(\bar{L}) \times \pi_{2}(\bar{L}) \subseteq \bar{C}_{M}$, contrary to what was shown above. Thus $\bar{C}_{M}$ has Sylow 3-subgroups of order 3. Since $\bar{C}_{M} \nsubseteq N_{\bar{C}}\left(\bar{C}_{G_{2}}\right), C_{M}$ must be an extension of the central product $Q_{8} * Q_{8}$ by either a group of order 3 or the symmetric group $S_{3}$. Let by a Sylow 2-subgroup of $C_{M}$. It is easily verified that $Z(T)=\langle j\rangle$. Hence $T$ is a Sylow 2-subgroup of $M$. Since $\left\langle j^{M}\right\rangle \supseteqq\left(G_{\lambda}\right)^{\prime}$, which is perfect, $O_{2}(M)=1$. Now $T_{\lambda}$ is elementary of order 8 , and all its nonidentity elements are conjugate in $M$ (indeed in $G_{2}$ ). Since $j \in T_{\lambda}$ and $O_{2^{\prime}}\left(C_{M}(j)\right)=1$, it follows that $O_{2^{\prime}}(M) \subseteq\left\langle O_{2^{\prime}}\left(C_{M}(i)\right) \mid i \in T_{:}^{\sharp}\right\rangle=1$. Let $M_{0}$ be a minimal normal subgroup 
of $M$. Thus $M_{0}$ is the direct product of isomorphic nonabelian simple groups. By [8], [12] and and a theorem of Burnside, each simple factor has 2-rank at least 2. However, one sees easily that $T$ has 2-rank 3. Hence, $M_{0}$ is simple. From the structure of $T$, we see that $T_{\lambda}=C_{T}\left(T_{\lambda}\right)$, and $\left|N_{T}\left(T_{\lambda}\right) / T_{\lambda}\right| \geqq 4$. On the other hand, since $\langle j\rangle=$ $Z(T), j \in M_{0}$, and so $\left(G_{\lambda}\right)^{\prime}=\left\langle j^{M}\right\rangle \subseteq M_{0}$, so $\left|N_{M_{0}}\left(T_{\lambda}\right) / T_{\lambda}\right|$ is divisible by 7. Since $N_{M_{0}}\left(T_{\lambda}\right) / T_{\lambda} \triangleleft N_{M}\left(T_{\lambda}\right) / T_{\lambda}$, a subgroup of $G L_{3}(2)$, it follows that $N_{M_{0}}\left(T_{\lambda}\right) / T_{\lambda}=N_{M}\left(T_{\lambda}\right) / T_{\lambda} \cong G L_{3}(2)$. In particular, $|T| \geqq 2^{6}$, so $|T|=2^{6}$, and also $T \subseteq M_{0}$. Hence $M_{0} \supseteqq T\left[T, C_{M}\right]=C_{M}$. By the the Frattini argument, $M=M_{0} N_{M}(T) \subseteq M_{0} C_{M}=M_{0}$, so $M=M_{0}$ is simple.

Quoting the classification of finite simple groups in which the centralizer of an involution (in the centre of Sylow 2-subgroups) is isomorphic to $C_{M}$, we find that the only such group which in addition has a subgroup isomorphic to $G_{\lambda}$ is the alternating group $A_{9}$ (see, for example [14]). Hence $M \cong A_{9}$.

Let $S$ be a Sylow 3-subgroup of $M$ containg $U_{\lambda}$. Then $|S|=3^{4}$, so $U_{\lambda} \triangleleft S$, i.e., $S \subseteq N_{G}\left(U_{\lambda}\right)$. By Lemma 1.1, $S \subseteq B$, so $S \subseteq U$. Let $U^{\prime}=$ $X_{\alpha+\beta} X_{\alpha+3 \beta} X_{\alpha+2 \beta} X_{2 \alpha+3 \beta}$. Now $S$ is the wreath product $Z_{3}<Z_{3}$. It follows easily that $S^{\prime}=U_{\lambda} \cap U^{\prime}=\left\langle x_{\alpha+\beta}(1) x_{\alpha+3 \beta}(1), x_{\alpha+2 \beta}(1) x_{2 \alpha+3 \beta}(1)\right\rangle$, and also that $S$ is generated by $U_{\lambda}$ and an element $z \in C_{U}\left(S^{\prime}\right)$ of order 3. The only such $z$ lie in $U^{\prime}$, so $S=U_{\lambda}\left(S \cap U^{\prime}\right)$. Hence $\left|S: S \cap U^{\prime}\right|=3$. Let $U^{2}=Z(U)=X_{\alpha+2 \beta} X_{2 \alpha+3 \beta}$. Then $U^{\prime} / U^{2}=Z\left(U / U^{2}\right)$, so $S \cap U^{\prime} / S \cap U^{2} \subseteq$ $Z\left(S / S \cap U^{2}\right)$, so $S / S \cap U^{2}$ is abelian. Hence $S^{\prime} \subseteq S \cap U^{2} \subseteq Z(S)$, contradiction. This completes the proof.

\section{Theorem 2 .}

(3.1) Statement of results. As in previous sections $G$ denotes a simple algebraic group over an algebraically closed field $k$ of characteristic $p \neq 0$.

We wish to examine certain $\eta \in$ Aut $\left(G_{\mu}\right)$ and determine the subgroups of $G_{\mu}$ lying above $C_{G_{\mu}}(\eta)$. We cannot restrict ourselves to $\eta$ induced on $G_{\mu}$ by an element of the form $g \cdot \lambda$, where $\lambda^{n}=\mu, 0<n \in$ $Z, g \in G_{\mu \cdot}$. For example, let $G=A_{l}(k), l \geqq 2, \mu={ }^{2} \sigma_{q}$. The "field" (or "graph") automorphism $\eta$ of $O^{p^{\prime}}\left(G_{\mu}\right)={ }^{2} A_{l}(q) \cong P S U(l+1, q)$ does not have the above shape. Indeed, it is induced on $G_{\mu}$ by $\lambda \in \operatorname{Aut}(G)$, $\lambda=\sigma_{q}$. Thus, to examine questions of this type, we must consider pairs of commuting endomorphisms $\lambda, \mu$ of $G$ with $G_{\lambda}$ and $G_{\mu}$ finite. Then some power of $\lambda$ centralizes $G_{\mu}$. We may suppose that $\mu, \lambda$ are in standard form (see 1.2) and put $G_{\mu, \lambda}=G_{\mu} \cap G_{\lambda}$.

THEOREM 2. Let $G$ be as described above. Let $r>1$ be an integer and $\lambda=\sigma_{q}, \mu={ }^{s} \sigma_{q^{r / s}}$ where $G$ possesses a graph automorphism of order $s \in\{2,3\}$ and $s$ divides $r$. 
Let $M$ be a group, $O^{p^{\prime}}\left(G_{\lambda, \mu}\right) \leqq M \leqq G_{\mu}$. Then precisely one of the following holds if $r$ is a prime (i.e., $r=s$ )

(1) $G_{\lambda, \mu} \cong C_{n}\left(2^{m}\right), G_{\mu} \cong{ }^{2} A_{2 n}\left(2^{m}\right), O^{2^{\prime}}(M) \cong{ }^{2} A_{2 n-1}\left(2^{m}\right), M / O^{2^{\prime}}(M)$ is cyclic of order dividing $2^{m}+1, n \geqq 2$.

(2) $M \leqq G_{\lambda, \mu}$

(3) $O^{p^{\prime}}\left(G_{\mu}\right) \leqq M$

(4) $p=2, G_{\lambda, \mu} \cong{ }^{2} C_{2}(2), G_{\mu} \cong{ }^{2} C_{2}\left(2^{r}\right) ; M$ lies in a a unique maximal subgroup $M_{0}$ which is a Frobenius group of order $4\left(2^{r} \pm 2^{(r+1) / 2}+1\right)$ and $G_{\mu} \cong{ }^{2} C_{2}\left(2^{r}\right)$ for odd $r \geqq 5$.

( 5 ) $\quad p=3, G_{\lambda, \mu} \cong P G L(2,3), G_{\mu} \cong{ }^{2} A_{2}(3) \cong U_{3}(3), G_{\lambda, \mu}<M<G_{\mu}, M \cong$ $\operatorname{PSL}(2,7)$,

(6) $p=5, G_{\lambda, \mu} \cong P G L(2,5), O^{5^{\prime}}\left(G_{\mu}\right) \cong{ }^{2} A_{2}(5) \cong U_{3}(5), G_{\lambda, \mu}<M_{i}<$ $O^{5^{\prime}}\left(G_{\mu}\right), i=1,2, M_{1} \cong A_{7}, M_{2} \cong M_{10}$.

Furthermore, if $r$ is not assumed to be prime, but $|M|_{p}=\left|G_{\lambda, \mu}\right|_{p}$, then $(x)$ holds, for some $2 \leqq x \leqq 6$.

We wish to emphasize the point that we have not fully examined the question: if $G_{\mu}$ is a finite group of Lie type and $\eta$ is a noninner automorphism, what are the subgroups of $G_{\mu}$ lying above $C_{G_{\nu}}(\eta)$ ? We have examined only the case where $\eta$ is induced on $G_{\mu}$ by $\lambda$, an endomorphism of $G$ with $\lambda^{r}=\mu$ or $\lambda=\sigma_{q} r$ and $\mu={ }^{s} \sigma_{q^{r / s}}$. For instance, letting $\lambda^{*}$ be the image of one of the above $\lambda$ in $\operatorname{Aut}\left(G_{\mu}\right)$, there may be an $\eta$ in the coset $\operatorname{Inn}\left(G_{\mu}\right) \cdot \lambda^{*}$ such that $|\eta|=\left|\lambda^{*}\right|$, yet $\eta$ and $\lambda^{*}$ are not conjugate in Aut $\left(G_{\mu}\right)$ or even $\left(G_{\mu}\right)_{\eta} \not \equiv\left(G_{\mu}\right)_{\lambda^{*}}$.

In proving the above result we may apply Theorem 1 wherever $\langle\lambda, \mu\rangle$ is a cyclic group; for then $\lambda$ may be replaced by a generator of $\langle\lambda, \mu\rangle$.

(3.2) An example. As an illustration of where our results do not apply we give the following example, for which we thank J. E. McLaughlin.

Take $G$ to have type $A_{3}, \mu={ }^{2} \sigma_{3}, \lambda=\sigma_{3}$. Then $L=O^{3^{\prime}}\left(G_{\mu}\right) \cong$ ${ }^{2} A_{3}(3) \cong U_{4}(3)$ satisfies $L_{\lambda} \cong B_{3}(3)$. However, $L$ has an automorphism $\eta$ of order $2, \eta \equiv \lambda(\bmod \operatorname{Inn}(L))$, such that $L_{\eta} \cong{ }^{2} D_{2}(3) \cong A_{6}$. There is a subgroup $M<L$ containing $L_{\eta}, M \cong P S L(3,4)$. The existence of this $M$ is not easily predicted by a study of the Lie structure. Indeed, its existence led J. E. McLaughlin to construct a sporadic simple group [21]. Looking at this example in more detail, we see that ${ }^{2} A_{3}(3)={ }^{2} D_{3}(3)$, so that $L$ may be regarded as $K / Z(K)$, where $K=\Omega^{-}(6,3)$, the commutator subgroup of the orthogonal group $O^{-}(6,3)$. In terms of matrices, let $B$ be any symmetric $4 \times 4$ nonsingular matrix of determinant -1 with entries from $\boldsymbol{F}_{3}$ and let be the result of applying the field automorphism $x \mapsto x^{3}$ to a $4 \times 4$ 
matrix with entries from $\boldsymbol{F}_{9}$. Then $S U(4,3)$ may be identified with $\left\{\left.A\right|^{t} \bar{A} B A=B, \operatorname{det} A=1\right\}$ and it has a "natural" field automorphism $\varphi: A \rightarrow \bar{A}$. However, $\varphi$ is not the "standard field automorphism" of $S U(4,3)$, as we have defined the term above. In fact, the fixed points of $\varphi$ is the special orthogonal group associated with $B$. See Artin [1], p. 210.

A variation of our situation is the following: $M$ is a group lying between $O^{p^{\prime}}\left(G_{\lambda, \mu}\right)^{\prime}$ and $O^{p^{\prime}}\left(G_{\mu}\right)$. The problem (still not fully solved) is to show that $O^{p^{\prime}}\left(G_{\lambda, \mu}\right)^{\prime} \triangleleft M$ or identify $M$.

Of course, any "interesting" exceptions will be ones not already described by our main theorem. That is, we will be dealing with a Chevalley or twisted group $O^{p^{\prime}}\left(G_{\lambda, \mu}\right)$ which is not perfect (i.e., is not equal to its commutator subgroup). The possibilities for $O^{p^{\prime}}\left(G_{\lambda, \mu}\right)$ are then the solvable groups $A_{1}(2)^{\prime}, A_{1}(3)^{\prime},{ }^{2} A_{2}(2)$, and ${ }^{2} C_{2}(2)$, plus the nonsolvable groups $B_{2}(2) \cong \Sigma_{6}, G_{2}(2) \cong \operatorname{Aut}\left(U_{3}(3)\right),{ }^{2} G_{2}(3) \cong \operatorname{Aut}\left(L_{2}(8)\right)$ and ${ }^{2} F_{4}(2)^{\prime}$. The only exception known to the authors, for $O^{p^{\prime}}\left(G_{\lambda, \mu}\right)$ nonsolvable, is

$$
G_{2}(2)^{\prime}<M<G_{2}(4), \quad M \cong J_{2} \text {, Janko simple group }
$$

group of order 604,800; there are two conjugacy classes of such $M$, see Wales [27].

We mention that [27] does not determine all maximal subgroups of $G_{2}(4)$ containing $G_{2}(2)^{\prime}$.

Another example we mention is the containment

$$
{ }^{2} F_{4}(2)^{\prime}<M<{ }^{2} E_{6}(2),
$$

where $M \cong M(22)$, the Fischer group of order $2^{17} 3^{9} 5^{2} \cdot 7 \cdot 11 \cdot 13$ [9], [10]. This does not quite fit in the above situation, because ${ }^{2} F_{4}(2)$ cannot be realized as $G_{\lambda, \mu}$, where $G=E_{6}(k)$, char $k=2$. However, the questions to be asked here are obvious: find finite groups $M$ (if any) for which ${ }^{2} F_{4}(2)^{\prime}<M<X$, where $X \cong{ }^{2} F_{4}(q), F_{4}(q),{ }^{2} E_{6}(q)$ and $E_{6}(q)$, for $q$ even, and where ${ }^{2} F_{4}(2)^{\prime}<{ }^{2} F_{4}(2)$ is embedded in the natural fashion in $X$. We point out that in the above case where $M \cong M(22)$, it is not known for certain that the ${ }^{2} F_{4}(2)^{\prime}$ subgroup of $M$ is conjugate to the one embedded in the "natural" way in ${ }^{2} E_{6}(2)$.

(3.3) Proof of Theorem 2. We proceed by a series of lemmas. Some important intermediate results are given in Propositions 3.1 and 3.2 .

LemMa 3.1. Suppose $G$ has a root system $\Sigma$ having one root length. Let $\mu={ }^{s} \sigma_{q}, s \in\{2,3\}$, and let $\lambda=\sigma_{q}$. Suppose $M$ is a subgroup of $G$ such that $G_{\lambda, \mu}^{s} \subseteq M \subset G_{\mu}^{s}$. Then one of the following holds: 
(a) $p \nmid\left|M: G_{\lambda, \mu}^{s}\right|$

(b) $p=2, \Sigma=A_{2 n}$, and either $O^{2^{\prime}}(M) \cong{ }^{2} A_{2 n-1}(q)$, or $G_{\mu}={ }^{2} A_{2}(2)$.

Proof. Let $\bar{\Sigma}$ be the twisted "root system" of $G_{\mu}$ and $\bar{W}$ the corresponding Weyl group. Thus $N_{\mu} / H_{\mu} \cong N_{\lambda, \mu} / H_{\lambda, \mu} \cong \bar{W}$. Also, $U_{\mu}=$ $\prod_{\rho \in \bar{\Sigma}} x_{\rho}$. If $\Sigma \neq A_{2 n}$, then $\bar{\Sigma}$ is a bona fide root system, and $X_{\rho}$ is parametrized by $G F(q)$ for long $p$, by $G F\left(q^{s}\right)$ for short $\rho$. If $\Sigma=A_{2 n}$, then $s=2$, and $\bar{\Sigma}=\left\{ \pm\left(a_{i}, 2 \alpha_{i}\right)\right.$, $\left.\pm a_{i} \pm a_{j} \mid 1 \leqq i<j \leqq n\right\}$ is of type " $B C_{n}$ ", with $X_{ \pm a_{i} \pm a_{j}}$ parametrized by $G F\left(q^{2}\right)$ and $\dot{X}_{ \pm\left(a_{i}, 2 a_{i}\right)}$ of type ${ }^{2} A_{2}$. The parametrizations by $G F\left(q^{s}\right)$ are not quite canonical: if $\tau$ is the Frobenius automorphism of $G F\left(q^{s}\right) / G F(q)$ there are $s$ canonical parametrizations of $X_{\rho}$, in which the same element is represented as $x_{\rho}(t)$, or $x_{\rho}\left(t^{\tau}\right)$ (or $X_{\rho}\left(t^{\tau^{2}}\right)$ if $s=3$ ). We shall ignore this ambiguity since it does not affect the validity of our arguments. Note that if $X_{\rho}$ is pärametrized by $G F(q)$, then $\left(X_{\rho}\right)_{\mu}=X_{\rho}$; while if by $G F\left(q^{s}\right)$, then $\left(X_{\rho}\right)_{\mu}=\left\{x_{\rho}(t) \mid t \in G F(q)\right\}$.

We show first that $N_{G_{u}}\left(U_{\lambda, \mu}\right) \subseteq B_{\mu}$. Let $g \in N_{G_{\mu}}\left(U_{\lambda, \mu}\right)$, and write $g=b n_{w} u$ in canonical form $(w \in \bar{W})$. For every fundamental $\rho \in \bar{\Sigma}$, let $U^{o}=\prod_{\substack{o \neq \rho \\ \sigma>0}} X_{o}$, so that $U_{\rho} \triangleleft U, U=U^{o} X_{\rho}$, and $X_{\rho} \cap U_{\rho}=1$. (In case $\Sigma=B C_{n}^{>0}$ we take $\left\{\left(a_{1}, 2 a_{1}\right), a_{2}-a_{1}, \cdots, a_{n}-a_{n-1}\right\}$ as the fundamental system.) Now $U_{\lambda, \mu} \cap X_{\rho} \neq 1$ for each such $\rho$, so $\left(U_{\lambda, \mu}\right)^{b}$ contains an element of the form $x_{\rho} u_{\rho}$ with $1 \neq x_{\rho} \in X_{\rho}, u_{\rho} \in U^{\rho}$. Since $\left(x_{\rho} u_{\rho}\right)^{n} w \in\left(U_{\lambda, \mu}\right)^{u-1} \leqq U, w(\rho) \in \overline{\bar{\Sigma}}^{+}$. Hence $w=1$, so $g \in \ddot{B}_{\mu}$.

Now suppose (a) fails. Let $U^{*}=N_{M \cap U_{\mu}}\left(U_{\lambda, \mu}\right)$. Since $U_{\lambda, \mu}$ is not one of $N_{\dot{\mu}}\left(U_{\lambda, \mu}\right)$ which equals $N_{M \cap B_{\lambda}}\left(U_{\lambda, \mu}\right)$ by the above. Since $U_{\mu}$ is the Sylow $p$-subgroup of $B_{\mu}, U^{*} U_{\lambda, \mu}$.

Suppose $\Sigma \neq A_{2 n}$. Put a partial order $\leqq$ on $\bar{\Sigma}$ refining the order given by heights. Write each $u \in U_{\mu}$ as $u=\Pi_{\bar{\Sigma}}+x_{\rho}\left(t_{\rho}\right)$ in order, and set $\operatorname{supp}(u)=\left\{\rho \mid t_{\rho} \neq 0\right\}$. Among all elements of $U^{*}-U_{\lambda, \mu}$, choose $x$ to have the greatest support, in the lexicographic ordering. Write $x=x_{\rho_{0}}\left(t_{\rho_{0}}\right) \prod_{\rho>\rho_{p}} x_{\rho}\left(t_{\rho}\right)$ with $t_{\rho_{0}} \neq 0$. Then in fact $x_{\rho_{0}}\left(t_{\rho_{0}}\right) \notin U_{\lambda, \mu}$, otherwise $x^{\prime}=x_{\rho_{0}}\left(-t_{\rho_{0}}\right) x \in U^{*}-U_{\lambda, \mu}$, and $\operatorname{supp}\left(x^{\prime}\right)>\operatorname{supp}(x)$, contrary to choice of $x$. In particular, $t_{\rho_{0}} \notin G F(q)$, so $\rho_{0}$ is short. Suppose there is $\sigma \in \bar{\Sigma}^{+}$such that $\rho_{0}$ and $\sigma$ are fundamentally independent. Let $x^{*}=\left[x_{o}(1), x\right]=x_{\rho_{0}+\sigma}\left( \pm t_{\rho_{0}}\right) \cdots$, (for a complete description of the commutator formula in Steinberg variations, see [15]). Then $x_{\sigma}(1) \epsilon$ $U_{\lambda, \mu}$ and $x \in U^{*}$ imply $x^{*} \in U_{\lambda, \mu}$, so $t_{\rho_{0}} \in G F(q)$, contradiction. Hence no such $\sigma$ is available. Suppose $\bar{\Sigma}=G_{2}$, with fundamental system $\{\alpha, \beta\}, \beta$ short, and $\rho_{0}=\alpha+\beta$. Then $x_{\beta}(1), x_{\alpha+2 \beta}(1) \in U_{\lambda, \mu}$, so $U_{\lambda, \mu}$ contains both $\left[x_{\alpha}(1), x\right]=x_{\alpha+2 \beta}\left( \pm\left(t_{\rho_{0}}^{\tau}+t_{\rho_{0}}^{\tau^{2}}\right)\right)$ and

$$
\left[x_{\alpha+2 \beta}(1), x\right]=x_{2 \alpha+3 \beta}\left( \pm\left(t_{\rho_{0}}+t_{\rho_{0}}^{\tau}+t_{\rho_{0}}^{\tau^{2}}\right)\right)
$$

Hence $G F(q)$ contains both coefficients, so contains $t_{\rho_{0}}$, contradiction. 
We conclude from $\left({ }^{*}\right)$ (see Lemma 2.1) that $\rho_{0}=\theta_{s}$. In the factorization of $x$, all terms $x_{\rho}\left(t_{\rho}\right)$ after the first are for long $\rho$, hence lie in $U_{\lambda, \mu}$. Hence $x_{\rho_{0}}\left(t_{\rho_{0}}\right)^{-1} x \in U_{\lambda, \mu}$, so $x_{\rho_{0}}\left(t_{\rho_{0}}\right) \in U^{*}$. Hence $X_{\rho_{0}} \cap M \supset\left(X_{\rho_{0}}\right)_{\lambda}$. Now $\left\langle X_{\rho_{0}}, X_{-\rho_{0}}\right\rangle \cong A_{1}\left(q^{s}\right)$, and $\lambda$ induces a field automorphism $\sigma_{q}$ on this group, so by Theorem 1 (more precisely Lemma 2.5, which holds even for $q=2),\left\langle X_{\rho_{0}}, X_{-\rho_{0}}\right\rangle \leqq M$, as $s$ is prime. Conjugating by $N_{\lambda, \mu}$, we get $X_{\rho} \subseteq M$ for all short $\rho$; since $X_{\rho}=\left(X_{\rho}\right)_{\lambda} \subseteq M$ for long $\rho, M=G_{\mu}^{s}$, contrary to hypothesis. Therefore, $\Sigma=A_{2 n}$.

If $n=1$, then (b) is immediate from work of Mitchell [22] and Hartley [16]. Suppose then $n>1$. For a root $\rho= \pm a_{i} \pm a_{j}, X_{\rho}=$ $\left\{x_{\rho}(t) \mid t \in G F\left(q^{2}\right)\right\}$ and $\left(X_{\rho}\right)_{\lambda}=\left\{x_{\rho}(t) \mid t \in G F(q)\right\}$. For each $i=1, \cdots, n$, there is a root subgroup $X_{i}=\left\{x_{i}(t, u) \mid t^{1+q}+u+u^{q}=0, t, u \in G F\left(q^{2}\right)\right\}$ corresponding to the "root" $\left(a_{i}, 2 a_{i}\right)$. The opposite root subgroup is denoted by $X_{-i}$. We separate $X_{i}$ into parts $X_{a_{i}}$ and $X_{2 a_{i}}$ as follows: let $X_{2 a_{i}}=Z\left(X_{i}\right)=\left\{x_{i}(0, u) \mid u \in G F\left(q^{2}\right), u+u^{q}=0\right\}$, and write $x_{2 a_{i}}(u)$ for $x_{i}(0, u)$. Let $X_{a_{i}}$ be a transversal to $X_{2 a_{i}}$ in $X_{i}$. If $q$ is odd, we may choose $X_{a_{i}}$ to be $\mu$-invariant, so that if a coset $C$ of $X_{2 a_{i}}$ in $X_{i}$ is fixed by $\lambda$, then the representative of $C$ in $X_{a_{i}}$ is fixed by $\lambda$. The element of $X_{a_{i}}$ representing the coset $x_{i}(t, u) X_{2 a_{i}}$ will be written $x_{i}(t)\left(t \in G F\left(q^{2}\right)\right)$. Thus $X_{i}$ is parametrized by $G F\left(q^{2}\right)$. We choose $x_{i}(0)=1$, without loss.

Let $\widetilde{\Sigma}=\left\{ \pm a_{i}, \pm 2 a_{i}, \pm a_{i} \pm a_{j} \mid 1 \leqq i<\leqq n\right\}$. Define a height function on $\tilde{\Sigma}$ by setting $h t\left(a_{i}\right)=i$ and extending linearly. Then for $\rho, \sigma \in \widetilde{\Sigma}^{+},\left[X_{\rho}, X_{\sigma}\right] \leqq\left\langle X_{\alpha} \mid \alpha \in \widetilde{\Sigma}, h t(\alpha) \geqq h t(\rho)+h t(\sigma)\right\rangle$. Let $\leqq$ be a partial order on $\widetilde{\Sigma}$ refining the height order. Since $X_{ \pm a_{i} \pm a_{j}}, X_{2 a_{i}}$, and $X_{i}=X_{a_{i}} X_{2 a_{i}}$ are subgroups of $G_{\mu}$, and since $a_{i}<2 a_{i}$, every $u \in U_{\mu}$ is uniquely expressable as $\Pi x_{\rho}\left(t_{\rho}\right)$, the product over $\rho \in \widetilde{\Sigma}^{+}$in increasing order, with $t_{\rho}$ in the appropriate field. Set $\operatorname{supp}(u)=$ $\left\{\rho \mid t_{\rho} \neq 0\right\}$. Again, among all $x \in U^{*}-U_{\lambda, \mu}$ choose $x$ maximal in the lexicographic ordering. Say $x=x_{\rho_{0}}\left(t_{\rho_{0}}\right) \prod_{\rho>\rho_{0}} x_{\rho}\left(t_{\rho}\right)$, with $t_{\rho_{0}} \neq 0$. Then as before, $x_{\rho_{0}}\left(t_{\rho_{0}}\right) \notin U_{\lambda, \mu}$.

Suppose $q$ is odd. Then $\left(X_{i}\right)_{\lambda}=\left(X_{a_{i}}\right)_{\lambda}=\left\{x_{a_{i}}(t) \mid t \in G F(q)\right\}$ for each $i$. So $x_{a_{i}}(1) \in U_{\lambda, \mu}$ for all $i$. Suppose $\rho_{0}=\alpha_{j}-a_{i}$ for some $j>i$. Then $\left[x, x_{a_{i}}(1)\right]=x_{a_{j}}\left( \pm t_{\rho_{0}}\right) \cdots$ lies in $U_{\lambda, \mu}$ so $t_{\rho_{0}} \in G F(q)$, whence $x_{\rho_{0}}\left(t_{\rho_{0}}\right) \in U_{\lambda, \mu}$, contradiction. If $\rho_{0}=a_{i}$, then for $j=1$ or $2, U_{\lambda, \mu}$ contains $\left[x, x_{a_{j}}(1)\right]=x_{a_{i}+a_{j}}\left( \pm t_{\rho_{0}}\right) \cdots$, so $t_{\rho_{0}} \in G F(q)$ and $x_{\rho_{0}}\left(t_{\rho_{0}}\right) \in U_{\lambda, \mu}$, contradiction. If $\rho_{0}=a_{i}+a_{j}, j>i$, then $U_{\lambda, \mu}$ contains $\left[x, x_{a_{j}-a_{i}}(1)\right]=$ $x_{2 a_{j}}\left( \pm\left(t_{\rho_{0}}-t_{\rho_{0}}^{q}\right)\right) \cdots$. Since $\left(X_{2 a_{j}}\right)_{\mu}=1, t_{\rho_{0}}-t_{\rho_{0}}^{q}=0$, so $t_{\rho_{0}} \in G F(q)$, again giving a contradiction. Suppose $\rho_{0}=2 a_{i}, 1 \leqq i<l$. Write $x=x_{2 a_{i}}\left(t_{\rho_{0}}\right) \cdots x_{a_{i}+a_{i+1}}(t) \cdots$. Then

$$
\left[x, x_{a_{i+1}-a_{i}}(1)\right]=x_{a_{i}+a_{i+1}}\left( \pm t_{\rho_{0}}\right) \cdots x_{2 a_{i+1}}\left( \pm\left(t-t^{q}\right) \pm t_{\rho_{0}}\right) \cdots
$$

lies in $U_{\lambda, \mu}$, so $t_{\rho_{0}} \in G F(q)$ and $t-t^{q} \pm t_{\rho_{0}}=0$. Hence $t-t^{q} \in G F(q)$. Since $q$ is odd, this implies $t-t^{q}=0$. Hence $t_{\rho_{0}}=0$, contradiction. 
We conclude that $\rho_{0}=2 a_{n}$. Hence $M \cap X_{n} \supset\left(X_{n}\right)_{\lambda}(=1)$. Applying the case $n=1$ to $\left\langle X_{n}, X_{-n}\right\rangle$, we get $\left\langle X_{n}, X_{-n}\right\rangle \subseteq M$. Conjugating by $N_{\lambda, \mu}$, we get $X_{i} \subseteq M$ for all $i$. Hence $M$ contains $\left[x_{a_{1}}(t), x_{a_{2}}\left(t^{\prime}\right)\right]=$ $x_{a_{1}+a_{2}}\left( \pm t t^{\prime}\right)$ for all $t, t^{\prime} \in G F\left(q^{2}\right)$, so $X_{a_{1}+a_{2}} \subseteq M$. This easily yields $G_{\mu}^{s}=M$, contradiction. Therefore, $q$ is even, i.e., $p=2$.

In this case, we have $\left(X_{i}\right)_{\lambda}=X_{2 a_{i}}$, and $X_{a_{i}}$ is not $\lambda$-invariant. Let $x, \rho_{0}$, and $t_{\rho_{0}}$ be as before. If $\rho_{0}=a_{j}-a_{i}$ for some $j>i$, then $U_{\lambda, \mu}$ contains $\left[x, x_{2 a_{i}}(1)\right]=x_{a_{j}+a_{i}}\left(t_{\rho_{0}}\right) \cdots$, so $t_{\rho_{0}} \in G F(q)$, contradiction. If $\rho_{0}=2 a_{i}$, then $x_{\rho_{0}}\left(t_{\rho_{0}}\right) \in X_{2 a_{i}} \subseteq U_{\lambda, \mu}$, contradiction. If $\rho_{0}=a_{i}+a_{j} \neq a_{n-1}+a_{n}$, then there exists $\sigma=a_{j^{\prime}}-a_{i^{\prime}}, j^{\prime}>i^{\prime}$, such that $\rho_{0}+\sigma$ is of the form $a_{k}+a_{l}$, and so $U_{\lambda, \mu}$ contains $\left[x, x_{o}(1)\right]=$ $x_{\rho_{0}}+\sigma\left(t_{\rho_{0}}\right) \cdots$, contradiction. If $\rho_{0}=a_{i}, 1 \leqq i<n$, then $U_{\lambda, \mu}$ contains $\left[x, x_{a_{i+1}-a_{i}}(1)\right]=x_{a_{i+1}}\left(t_{\rho_{0}}\right) \cdots$, contradiction. Suppose $\rho_{0}=a_{n}$, and write $x=x_{a_{n}}\left(t_{\rho_{0}}\right) \cdots x_{2 a_{n}}\left(t^{\prime}\right), x_{a_{n}}\left(t_{\rho_{0}}\right)=x_{n}\left(t_{\rho_{0}}, u\right)$. Then $u+u^{q}=t_{\rho_{0}}^{1+q} \neq 0$, so $u \in G F\left(q^{2}\right)-G F(q)$. Let $n_{0}=n_{a_{n-a_{n-1}}}(1)$, and set $x^{\prime}=x^{n_{0}}=x_{a_{n-1}}\left(t_{\rho_{0}}\right) \cdots$ $x_{2 a_{n-1}}\left(t^{\prime}\right)$ (with other nontrivial terms coming only from roots of the form $a_{i}+a_{j}$ or $\left.2 a_{i}\right)$. Let $x^{(2)}=\left[x^{\prime}, x_{a_{n-a_{n-1}}}(1)\right]$. Then $x^{(2)} \in M$, and $x^{(2)}=x_{a_{n}}\left(t_{\rho_{0}}\right) \cdots x_{a_{n}+a_{n-1}}\left(t^{\prime q}+u^{q}\right) x_{2 a_{n}}()$, with inside nontrivial terms coming only from roots of the form $a_{n}+a_{j}$ Let $u^{\prime}=t^{q}+u^{q}$. Since $t^{\prime} \in G F(q)$ and $u \notin G F(q), u^{\prime} \notin G F(q)$. Now set $n_{1}=n_{a_{n-1}}(1)$, and $x^{(3)}=$ $\left[x^{\prime},\left(x^{(2)}\right)^{n_{1}}\right]$. Then $x^{(3)} \in M$, and $x^{(3)}=x_{a_{n}}\left(t_{\rho_{0}} u^{\prime}\right) \cdots$. Since $u^{\prime} \notin G F(q)$, we may assume that $t_{\rho_{0}} \notin G F(q)$, by replacing $x$ by $x^{(3)}$ at the outset if necessary. But then $\left[x, x^{n_{0}}\right]=x_{a_{n}+a_{n-1}}\left(t_{\rho_{0}}^{2}\right)$ and $t_{\rho_{0}}^{2} \in G F(q)$, so the maximality of $x$ is violated. Thus $\rho_{0} \neq a_{n}$, so $\rho_{0}=a_{n}+a_{n-1}$. Hence $x_{\rho_{0}}\left(t_{\rho_{0}}\right)=x \cdot x_{2 a_{n}}() \in U^{*}-U_{\lambda, \mu \cdot}$ Applying Theorem 1 (Lemma 2.5) to $\left\langle X_{a_{n}+a_{n-1}}, X_{-a_{n}-a_{n-1}}\right\rangle$, we see that $X_{a_{n}+a_{n-1}} \subseteq M$. Thus $X_{\rho} \subseteq M$ if $\rho= \pm a_{i} \pm a_{j}$. Let $\widetilde{G}=\left\langle X_{\rho}\right| \rho= \pm a_{i} \pm a_{j}$ or $\left.2 a_{i}\right\rangle$, so that $\widetilde{G} \cong M$, and $\widetilde{G}$ is (canonically generated) ${ }^{2} A_{2 n-1}(q)$. It is easily verified that $N_{G_{\mu}}(\widetilde{G})$ is the unique maximal subgroup of $G_{\mu}$ containing $\widetilde{G}$. One considers the permutation group induced by $S U(2 n+1, q)$ on anisotropic vectors of a given length in the natural $2 n+1$-dimensional module over $G F\left(q^{2}\right)$, and shows that the only sets of imprimitivity have the property that every block is a subset of one-dimensional subspace. Hence $\widetilde{G} \subseteq M \cong N_{G_{\mu}}(\widetilde{G})$. Since $N_{G_{\mu}}(\widetilde{G}) / \widetilde{G} \cong Z_{q+1}$ is of odd order, $\widetilde{G}=O^{2}(M)$, completing the proof.

We are now entitled to work under the following conditions:

(A) $r>1$ is an integer

(B) $\lambda, \mu$ are commuting endomorphisms of $G$ with $G_{\lambda}$ and $G_{\mu}$ finite and $\lambda$ induces an automorphism of order $r$ on $G_{\mu}$

(C) Either (i) $\lambda^{r}=\mu$ and $\lambda=\sigma_{q}$ or $\lambda={ }^{8} \sigma_{q}$ where $r \nmid s$ and the Dynkin diagram for $G$ has period $s \in\{2,3\}$; or (ii) $\lambda=\sigma_{q}$ and $\mu=$ ${ }^{s} \sigma_{q^{r / s}}$, where $r \mid s$ and the Dynkin diagram for $G$ has period $s \in\{2,3\}$. (D) $O^{p^{\prime}}\left(G_{\lambda, \mu}\right) \leqq M \leqq G_{\mu}$ 
(E) $|M|_{p}=\left|G_{\lambda, \mu}\right|_{p}$ i.e., $U_{\lambda, \mu} \in \operatorname{Syl}_{p}(M)$.

First a few observations. Namely, $G_{\lambda, \mu}$ and $G_{\mu}$ have the same rank and consequently, if $P$ is a $\langle\lambda, \mu\rangle$-invariant parabolic subgroup of $G, \lambda$ leaves invariant every component of $P_{\mu} / O_{p}\left(P_{\mu}\right)$ (see 2.4 for a discussion of components). We do not assume $r$ is a prime. Here, the critical assumption is that $M_{\lambda, \mu}=M \cap G_{\lambda, \mu}$ contains a Sylow $p$ group of $M$. Also, even though Theorem 1 deals with the above case (C. i), none of the following arguments, except Lemma 3.9 and Proposition 3.2 are simplified by quoting Theorem 1 .

LEMma 3.2. Let $P_{\mu}$ be a proper parabolic subgroup of $G_{\mu}$ containing $B_{\mu} . \quad$ Write $P_{\mu}=O_{p}\left(P_{\mu}\right) \cdot L_{\mu}$, where $L_{\mu}$ is generated by $H_{\mu}$ and standard root groups from $G_{\mu}$. Let $\Sigma_{\mu}$ be a root system for $G_{\mu}$. Let $\Sigma_{0}=\left\{r \in \Sigma_{\mu} \mid X_{r} \leqq O_{p}\left(P_{\mu}\right)\right\}$, where $X_{r}$ denotes a root group for $G_{\mu}$ (rather than for $G$ ). Set $P_{\mu}^{-}=\left\langle X_{r}, H_{\mu} \mid X_{-r} \leqq P_{\mu}\right\rangle$. Then $G_{\mu}=\left\langle O_{p}\left(P_{\mu}\right)\right.$, $\left.O_{p}\left(P_{\mu}^{-}\right)\right\rangle$.

Proof. Let $S=\left\langle O_{p}\left(P_{\mu}\right), O_{p}\left(P_{\mu}^{-}\right)\right\rangle$. Then $L_{\mu}$ normalizes $S$, whence $S L_{\mu}$ is a group containing $B_{\mu}$, i.e., $S L_{\mu}$ is a standard parabolic subgroup. If $S L_{\mu}$ were proper, then $O_{p}\left(S L_{\mu}\right)$ would meet $X_{\alpha}$ nontrivially, for some $\alpha \in \Sigma_{0}$. But $X_{-\alpha} \leqq S$ implies that $O_{p}\left(\left\langle X_{\alpha}, X_{-\alpha}\right\rangle\right)=1$, contradiction. Thus $S L_{\mu}=G$. Since $S \triangleleft S L_{\mu}, S=G_{\mu}$, as required.

LEMMA 3.3. Let $P$ be proper parabolic subgroup of $G$ containg B. Then $C_{\theta_{\mu}}\left(O_{p}\left(P_{\mu}\right)\right) \leqq O_{p}\left(P_{\mu}\right)$, i.e., $O_{p^{\prime}}\left(P_{\mu}\right)=1$ and $P_{\mu}$ is p-constrained.

Proof. If necessary, we shall replace $\mu$ by $\nu=\mu^{j}$, where $j>1$ is an integer such that (i) if $\mu$ involves a graph automorphism of period $s>1,(j, s)=1$ (ii) in $G_{\nu}$, two opposite root groups generate a quasisimple group, i.e., we are avoiding small fields. Note that $G_{2}$ and $G_{\mu}$ have the same Weyl group and $G_{\mu} \leqq G_{\nu}$. We claim that this change affects neither hypothesis nor conclusion. Namely, set $C_{\tau}=$ $C_{G_{\tau}}\left(O_{p}\left(P_{\tau}\right)\right) \triangleleft P_{\tau}$ for $\tau \in\{\mu, \nu\}$. By the fact that if $X_{\mu}$ is a root group for $G_{\nu}$ and $X_{\mu}=\left(X_{\nu}\right)_{\mu}, C_{G_{\nu}}\left(X_{\mu}\right)=C_{G_{\nu}}\left(X_{\nu}\right)$ (a straightforward exercise) and the fact that $O_{p}\left(P_{\tau}\right)$ is a product of root groups in $G_{\tau}, \tau \in\{\mu, \nu\}$, we get $C_{\mu}=C_{\nu} \cap G_{\mu}$. Thus, it suffices to prove $C_{\nu} \leqq O_{p}\left(P_{\nu}\right)$, because then $C_{\mu}$ is a normal $p$-group in $P_{\mu}$, whence $C_{\mu} \leqq O_{p}\left(P_{\mu}\right)$. So, we make the replacement.

Let $r$ be a root in the root system $\Sigma_{\mu}$ and $X_{r}$ the corresponding root group in $G_{\mu}$. An element of $H_{\mu}$ centralizes $X_{r}$ if and only if it centralizes $X_{-r}$. Therefore, by Lemma 3.2, $C \cap H_{\mu} \leqq Z(G)=1$. Letting - denote the quotient $P_{\mu} \rightarrow \bar{P}_{\mu}=P_{\mu} / O_{p}\left(P_{\mu}\right)$, we claim that $\bar{C} \cap \bar{H}_{\mu}=1$. If not, let $H_{0} \leqq H_{\mu}$ satisfy $\bar{H}_{0}=\bar{C} \cap \bar{H}_{\mu}$. Now, $C$ is a normal subgroup of $p$-power index in $C \cdot O_{p}\left(P_{\mu}\right)$, whence $H_{0} \leqq C$, and 
so $C \cap H_{\mu} \neq 1$, absurd. Thus $\bar{C} \cap \bar{H}_{\mu}=1$. It follows that $\bar{C} \cap O^{p^{\prime}}\left(\bar{P}_{\mu}\right)=$ 1 , because our replacement of $\mu$ guarantees that any normal subgroup of $O^{p^{\prime}}\left(\bar{P}_{\mu}\right)$ lies in $\bar{H}_{\mu}$. Therefore, $\left[\bar{C}, \bar{U}_{\mu}\right]=1$. This means $C \leqq B_{\mu}$. Since $B_{\mu}$ has a normal Sylow $p$-subgroup and $O_{p}(\bar{C})=1$, it follows that $\bar{C}$ is a normal $p^{\prime}$-subgroup of $\bar{B}_{\mu}$, whence $1 \neq \bar{C} \leqq \bar{H}_{\mu}$, in conflict with above statements. The lemma follows.

Lemma 3.4. (i) For any $\mu, U$ is the unique conjugate of $U$ which contains $U_{\mu}$. (ii) Also $U$ is the unique conjugate of $U$ which contains $U_{\lambda, \mu}$, unless $q$ is even, $\lambda=\sigma_{q}, \mu={ }^{2} \sigma_{q r / s}$ and $G$ has type $A_{2 n}$, in which case $\left\{g \in G \mid U_{\lambda, \mu} \leqq U^{g}\right\}=B \cup B n_{w_{r}} B \cup n_{w_{s}} B$, where $\left\{1, w_{r}, w_{s}\right\}=\left\{w \in\left\langle w_{r}, w_{s}\right\rangle \mid X_{r+s}^{w} \leqq\left\langle X_{r}, X_{s}\right\rangle\right\}$ where $r, s$ are the $n$th and $(n+1)$ st roots in the Dynkin diagram for $G$. (iii) However, in all cases, $U_{\mu}$ is the unique $G_{\mu}$-conjugate of $U_{\mu}$ containing $U_{\lambda, \mu}$.

Let $P(\lambda, \mu)$ be a parabolic subgroup for $G_{\lambda, \mu}$. (iv) Then there is a unique parabolic subgroup $P(\mu)$ of $G_{\mu}$ which contains $P(\lambda, \mu)$, and satisfies $P(\mu)_{\lambda}=P(\lambda, \mu)$. (v) Also there is a unique $\langle\lambda, \mu\rangle$-invariant parabolic subgroup $P$ of $G$ for which $P_{\lambda, \mu}=P(\lambda, \mu)$ and $P=\langle P(\lambda, \mu), B\rangle$, unless we have the above exceptional $q, G, \lambda, \mu$ (see (ii)) and the $P(\lambda, \mu)$ is the one containing $B_{\lambda, \mu}$ which is associated with the subset of the Dynkin diagram for $G$ consisting of all short roots. In the exceptional case, there is a $\langle\lambda, \mu\rangle$-invariant parabolic subgroup of $G$ for which $P_{\lambda, \mu}=P(\lambda, \mu), e . g ., P=\left\langle P(\lambda, \mu), B^{g}\right\rangle$, where $g \in G_{\lambda, \mu}$ satisfies $B_{\lambda, \mu}^{g} \leqq P$.

Proof. (ii) Let $U_{\mu}<V=U^{g}, g \in G$. Let $\Sigma$ be a root system for $G$. Write $g=b n_{w} u$, where $b \in B, n_{w} \in N_{G}(H)$ represents the element $w$ of the Weyl group, and $u \in U(w)=\left\langle X_{\alpha} \mid \alpha \in \Sigma^{+}, \alpha^{w^{-1}} \in \Sigma^{-}\right\rangle$. Let $U^{(w)}=\left\langle X_{\alpha} \mid \alpha \in \Sigma^{+}, \alpha^{w-1} \Sigma^{+}\right\rangle$. Then $U^{g}=U^{n_{w} u}$ and so $U_{\mu} \leqq U^{(w) u}$. Suppose $g \notin B$. Then there is such a $g$ for which $w$ is a fundamental reflection, $w=w_{\alpha}$ (see the appendix of Steinberg's notes [24]) so that $U^{(w)} \triangleleft U$. Thus to get a contradiction, it suffices to show $U_{\lambda, \mu} \not \equiv U^{(w)}$.

Write $X_{r}=U_{(w)}$. If $\langle\lambda, \mu\rangle$ leave $X_{r}$ invariant, we are done, as $\left(X_{r}\right)_{\lambda} \neq 1$. Therefore $\mu={ }^{s} \sigma_{q^{\prime}}$ where $q^{\prime}$ is some power of $p$ and $s=2$ or 3. But now, we see that $R=\left\langle X_{r}^{\mu i} \mid 0 \leqq i \leqq-1\right\rangle$ satisfies $R_{\lambda, \mu}$ $U^{(w)}$ by checking the possibilities, unless $G=A_{2 n}(k), n \geqq 1, \mu={ }^{2} \sigma_{q} r / 2$ and $\lambda=\sigma_{q}$ and $r$ is the $n$th or $(n+1)$ st node in the Dynkin diagram for $A_{2 n}$. The verification of the rest of (i) and (ii) is an exercise.

The proof of (iii) is obtained by a similar argument, and (iv) and (v) are straightforward.

LEMMA 3.5. There does not exist a proper parabolic subgroup of $G_{\mu}$ containing $G_{\lambda}$. 
Proof. Assume false, and take a parabolic subgroup $R, G_{\lambda} \leqq$ $R<G_{\mu}$. Embed $U_{\lambda}$ in a Sylow $p$-subgroup of $R$. By Lemma 3.4, $U_{\lambda}<U_{\mu}<R$. Since $R$ is a proper parabolic subgroup, it is $p$-constrained (by Lemma 3.3) whence $Z(U) \leqq O_{p}(R)$. Thus $1 \neq Z(U)_{\lambda} \leqq$ $O_{p}(R) \cap G_{\lambda} \triangleleft G_{\lambda}$, whereas $O_{p}\left(G_{\lambda}\right)=1$, contradiction.

Lemma 3.6. Let $P$ be a parabolic subgroup of $G$ which is $\langle\lambda, \mu\rangle-$ invariant. Then $O_{p}\left(P_{\lambda}\right)=O_{p}(P)_{\lambda}, O_{p}\left(P_{\mu}\right)=O_{p}(P)_{\mu}, O_{p}\left(P_{\lambda, \mu}\right)=O_{p}(P)_{\lambda, \mu}$.

Proof. Clearly $O_{p}(P)_{\lambda} \leqq O_{p}\left(P_{\lambda}\right)$. Suppose the containment is proper. Let ${ }^{-}$denote the quotient map $P \rightarrow P / O_{p}(P)$. Then $\overline{O_{p}\left(P_{\lambda}\right)} \neq 1$ is a normal $p$-subgroup of $\bar{P}$. However, $\langle\lambda, \mu\rangle$ leaves invariant a complement $L$ to $O_{p}(P)$ in $P$. The structure of $L$ implies that $O_{p}\left(L_{\lambda}\right)=1$, contradiction. So $O_{p}\left(P_{\lambda}\right)=O_{p}(P)_{\lambda}$. The other assertions are proven similarly.

LEMMA 3.7. Let $V \leqq H_{\mu}$ be a group of order prime to $p$ for which $\left[U_{\lambda, \mu}, V\right]=1$. Then $V=1$ unless $p=2, \mu={ }^{2} \sigma_{q^{r} / 2}, \lambda=\sigma_{q}$, $G=A_{n}(k), n$ even, and $|V| \mid q+1$ and $O^{p^{\prime}}\left(C_{G_{\mu}}(V)\right) / Z\left(O^{p^{\prime}}\left(C_{G_{\mu}}(V)\right)\right) \cong$ ${ }^{2} A_{n-1}(q)$.

Proof. If $G_{\mu}$ has rank 1, i.e., $G_{\mu} \cong A_{1}(q),{ }^{2} A_{2}(q),{ }^{2} C_{2}(q)$ or ${ }^{2} G_{2}(q)$, the lemma is well-known to be true.

Let $G$ be a counterexample of minimal rank. Letting $\Pi$ be the set of fundamental roots, we may apply induction to $\bar{P}=P / O_{p}(P), P$ any parabolic subgroup. Then $\bar{V} \leqq Z(\bar{P})$ unless $\bar{P} / Z(\bar{P})$ has a component of type $A_{l}, l$ even. If $\bar{V} \leqq Z(\bar{P})$, the Frattini argument shows $C_{G}(V)$ covers $P / O_{p}(P)$. Since $V \neq 1, C_{G}(V)$ cannot cover all such $P / O_{p}(P)$, whence $G$ has type $A_{n}, n$ even. On the other hand, letting $P$ be associated with various subsets of $\Pi$, we see that $V$ centralizes all root groups, for short roots in $\Sigma_{\mu}$, and on any root group for a long root in $\Sigma_{\mu}, V$ centralizes precisely the center. The remaining statements now follow.

Lemma 3.8. Let $P$ be a proper parabolic subgroup of $G$ containing $B$. Assume $P$ is $\langle\lambda, \mu\rangle$-invariant. Then $C_{P_{\mu}}\left(O_{p}\left(P_{\lambda, \mu}\right)\right) \leqq$ $O_{p}\left(P_{\mu}\right) \cdot K$ where $K=1$ unless $G_{\mu}={ }^{2} A_{n}(q), n, q$ even and $K \leqq H$ is a cyclic group of order dividing $q+1$ and centralizing $G_{\lambda, \mu \cdot}$. In particular, $C_{G_{\mu}}\left(G_{\lambda, \mu}\right)=1$ unless $G_{\mu}={ }^{2} A_{n}(q), n, q$ even, and $G_{\lambda, \mu} \cong$ $C_{n / 2}(q)$, in which case $C_{G_{\mu}}\left(G_{\lambda, \mu}\right) \cong Z_{q+1}$.

Proof. The last sentence follows from the first statement of the lemma whose proof we now begin. We may assume $r$ is a prime and that $r=s$ if there is a graph automorphism involved in $\mu$. Let 
$C=C_{P}\left(O_{p}\left(P_{\lambda, \mu}\right)\right)$ and let - be the quotient map $P \rightarrow \bar{P}=P / O_{p}(P)$. We may assume $\bar{C} \neq 1$. Since $\bar{C} \neq 1, P \neq B$, and so $G_{\mu}$ has rank at least 2. Let $L$ be the standard $\langle\lambda, \mu\rangle$-invariant complemet to $O_{p}(P)$ in $P$ (i.e., $L=\left\langle H, X_{\alpha}\right| \alpha$ runs over a subset of $\left.\Sigma\right\rangle$ ). Then $\bar{P} \cong L$ as $\langle\lambda, \mu\rangle$-groups. Since $L_{\lambda, \mu}$ normalizes $O_{q}\left(P_{\lambda, \mu}\right), L_{\lambda, \mu}$ normalizes $D=$ $C \cap L \cong \bar{C}$.

Assume that $D_{0}=C_{D}\left(O^{p^{\prime}}\left(L_{\lambda, \mu}\right)\right)=C_{D}\left(O^{p^{\prime}}\left(P_{\lambda, \mu}\right)\right) \neq 1$. A Frattini argument then shows $D_{0}$ centralizes $O_{p}\left(P_{\lambda, \mu}\right)\left(U \cap L_{\lambda, \mu}\right)=U_{\lambda, \mu}$. By Lemma $3.7 G_{\mu} \cong{ }^{2} A_{n}(q), n, q$ even, and $1 \neq D_{0} \leqq K$ in the notation of Lemma 3.7. Then, as $D_{0} \leqq D, D \leqq N_{G_{\mu}}(K)$ and the lemma is verified by inspection.

We may now assume $D_{0}=1$. This will eventually lead to a contradiction. Now $D_{\lambda} \leqq C_{P_{\lambda}}\left(O_{p}\left(P_{\lambda}\right)\right) \leqq O_{p}\left(P_{\lambda}\right)$, by Lemma 2. So, $D_{\lambda}=1$. We may assume $D_{\mu} \neq 1$. Since $r$ is prime, $D_{\mu}$ is nilpotent by Thompson's theorem [13]. Let $1 \neq V \leqq D_{\mu}$ be minimal normal in $D_{\mu} L_{\lambda, \mu}\langle\lambda\rangle$. Then $V$ is an elementary abelian $t$-group, for some prime $t \neq r$.

Assume that $t=p$. Let $L_{1}, \cdots, L_{n}$ be the components of $O^{p^{\prime}}\left(L_{\mu}\right)$ and let $\pi_{i}: O^{p^{\prime}}\left(L_{\mu}\right) \rightarrow \bar{L}_{i}=L_{i} / Z\left(L_{i}\right)$ be the "projections." Our hypotheses on $\lambda, \mu$ imply that $\lambda$ stabilizes each $L_{i}$. Since $V \neq 1$ is a $p$ group, and $Z\left(L_{i}\right)$ is a $p^{\prime}$-group for all $i, V^{\pi_{i}} \neq 1$ for some $i$. Then $V^{\pi_{i}}\left(\bar{L}_{i}\right)_{\lambda}$ lies in a proper parabolic subgroup of $\bar{L}_{i}$, which is impossible by Lemma 3.5. Thus $t \neq p$.

Take $S \leqq O_{p}\left(P_{\mu}\right)$ such that $S>O_{p}\left(P_{\lambda, \mu}\right)=S_{\lambda, \mu}, S_{\lambda} \leqq C_{S}(V) \triangleleft S$ and $S / C_{S}(V)$ is an irreducible $V\langle\lambda\rangle$-module for which $C_{V}(S)<V$ (such a choice is possible because $O_{p}\left(P_{\mu}\right)>O_{p}\left(P_{\lambda, \mu}\right), t \neq p, V \leqq P_{\mu}$ and $\left.O_{p}\left(P_{\mu}\right) \geqq C_{P_{\mu}}\left(O_{p}\left(P_{\mu}\right)\right)\right)$.

We claim that $r=p$. If $r \neq p$, then $\left(S / C_{S}(V)\right)_{2}=1$, which implies $S V / C_{S}(V)$ is nilpotent, whence $[S, V] \leqq C_{S}(V),[S, V]=[S, V, V]=1$ and so $S \leqq C_{S}(V)$, which is false. Therefore $r=p$.

We next argue that $p=2$. In $S$, take a minimal $V\langle\lambda\rangle$-invariant subgroup $T$ which covers $S / C_{S}(V)$. Then $T$ is special or elementary abelian, $T=[T, V]$ and $C_{T}(V)=T^{\prime}$. Since $V\langle\lambda\rangle /\left\langle\lambda^{p}\right\rangle$ is a Frobenius group, $S / \dot{C}_{S}(V) \cong T / C_{T}(V)$ is a free $A=F_{p}\left(\langle\lambda\rangle /\left\langle\lambda^{p}\right\rangle\right)$-module. Choose $T_{1} \leqq T$ so that $T_{1} \geqq C_{T}(V), T_{1} / C_{T}(V)$ has order $p^{p}$ and is a free $\Lambda$ module. Observe that $T_{1}$ cannot be elementary, or else $t \neq p$ implies that $T_{1} \cong C_{T}(V) \times T_{1} / C_{T}(V)$ as $\langle\lambda\rangle$-groups, and freeness of the right factor over $\Lambda$ contradicts $\left(T_{1}\right)_{\lambda} \leqq C_{T}(V)$. Take any hyperplane $A$ of $C_{T}(V)$ which is $\lambda$-invariant. Then $T_{1}\left(\langle\lambda\rangle /\left\langle\lambda^{p}\right\rangle\right)$ is a "maximal group of maximal class," so by one of [26], [7], [3] we get, for odd $p$, $\left.Z\left(T_{1}\left(\langle\lambda\rangle /\left\langle\lambda^{p}\right\rangle\right)\right) / A\right\rangle C_{T}(V) / A$. So assume $p$ odd. Since $T / C_{T}(V)$ is an irreducible $V\langle\lambda\rangle$-module, and since $Z(T / A)>C_{T}(V) / A$, it follows that $T / A$ is abelian, hence $T=[T, V] \times C_{T}(A)=[T, V]$ is elementary, which is impossible as noted above. Therefore, $p=2$ and we also 
get $\mathrm{O}_{2}\left(P_{\mu}\right)$ nonabelian.

Next consider the action of involutions in $L_{\lambda, \mu}$ on $V$. Suppose there is an involution $w$ in $L_{\lambda, \mu}$ with $C_{V}(w) \neq 1$. Then $C_{L_{\lambda, \mu}}(w) \leqq$ $Q$, a proper parabolic subgroup of $L_{\lambda, \mu}$. Let $Q_{1}=O_{2}(Q), Q_{0}=C_{Q_{1}}(w)$. Then we get $\left[C_{V}(w), Q_{0}\right] \leqq Q_{0} \cap C_{V}(w)=1$ (because $L_{\lambda, \mu}$ normalizes $V$ ). So, $\left[C_{V}(w), Q_{1}\right]=1$, by the $P \times Q$ lemma. By induction and $t \neq 2$, we get that $V \cap L_{i} \leqq Z\left(L_{i}\right)$ whenever $L_{i}$ is a component of $L_{\mu}$ such that $w \notin C\left(L_{i}\right)$.

If $\left[L_{i}, w\right]=1$, we claim that $V^{\pi_{i}}=1$. Suppose $i$ is an index for which $\left[L_{i}, w\right]=1$ and $V^{\pi_{i}} \neq 1$. Set $Y=L_{i}$. Then $V^{\tau_{i}}$ is normalized by $Y_{\lambda}$. If, for some involution $x$ in the center of a Sylow group of $Y_{\lambda}, C_{V} \pi_{i}(x) \neq 1$, we apply induction to get a contradiction. Therefore, by easy calculation, one concludes that there is no four-group $W$ in $Y_{\lambda}$. Therefore $Y_{\lambda} \cong A_{1}(2),{ }^{2} A_{2}(2),{ }^{2} B_{2}(2)$.

We eliminate these cases. First assume $Y_{\lambda} \cong A_{1}(2)$. Then $Y \cong$ $A_{1}(4)$ or ${ }^{2} A_{2}(2)$. But $Y \cong A_{1}(4)$ is out because the only possibility for $V^{\pi_{i}}$ is $O_{3}\left(Y_{\lambda}\right)$, whence $V^{\pi_{i}} \cong\left[V, Y_{\lambda}\right] \leqq V$. The $P \times Q$ lemma applied to the action of $\left(\langle\lambda\rangle /\left\langle\lambda^{2}\right\rangle\right) \times\left[V, Y_{\lambda}\right]$ on $O_{2}\left(P_{\mu}\right)$ tells us that $\left[V, Y_{\lambda}\right]$ centralizes $O_{2}\left(P_{\mu}\right)$, against Lemma 3.3. Thus $Y \cong{ }^{2} A_{2}(2)$ and $Y_{\lambda} \cong A_{1}(2)$. Also, $G_{\mu} \cong{ }^{2} A_{2 m}(2)$, and $m \geqq 3$, since $w \in L$ centralizes $Y_{\lambda}$. The only possiblity is $\left|V^{\pi_{i}}\right|=3$. Since $V$ is an irreducible $\langle\lambda\rangle$-module, $V^{\pi_{i}} \cong$ $\left[V, Y_{\lambda}\right]$. We have $V_{\lambda}^{\pi_{i}}=1$ because $D_{\lambda}=1$. Thus, as $\left[V, Y_{\lambda}\right]$ is cyclic and is normalized by $Y_{\lambda}$, the structure of $P S U(3,2)$ implies $Z(Y)=1$. Now it is clear that the parabolic subgroup $P$ we are considering is associated with a subset of the Dynkin diagram

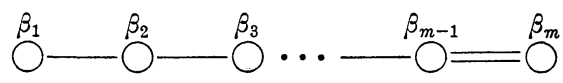

for $G_{\mu}$ (type $C_{m}, m \geqq 3$ ) which contains the rightmost (long) root, $\beta_{m}$, but not $\beta_{m-1}$. Let $Q$ be the parabolic subgroup associated with $\left\{\beta_{2}, \beta_{3}, \cdots, \beta_{m}\right\}$. Then $O^{\prime \prime}(Q) / O_{2}(Q) \cong S U(2 m-1,2)$ and $O_{2}(Q)$ is the "standard module" for $S U(2 m-1,2)$. In particular, as $Y$ is the group generated by the root groups associated with $\pm \beta_{m}, Y \cong$ $S U(3,2)$. But this contradicts $Z(Y)=1$. Thus, $Y_{\lambda} \cong A_{1}(2)$ is impossible.

Suppose $Y_{\lambda} \cong{ }^{2} A_{2}(2)$. Since $r=2$ one sees that $\lambda$ cannot induce a field automorphism on $Y$ by inspecting the possibilities. Thus $\lambda=$ ${ }^{s} \sigma_{q}, s \in\{2,3\}$. If $\mu=\lambda^{2}$ were not a field automorphism, $s=3$ and $\lambda$ would induce a field automorphism on $Y$, which is impossible. Thus $s=2$ and $\mu=\lambda^{2}$ is a field automorphism; in fact $\lambda={ }^{2} \sigma_{2}, \mu=\sigma_{4}, Y \cong$ $A_{2}(4)$. Then, the structure of $A_{2}(4)$ and $\left[V, Y_{\lambda}\right] \neq 1$ implies that $\left[V, Y_{\lambda}\right]=Z(Y) \cong Z_{3}$. But then $V=\left[V, Y_{\lambda}\right]$ cannot satisfy $V^{\pi_{i}} \neq 1$, contradiction.

Suppose $Y_{\lambda} \cong{ }^{2} B_{2}(2)$. Then $r=2$ implies that $Y$ is not of type 
${ }^{2} B_{2}$. Thus, $Y \cong B_{2}(2)$. Clearly, $V^{\pi_{i}} \cong 1$ and $V_{\lambda}=1$ are impossible in this case.

We conclude that each $V^{\pi_{i}}=1$, i.e., that $V \cap O^{2^{\prime}}\left(L_{\mu}\right) \leqq Z\left(O^{2^{\prime}}\left(L_{\mu}\right)\right) \leqq$ $H_{\mu}$. Therefore, $\left[V, L \cap U_{\lambda, \mu}\right] \leqq H_{\mu} \cap V$. Since $t \neq p,\left[V, L \cap U_{\lambda, \mu}\right]=$ $\left[V, L \cap U_{\lambda, \mu}, L \cap U_{\lambda, \mu}\right] \leqq\left[H_{\mu}, U_{\lambda, \mu}\right] \leqq U$. Therefore $\left[V, L \cap U_{\lambda, \mu}\right]=1$. Since $\left[O_{2}(P)_{\lambda, \mu}, V\right]=1$, this gives $\left[V, U_{\lambda, \mu}\right]=1$. We new quote Lemma 3.7 to see that our lemma holds.

It therefore remains to treat the case that $C_{V}(w)=1$ for every involution $w$ in $L_{\lambda, \mu}$. Assume this. If $W \leqq L_{\lambda, \mu}$ is elementary of order $4, V=\left\langle C_{V}(x) \mid x \in W^{\sharp}\right\rangle$. So, no such $W$ exist, i.e., $L_{\lambda, \mu}$ has cyclic or quaternion Sylow 2-groups. Thus $r=2$ implies that $L_{\mu} \cong A_{1}(4)$ or ${ }^{2} A_{2}(2)$ if $L_{\mu}>L_{\lambda, \mu}$ and $L_{\mu}=A_{1}(2)$ or ${ }^{2} A_{2}(2)$ if $L_{\mu}=L_{\lambda, \mu}$.

At this point we may enlarge $P$ if necessary to assume that $P_{\mu}$ is a maximal parabolic subgroup of $G_{\mu}$. Thus, $G_{\mu}$ has rank 2 . If $L_{\mu} \cong A_{1}(4)$, then $G_{\mu} \cong A_{2}(4), B_{2}(4),{ }^{2} A_{3}(2),{ }^{2} A_{3}(4)$ or ${ }^{2} A_{4}(2)$. If $L_{\mu} \cong{ }^{2} A_{2}(2)$, then $G_{\mu} \cong{ }^{2} A_{4}(2)$. If $L_{\mu} \cong A_{1}(2)$, then $G_{\mu} \cong{ }^{2} A_{3}(2)$. By inspection, each of these groups satisfies the conclusion of the lemma, so that the proof is complete.

Proposition 3.1. Let $M$ be a group such that $O^{p^{\prime}}\left(G_{\lambda, \mu}\right) \leqq M<G_{\mu}$, $M \not G_{\lambda, \mu}$ and $U_{\lambda, \mu} \in \operatorname{Syl}_{p}(M)$. Then $\widetilde{M}_{\lambda, \mu}=N_{M}\left(O^{p^{\prime}}\left(G_{\lambda, \mu}\right)\right)$ is strongly p-embedded in $M$.

(Note that $G_{\lambda, \mu}=N_{G}\left(G_{\lambda, \mu}\right)$ unless $G=A_{n}(k), n, q$ even, $\mu=$ ${ }^{2} \sigma_{q^{r / s}}, \lambda=\sigma_{q}$.)

Proof. Let $R \neq 1$ be a $p$-group in $G_{\lambda, \mu}$ and, as in Lemma 3.4 embed $N_{G_{\lambda, \mu}}(R)$ in $P(\lambda, \mu)$, a parabolic subgroup of $G_{\lambda, \mu}$. We may assume that $P(\lambda, \mu) \geqq U_{\lambda, \mu}$ by replacing $R$ with a conjugate by an element of $O^{p^{\prime}}\left(G_{\lambda, \mu}\right)$ if necessary. Using Lemma 3.4(iv), we have that $P(\lambda, \mu)$ lies in a unique parabolic subgroup $P(\mu)$ of $G_{\mu}$ with $P(\mu)_{\lambda}=$ $P(\lambda, \mu)$. By Lemma 3.4(v), we may take $P$, a $\langle\lambda, \mu\rangle$-invariant parabolic subgroup of $G$ with $P_{\mu}=P(\mu)$ and we may assume $U \leqq P$, by Lemma 3.4(i).

It suffices to prove $M \cap P=M \cap P_{\mu} \leqq P_{\lambda, \mu} \cdot K$, where $K$ is as in Lemma 3.8. Set $C=C_{P_{\mu}}\left(O_{p}\left(P_{\lambda, \mu}\right)\right)$ and take $g \in M \cap P_{\mu}$. Then $U_{\lambda, \mu} \in$ $\operatorname{Syl}_{p}(M)$ implies that $M \cap P_{\mu}$ normalizes $O_{p}\left(P_{\lambda, \mu}\right)$, whence $\left[g, O_{p}\left(P_{\lambda, \mu}\right), \lambda\right]=$ 1. Clearly $\left[O_{p}\left(P_{\lambda, \mu}\right), \lambda, g\right]=1$, and so $\left[\lambda, g, O_{p}\left(P_{\lambda, \mu}\right)\right]=1$ by the three subgroups lemma, Thus $[\lambda, g] \in C$. By Lemma $3.8 C \leqq O_{p}\left(P_{\mu}\right) \cdot K$, where $K \leqq H_{\mu},|K| \mid q+1$. Letting - be the quotient $P \rightarrow \bar{P}=$ $P / O_{p}(P)$, we get $[\overline{P \cap M}, \lambda] \leqq \bar{C}=\bar{K}$. Thus $\overline{P \cap M} \leqq \bar{P}_{\lambda, \mu}$ or if $\bar{K} \neq 1$, $\overline{P \cap M} \leqq N_{\bar{P}_{\mu}}([\overline{P \cap M}, \lambda]) \leqq N_{\bar{P}_{\mu}}(\bar{K})=C_{\bar{P}_{\mu}}(\bar{K})$ and $\bar{P}$ has a component of type $A_{n}(k), n, q$ even. Also, we may enlarge $P$, if necessary, to assume that $\bar{P}_{\mu}$ has one component.

Suppose $\overline{P \cap M} \leqq \bar{P}_{\lambda, \mu}$. Then $O^{2^{\prime}}\left(P_{\lambda, \mu}\right) \leqq P \cap M \leqq O_{2}\left(P_{\mu}\right) \cdot L_{\lambda, \mu}$, where 
$L$ is a $\langle\lambda, \mu\rangle$-invariant complement to $O_{2}(P)$ in $P$. Then $\left(\left|M: G_{\lambda, \mu}\right|, 2\right)=$ 1 implies that $P \cap M=O^{2^{\prime}}\left(P_{\lambda, \mu}\right)$, as required. Thus, we may suppose $\overline{P \cap M} \geqq \bar{P}_{\lambda, \mu}$. Let $K, L$ be as above. We have $1 \neq[\overline{P \cap M}, \lambda] \leqq \bar{K}$, $q$ is even and $G=A_{n}(k), n$ even, $\mu=2_{\sigma_{q} / 2}, \lambda=\sigma_{q}$. From Lemma 3.8, we know that $\left.O^{2^{\prime}}\left(C_{\bar{P}_{u}}(\bar{K})\right) / Z\left(O^{2^{\prime}} C_{\bar{P}_{\mu}}(\bar{K})\right)\right) \cong{ }^{2} A_{n-1}(q)$. Thus $\bar{Y}=$ $O^{2^{\prime}}\left(C_{\bar{P}_{\mu}}(\bar{K})\right)$ satisfies: $\overline{P \cap M} \cap \bar{Y}$ contains a Sylow 2-group of $\overline{P \cap M}$. Since $\bar{U}_{\mu, \lambda} \leqq O^{2}\left(\bar{Y}_{\lambda}\right) \leqq O^{2}(\overline{P \cap M})$, we may apply induction to $\bar{P}$ to get $O^{2^{\prime}}\left(\bar{Y}_{\lambda}\right) \cong C_{n / 2}(q)$. The structure of $\bar{P}_{\mu}$ implies that $N_{\bar{P}_{\mu}}\left(\bar{Y}_{\lambda}\right)=$ $\bar{K} \times \bar{Y}_{\lambda}$, whence $\overline{P \cap M}=(\overline{P \cap M} \cap \bar{K}) \times \bar{Y}_{\lambda}$.

As in the case $\overline{P \cap M} \leqq \bar{P}_{\lambda, \mu}$, we argue that $O^{2^{\prime}}\left(P_{\lambda, \mu}\right)=O^{2^{\prime}}(P \cap M)$. Write $\left(O_{2}\left(P_{\mu}\right) \cdot K\right) \cap M=O_{2}\left(P_{\lambda, \mu}\right) \cdot K_{1}$, where $K_{1}$ is a cyclic $2^{\prime}$-group. Now, $K_{1}$ is trivial on the Frattini factor group of $O_{2}\left(P_{\lambda, \mu}\right)$, because $K$ is, whence $K_{1}$ centralizes $O_{2}\left(P_{\lambda, \mu}\right)$. But also, [ $\left.U_{\lambda, \mu}, K_{1}\right] \leqq O_{2}\left(P_{\lambda, \mu}\right)$. Since $K_{1}$ then stabilizes the chain $U_{\lambda, \mu} \geqq O_{2}\left(P_{\lambda, \mu}\right) \geqq 1$, we get $K_{1} \leqq$ $C\left(U_{\lambda, \mu}\right)$. The Frattini argument on $O_{2}\left(P_{\lambda, \mu}\right) K_{1} \triangleleft P \cap M$ implies that $C_{P \cap M}\left(K_{1}\right)$ covers $\overline{P \cap M}$, whence $K_{1} \leqq Z(P \cap M)$. Since $K$ contains a Hall 2 -subgroup of $Z(P \cap M)$, it follows that $K_{1} \leqq K$, whence $K_{1}=$ $K \cap M$. Therefore, $M \leqq P_{\lambda, \mu} \cdot K$, as required.

COROLLARY. If $p=2,|M|_{2}=\left|U_{\lambda, \mu}\right|, M \geqq O^{2^{\prime}}\left(G_{\lambda, \mu}\right)$ and $M \geqq G_{\lambda, \mu}$, then $\mu \in\langle\lambda\rangle$ and $M$ lies in a unique maximal subgroup $M_{0}$ of $G_{\mu}$, and we are in one of the following situations.

(a) $G_{\lambda} \cong A_{1}(2), M_{0} \cong D_{2^{r+1}}$, and $r$ is odd, $r \geqq 3 ; G_{\mu} \cong A_{1}\left(2^{r}\right)$

(b) $G_{\lambda} \cong{ }^{2} B_{2}(2) \cong S z(2), r$ is odd, $r \geqq 5$, and $M_{0}$ is a Frobenius group of order

$$
4\left(2^{r} \pm 2^{(r+1) / 2}+1\right) ; G_{\mu} \cong{ }^{2} B_{2}\left(2^{r}\right) .
$$

Proof. Let $L=O^{2}\left(G_{\lambda, \mu}\right)$ then $\tilde{M}_{\lambda, \mu}=N_{M}\left(O^{2}\left(G_{\lambda, \mu}\right)\right)$ is strongly embedded in $M$ and $L=O_{2^{\prime}, 2}(L)$, which implies $L \cong A_{1}(2),{ }^{2} B_{2}(2)$ or ${ }^{2} A_{2}(2)$. We claim that $L \cong{ }^{2} A_{2}(2)$ is impossible. So, assume $L \cong{ }^{2} A_{2}(2)$. Then $G_{\mu}$ must be isomorphic to ${ }^{2} A_{2}\left(2^{r}\right)$ for odd $r \geqq 3$. Let $t$ be an involution of $L$. Then $t$ inverts $O(M)$ because $C_{G_{u}}(t)=$ $U_{\mu}$. Thus, $O(L)=[O(L), t] \leqq O(M)$. An easy calculation (which we omit) shows that $O(L) \cong Z_{3} \times Z_{3}$ is self centralizing in $G_{\mu}$. This means $O(L)=O(M)$ and so $M \leqq N_{G_{\mu}}(O(L))=G_{\lambda, \mu} \cong P G U(3,2)$, i.e., we have no exception in this case. Therefore, $M$ has cyclic Sylow 2-groups, whence $M=O_{2^{\prime}, 2}(M)$. A survey of the possibilities produces (a) and (b) as the precise list of exceptions to $M \nsubseteq G_{\lambda, \mu}$.

REMARK. We henceforth assume that $p$ is odd. Thus, $\widetilde{M}_{\lambda, \mu}=$ $M_{\lambda, \mu}=M \cap G_{\lambda, \mu}$ (see Lemma 3.8 and use $G_{\lambda, \mu}=N_{G_{\mu}}\left(O^{p^{\prime}}\left(G_{\lambda, \mu}\right)\right.$ ) if $G_{\mu} \not$ ${ }^{2} A_{n}(q), n, q$ even).

LEMMA 3.9. If $t$ is an involution of $M_{\lambda, \mu}$, then $C_{M}(t) \leqq M_{\lambda, \mu}$ 
unless either $\lambda^{r}=\mu$ (i.e., Theorem 1 applies to $G$ ) or one of (2), (3), (5), (6) holds.

Proof. Let $t$ be an involution of $M_{\lambda, \mu}$. Set $C=C_{G}(t)$. Then $C=$ $\widetilde{H} L$, where $\widetilde{H}$ is a conjugate of $H$ and $L=O^{p^{\prime}}(C)$.

We assume that $C \cap M \not M_{\lambda, \mu}$.

Case 1. $L=1$. Then, letting $t^{\prime}$ be a conjugate of $t$ in $H$, have that $t^{\prime}$ inverts every $X_{\alpha}, \alpha \in \Sigma$. This implies that $U$ is abelian, so that $G=A_{1}(k)$. Thus, $\mu=\lambda^{r}$ and Theorem 1 applies.

We observe that, if $L$ contains some $\tilde{L} \triangleleft C$ with $p|| \widetilde{L}_{\lambda, \mu} \mid$ and $\tilde{L} \cap M=\widetilde{L}_{\lambda, \mu}$, we are done; for then, letting $R \in \operatorname{Syl}_{p}(\tilde{L} \cap M)$ we have $M=(\tilde{L} \cap M) \cdot N_{M}(R) \leqq M_{\lambda, \mu}$, a contradiction.

Case 2. $L \neq 1$ and quasisimple of rank at least 2. Then by induction, $C \cap M \leqq M_{\lambda, \mu}$ unless $L_{\mu} / Z\left(L_{\mu}\right) \cong{ }^{2} A_{2}(p), p=3$ or 5 . In the latter case, $L / Z(L) \cong A_{2}(k)$. Let $t^{\prime}$ be a conjugate of $t$ in $H$ and let $X_{\alpha}, X_{\beta}, X_{\alpha+\beta}$ be the root groups centralized by $t^{\prime}$. The shape of $L_{\mu}$ forces $G=A_{n}(k), n \geqq 4$ and $\mu={ }^{2} \sigma_{p}$. Since $n \geqq 4$, we may choose roots $\gamma$ and $\delta$ so that $\{\alpha, \beta, \gamma, \delta\}$ is a linearly independent set such that $\gamma+\delta$ is a root. Then, as $t^{\prime}$ inverts $X_{r}$ and $X_{r}, t^{\prime}$ centralizes $X_{\gamma+\delta}=\left[X_{\gamma}, X_{\delta}\right]$. Since $\gamma+\delta$ is not in the span of $\alpha$ and $\beta$, this is a contradiction. Thus, Case 2 does not hold.

Case 3. $L \neq 1$ and quasisimple of $\operatorname{rank} 1$, i.e., $L / Z(L) \cong A_{1}(k)$. Let $t^{\prime}$ be a conjugate of $t$ in $H$. Then $t^{\prime}$ inverts $X_{\beta}$ for all $\beta \neq \alpha$, $\alpha$ a fixed root in $\Sigma^{+}$(as in Case 1 , we know $U$ is nonabelian). It follows that $C_{G}\left(X_{\alpha}\right) / X_{\alpha}$ has abelian Sylow $p$-subgroups. Also, if $O^{p^{\prime}}\left(C_{G}\left(X_{\alpha}\right) / X_{\alpha}\right.$ were strictly larger then $O_{p}\left(C_{G}\left(X_{\alpha}\right) / X_{\alpha}\right)$, a Frattini argument would show that $t^{\prime}$ centralize some $X_{\beta}, \beta \neq \alpha$. Since this is false, $O^{p^{\prime}}\left(C_{G}\left(X_{\alpha}\right) / X_{\alpha}\right)=O_{p}\left(C_{G}\left(X_{\alpha}\right) / X_{\alpha}\right)$. Therefore, if $\alpha$ is long, $G=$ $A_{2}(k)$ and if $\alpha$ is short, the fact that there are no long roots orthogonal to $\alpha$ implies $G=B_{2}(k)$.

Assume $G=B_{2}(k)$. Then $\langle\lambda, \mu\rangle$ is a cyclic group and Theorem 1 applies since $G_{\lambda, \mu}$ is not an exceptional case.

Thus $G=A_{2}(k)$. If $\langle\lambda, \mu\rangle$ is cyclic, then Theorem 1 applies since $G_{\lambda, \mu}$ cannot be an exceptional case. So we may assume $\langle\lambda, \mu\rangle$ is not cyclic. We then have $\mu={ }^{2} \sigma_{q} r / 2$ and $\lambda=\sigma_{q}$. Then $G_{\lambda, \mu} \cong P G L(2, q)$ and we quote [22] to get that (2), (3), (5) or (6) holds.

Case 4. $L \neq 1$ is not quasisimple. Let $\widetilde{L} \not Z Z(L)$ be any $\langle\lambda, \mu\rangle$ invariant normal subgroup of $L$. By Lemma 3.2 we have that $\left|\widetilde{L}_{\lambda, \mu}\right| \equiv$ $0(\bmod p)$. Thus, if $\langle\lambda, \mu\rangle$ had more than one orbit on the set of components of $L$, Lemma 3.8 applied to an $\widetilde{L}$ as above, $\widetilde{L} \neq L$ dan 
to $C_{L}(\tilde{L}) \neq 1$, shows that $L \cap M=M_{\lambda, \mu}$, a contradiction. Therefore, $\langle\lambda, \mu\rangle$ has one orbit on the set of components of $L$. So, $L$ has $s \in$ $\{2,3\}$ components, $\langle\mu\rangle$ is transitive on them and $\lambda$ normalizes each one.

Since $L \cap M>L_{\lambda, \mu}$, induction implies that $O^{p^{\prime}}\left(L_{\lambda, \mu}\right) / Z\left(L_{\lambda, \mu}\right) \cong A_{1}(3)$, $A_{1}(5)$, or $A_{1}(5)$ and $L \cap M \cong A_{5}, A_{7}$ or $M_{10}$ respectively. But then $L_{\mu} / Z\left(L_{\mu}\right)$ must be isomorphic to, respectively, $A_{1}(9),{ }^{2} A_{2}(5)$ or ${ }^{2} A_{2}(5)$. No $\mu$ of the form ${ }^{8} \sigma_{q} r / s$ will give $L_{\mu} / Z\left(L_{\mu}\right)$ isomorphic to any of these possibilities. This final contradiction proves the lemma.

Proposition 3.2. Suppose $M_{\lambda, \mu}<M$. Then $M_{\lambda, \mu}$ is strongly embedded in $M$, or else (6) or an exceptional case listed in (2.2) holds.

Proof. By Lemma 3.9, it suffices to prove that $N_{M}(S) \leqq M_{\lambda, \mu}$, for $S \in \operatorname{Syl}_{2}\left(M_{\lambda, \mu}\right)$. Supposing this to be false, take an element $g \in N_{M}(S)-M_{\lambda, \mu}$ of odd order such that $\langle g\rangle$ causes fusion among elements of $Z \leqq \Omega_{1}(Z(S))$ which are not fused in $M$. Let $z_{1}, z_{2}$ be two such elements. Assume that $\left|C_{M_{\lambda, \mu}}\left(z_{1}\right)\right| \equiv 0(\bmod p), i=1,2$. Then, as $O^{p^{\prime}}\left(C_{M \lambda, \mu}\left(z_{1}\right)\right)$ and $O^{p^{\prime}}\left(C_{M_{\lambda, \mu}}\left(z_{2}\right)\right)$ are fused under $g,\left|M_{\lambda, \mu} \cap M_{\lambda, \mu}^{g}\right| \equiv$ $0(\bmod p)$. By Proposition 3.1, this forces $g \in M_{\lambda, \mu}$, contradiction. Hence we must show that $\left|C_{M_{\lambda, \mu}}\left(z_{i}\right)\right| \equiv 0(\bmod p)$.

The arguments in the proof of Lemma 3.9 show that if $O^{p^{\prime}}\left(C_{G}\left(z_{i}\right)\right) \neq$ 1 , then $\left.O^{p^{\prime}} C_{G_{\lambda, \mu}}\left(z_{i}\right)\right) \neq 1$, so that we may assume $O^{p^{\prime}}\left(C_{G}\left(z_{i}\right)\right)=1$. Then, as in Case 1 in the proof of Lemma 3.9, we get that $G=A_{1}(k)$. But then $\langle\lambda, \mu\rangle$ is cyclic, and Theorem 1 tells us that $p=3, G_{\mu} \cong A_{1}(9)$ and $M \cong \Sigma_{5}$ as in (2.2).

LEMma 3.10. $G, \mu, \lambda$ and $M$ satisfy one of the conclusions of Theorem 2.

Proof. If false, Proposition 3.2 tells us that $M_{\lambda, \mu}$ is strongly embedded in $M$. By Bender's theorem [2] and Theorem 1, as $\langle\lambda, \mu\rangle$ is not cyclic, $M_{\lambda, \mu}$ is a solvable Steinberg variation. The only possibility is ${ }^{2} A_{2}(2)$, where $p=2$ and and the Corollary to Proposition 3.1 tells us that no such $M$ exists, contradiction.

This completes the proof of Theorem 2.

\section{REFERENCES}

1. E. Artin, Geometric Algebra, Interscience, N. Y. (1957).

2. H. Bender, Transitive Gruppen gerader Ordnung, in denen jede Involution genau einen Punkt festläßt, J. Algebra, 17 (1971), 527-554.

3. N. Blackburn, On a special class of p-groups, Acta Math., 100 (1958), 45-93.

4. A. Borel and J. Tits, Éléments unipotents et sous-groupes paraboliques de groupes 
réductifs. I, Inv. Math., 12 (1971), 95-105.

5. R. Carter, Simple Groups of Lie Type, J. Wiley \& Sons, N. Y. (1972).

6. L. Dickson, Linear Groups, Dover, N. Y. (1958).

7. L. Evans, On a theorem of Thompson on fixed points of p-groups acting on p-groups, Math. Z., 93 (1966), 105-8.

8. W. Feit and J. Thompson, Solvability of groups of odd order, Pacific J. Math., 13 (1963), 775-1029.

9. B. Fischer, Finite groups generated by 3-transpositions. I, Inv. Math., 13 (1971), 232-246.

10. - Subgroups of ${ }^{2} E_{6}(2)$ generated by 3-transpositions, (to appear).

11. D. E. Flesner, Maximal subgroups of $\mathrm{PSp}_{4}\left(2^{n}\right)$ containing central elations or noncentered skew elations, Illinois J. Math., 19 (1975), 247-268.

12. G. Glauberman, Central elements in core free groups, J. Algebra, 4 (1966), 403-420.

13. D. Gorenstein, Finite Groups, Harper \& Row, N. Y. (1968).

14. D. Gorenstein and K. Harada, Finite groups whose 2-subgroups are generated by at most 4 elements, Mem. Amer. Math. Soc., No. 147 (1974).

15. R. Griess, Schur multipliers of finite simple groups of Lie type, Trans. Amer. Math. Soc., 183 (1973), 355-421.

16. R. W. Hartley, Determination of the ternary collineation groups whose coefficients Lie in the $G F\left(2^{n}\right)$, Ann. Math., 27 (1925), 140-158.

17. J. Humphreys, Linear Algebraic Groups, Springer-Verlag, N. Y. (1975).

18. N. Iwahori, Centralizers of involutions in finite Chevalley group, Lecture Notes in Math. No. 131, Springer-Verlag (1970).

19. Z. Janko, A new finite simple group with abelian Sylow 2-subgroups and its characterization, J. Algebra, 4 (1966), 147-186.

20. S. Lang, Algebraic groups over finite fields, Amer. J. Math., 78 (1956), 555-563.

21. J. McLaughlin, A simple group of order $898,128,000$, From R. Brauer \& H. Sah, 'Theory of Finite Groups', Benjamin, N. Y. (1969).

22. H. N. Mitchell, Determination of the ordinary and modular ternary linear groups, Trans. Amer. Math. Soc., 12 (1911), 207-242.

23. R. Steinberg, Endomorphisms of linear algebraic groups, Mem. Amer. Math. Soc., no. 80 (1968).

24. - Lectures on Chevalley Groups, Yale Univ. (1967).

25. M. Suzuki, On a class of doubly transitive groups, Ann. Math., 75 (1962), 105-145.

26. J. Thompson, Fixed points of p-groups acting on p-groups, Math. Z., 86 (1964), $12-13$.

27. D. Wales, Generators of the Hall-Janko group as a subgroup of $G_{2}(4)$, J. Algebra, 13 (1969), 513-516.

28. J. Walter, The characterization of finite groups with abelian Sylow 2-subgroups, Ann. Math., 89 (1969), 405-514.

Received April 14, 1976 and in revised form December 14, 1976. This research was supported by NSF Grants, MPS 74-07807, MPS 71-03070, and MPS 75-07512.

UNIVERSITY OF CALIFORNIA

SANTA CRUZ, CA 95064 



\section{PACIFIC JOURNAL OF MATHEMATICS}

\section{EDITORS}

RICHARD ARENS (Managing Editor)

University of California

Los Angeles, CA 90024

Charles W. Curtis

University of Oregon

Eugene, OR 97403

C. C. MOORE

University of California

Berkeley, CA 94720

\section{J. DugundJI}

Department of Mathematics

University of Southern California

Los Angeles, CA 90007

R. FinN and J. Milgram

Stanford University

Stanford, CA 94305

ASSOCIATE EDITORS
E. F. BECKENBACH
B. H. NeumanN
F. WOLF
K. YOSHIDA

\section{SUPPORTING INSTITUTIONS}

UNIVERSITY OF BRITISH COLUMBIA CALIFORNIA INSTITUTE OF TECHNOLOGY

UNIVERSITY OF CALIFORNIA

MONTANA STATE UNIVERSITY

UNIVERSITY OF NEVADA, RENO

NEW MEXICO STATE UNIVERSITY

OREGON STATE UNIVERSITY

UNIVERSITY OF OREGON

OSAKA UNIVERSITY

\author{
UNIVERSITY OF SOUTHERN CALIFORNIA \\ STANFORD UNIVERSITY \\ UNIVERSITY OF HAWAII \\ UNIVERSITY OF TOKYO \\ UNIVERSITY OF UTAH \\ WASHINGTON STATE UNIVERSITY \\ UNIVERSITY OF WASHINGTON \\ AMERICAN MATHEMATICAL SOCIETY
}

The Supporting Institutions listed above contribute to the cost of publication of this Journal, but they are not owners or publishers and have no responsibility for its content or policies.

Mathematical papers intended for publication in the Pacific Jaurnal of Mathematics should be in typed form or offset-reproduced, (not dittoed), double spaced with large margins. Please do not use built up fractions in the text of your manuscript. You may however, use them in the displayed equations. Underline Greek letters in red, German in green, and script in blue. The first paragraph or two must be capable of being used separately as a synopsis of the entire paper. Items of the bibliography should not be cited there unless absolutely necessary, in which case they must be identified by author and Journal, rather than by item number. Manuscripts, in triplicate, may be sent to any one of the editors. Please classify according to the scheme of Math. Reviews, Index to Vol. 39. All other communications should be addressed to the managing editor, or Elaine Barth, University of California, Los Angeles, California, 90024.

The Pacific Journal of Mathematics expects the author's institution to pay page charges, and reserves the right to delay publication for nonpayment of charges in case of financial emergency.

100 reprints are provided free for each article, only if page charges have been substantially paid. Additional copies may be obtained at cost in multiples of 50 .

The Pacific Journal of Mathematics is issued monthly as of January 1966. Regular subscription rate: $\$ 7200$ a year (6 Vols., 12 issues). Special rate: $\$ 36.00$ a year to individual members of supporting institutions.

Subscriptions, orders for back numbers, and changes of address should be sent to Pacific Journal of Mathematics, 103 Highland Boulevard, Berkeley, California, 94708.

PUBLISHED BY PACIFIC JOURNAL OF MATHEMATICS, A NON-PROFIT CORPORATION

Printed at Kokusai Bunken Insatsusha (International Academic Printing Co., Ltd.). 8-8, 3-chome, Takadanobaba, Shinjuku-ku, Tokyo 160, Japan.

Copyright (C) 1975 by Pacific Journal of Mathematics Manufactured and first issued in Japan 


\section{Pacific Journal of Mathematics}

\section{Vol. 71, No. $2 \quad$ December, 1977}

Krishnaswami Alladi and Paul Erdős, On an additive arithmetic

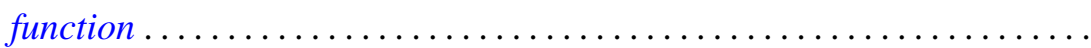

James Bailey and Dale Rolfsen, An unexpected surgery construction of a

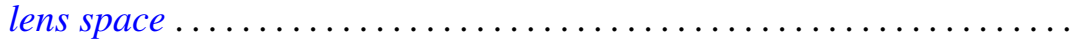

Lawrence James Brenton, On the Riemann-Roch equation for singular

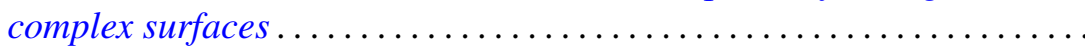

James Glenn Brookshear, Projective ideals in rings of continuous

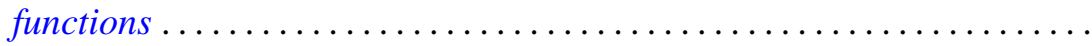

Lawrence Gerald Brown, Stable isomorphism of hereditary subalgebras of

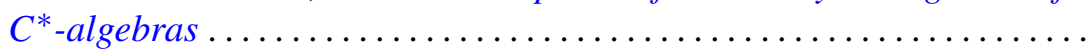

Lawrence Gerald Brown, Philip Palmer Green and Marc Aristide Rieffel, Stable isomorphism and strong Morita equivalence of $C^{*}$-algebras....

N. Burgoyne, Robert L. Griess, Jr. and Richard Lyons, Maximal subgroups

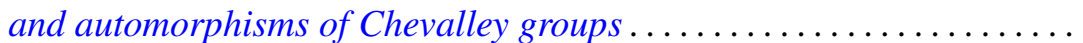
365

Yuen-Kwok Chan, Constructive foundations of potential theory .... 405

Peter Fletcher and William Lindgren, On $w \Delta$-spaces, $w \sigma$-spaces and

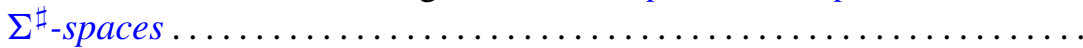

Louis M. Friedler and Dix Hayes Pettey, Inverse limits and mappings of

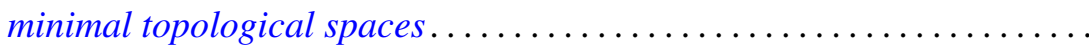

Robert E. Hartwig and Jiang Luh, A note on the group structure of unit regular ring elements.

I. Martin (Irving) Isaacs, Real representations of groups with a single involution ...

Nicolas P. Jewell, The existence of discontinuous module derivations . .

Antonio M. Lopez, The maximal right quotient semigroup of a strong semilattice of semigroups .......................

Dennis McGavran, $T^{n}$-actions on simply connected $(n+2)$-manifolds

Charles Anthony Micchelli and Allan Pinkus, Total positivity and the exact $n$-width of certain sets in $L^{1}$.

Barada K. Ray and Billy E. Rhoades, Fixed point-theorems for mappings with a contractive iterate .......................

Fred Richman and Elbert A. Walker, Ext in pre-Abelian categories. .

Raymond Craig Roan, Weak* generators of $H^{\infty}$ and $l^{1}$..

Saburou Saitoh, The exact Bergman kernel and the kernels of Szegö type...

Kung-Wei Yang, Operators invertible modulo the weakly compact 